\title{
A potential way to unify classical and quantum mechanics
}

\author{
*** \\ Author: Roberto Napolitano (born in Naples, Italy, on the $7^{\text {th }}$ of April, 1975) \\ https://orcid.org/0000-0002-0985-1982 \\ Contacts: LENNYUS@ALICE.IT \\ Independent researcher \\ $* * *$
}

Abstract

In the present work, by moving from the assumption that the Sun (and all the massive bodies) produces, starting from a certain distance from it, attractive and repulsive gravitational forces at the same time, giving life to the movement of the planets around the Sun according to the same principle of pendulum, I managed to derive a perihelion precession formula, a black hole radius formula and, above all, a formula of the atomic nuclear radius, which was missing until now, all in excellent agreement with the observation and in a completely independent way of the Einstein's theory of relativity.

I have also shown that the nuclear radius formula can also be successfully used to predict the radius of neutron stars.

Moreover I have found - always through the same principles that allowed me to achieve the above results, in particular through the modification of the Newtonian gravitational potential, in turn due to the different modus-operandi of gravity force - a formula of the non-decreasing orbital velocity of galactic stars, without considering dark matter.

Then I have demonstrated the black hole is composed only of protons, and that it's similar to the nucleus of the atom and, analogously, the galaxy is similar to the atom, since the stars moving around the central nucleus in the same way as the electrons move around the atomic nucleus.

I have also found another similitude among atomic nucleus, black hole and neutron stars, namely the self-orbiting phenomenon existing in all the cases.

From the mathematical findings obtained in the present work it has also emerged the existence, both at the microscopic and the macroscopic level, of the gravitoelectric force (or, if one prefers, electro-gravitational force), resulting from 
the fusion of the gravitational force with the electrostatic one, working exactly in accordance with Newtonian mechanics, although modified by the introduction of a repulsive force in addition and in opposition to the attractive one, that makes us understand the universe works always in the same way, both in macro and in micro.

Furthermore, by means of the theory here proposed, it has been possible to find a theoretical foundation to the Planck constant, to derive the photon mass, to derive the electron orbital radius, inner and outer, as well as to prove the existence of the gravitoelectric radiations.

It is also emerged the existence of the universal principle of specific asymmetry between gravitational potential energy and kinetic energy, as a cause of nuclear energy $E=m c^{2}$.

In this perspective, the present work can represent a potential unifying way between the macrocosm and microcosm mechanics. 


\section{CONTENTS}

$* * *$

\section{Chapter I}

\section{The pendulum in the universe}

1.1 The context.....................................................

1.2 Introduction...............................................

1.3 The non-summability between centripetal acceleration and gravity acceleration in the motion of the planets............................13

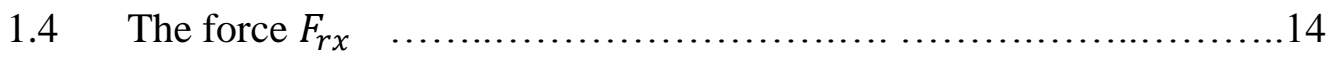

1.5 First analogy between solar system and pendulum.................. 17

1.6 Second analogy between solar system and pendulum .............. 17

1.7 Third analogy between solar system and pendulum .............. 17

1.8 Fourth analogy between solar system and pendulum ................ 19

1.9 Fifth analogy between solar system and pendulum.................20

1.10 Sixth analogy between solar system and pendulum................21

1.11 Seventh analogy between solar system and pendulum...............21

1.12 Strength analysis of the $F_{r x}$ force..............................21

1.13 The cause of the motion of the planets around the Sun and all the celestial bodies moving around a gravitational center ................22

1.14 The cause of the orbital ellipse...................................24

1.15 The gardener's ellipse.........................................29

1.16 Energy of the attractive-repulsive gravitational field.................. 31

1.17 Difference between field of inexistent forces and field of self-canceling out forces.................................................... 31

1.18 Aphelion as the point from which the motion of the planets has begun ..................................................... 32

1.19 The necessary ellipticity of the orbit of the celestial bodies around a central body .32

\section{Mathematical demonstrations}

2.1 Demonstration of Kepler's third law by hypothesizing that the solar system is a harmonic oscillator.... .33

2.2 Demonstration that the planetary system is a harmonic oscillator......34

2.3 The accelerated expansion of the universe explained by the exclusively 
repulsive effect of gravity

\section{Chapter III}

\section{The ligamentous nature of gravity}

3.1 Gravitational ligaments .43

3.2 Space-time network or gravitational ligaments network?

3.3 Gravitational waves and their compatibility with ligamentous nature of gravity force.

$$
* * *
$$

\section{Chapter IV}

4. Gravitational attractive and repulsive forces, centripetal force and centrifugal force: differences

4.1 Definition of centripetal force and of centrifugal force................48

4.2 Centripetal and centrifugal forces in the solar system...............50

$* * *$

\section{Chapter V}

5. Perihelion precession, black hole radius, atomic nuclear radius, radius of neutron star and stars orbital speed

5.1 Perihelion precession........................................57

5.2 Gravity force: exception to the first principle of dynamics...........62

5.3 The deflection of stars-light behind the Sun.......................64

5.4 The black hole radius......................................66

5.5 The nuclear radius: gravitoelectrostatic force......................69

5.6 The non-decreasing velocity of galaxy stars: dark matter crisis........73

5.7 Black holes: "proton stars".................................... 77

5.8 The orbital speed and the orbital radius of the electrons ...............78

5.9 Principle of specific asymmetry between gravitational potential energy and kinetic energy as an ideal condition of orbital stability ..........85

5.10 The radius of neutron stars: differences with respect to the proton stars .97

5.11 The characteristics of shadow particles connected to the nucleons ...................................................101

5.12 The self-orbiting-phenomenon: mathematical tests...................105

5.13 The reason of non-decreasing velocity of stars and of electrons......106

5.14 The velocity of nucleons..................................... 108

5.15 The gravitoelectrostatic force: differences with the electrostatic force 
and with the gravitational force. 108

5.16 The Heisenberg's uncertainty principle: ontological characteristic of microscopic particles, or human limit?

5.17 Gravitational entanglement.................................... 112

Conclusions

\section{Chapter I}

\section{The pendulum in the universe}

$* * *$

\section{- $\quad 1.1$ - The context}

Does gravity also work at microscopic level?

If yes, how?

These are the questions to which science tries to give an answer, which is long in coming.

Einstein's theory of relativity gives good results at macroscopic level (even if, at galactic level, it does not explain the speed of the stars which, unlike the KeplerianNewtonian system, remains almost constant, regardless of the distance of the star from the galactic center), but does not work at microscopic level, where experiments have shown that the principle of local realism, on which the theory of general relativity is based, does not apply to microscopic particles and their dynamics.

In essence, Einstein believes that the force of gravity is the consequence of the spacetime curvature, caused by the mass of the bodies.

The force of gravity, in other words, would not be a force that acts instantaneously between the bodies, but acts at the speed of light through the deformation of the spacetime caused by the mass of the bodies.

The main difference between the theory of relativity and Newton's theory is that the latter believed that the force of gravity is immediately acting at distance, unlike the German scientist who believed that it travels at the speed of light thanks to a mediator: the space-time.

In truth, the non-applicability of relativity to the microcosm is not the only issue connected to this theory.

As anticipated above, relativity is not perfectly applicable even at galactic 
level, unless we resort to the existence of the hypothetical dark matter, which is believed to form about $75 \%$ of the universe, despite being completely invisible and untraceable.

So current theories try to find the physical and mathematical principles that unite the macroscopic and the microscopic world.

All the current theories are based on Einstein's relativity, in particular they suppose the existence of spacetime, even if they attribute different properties to it.

According to the theory of particles (supergravity and string theory) spacetime would be flat (unlike general relativity), and in any case it would not be the mediator of gravity, which would be mediated by a virtual particle, called graviton, but it has never been found.

Actually not only the graviton has never been found, but WIMPs in general have never been found (i.e. the weakly interacting massive particles), which, among other things, would constitute dark matter.

Many experiments have been conducted from 2005 to date in order to detect dark matter, both in space and in laboratories, but without good results.

The best results obtained by particle theories concern the behavior of particles in accelerators, where the subatomic particles are collided to verify their comportment.

However, even if the theory in question is suitable to predict the behavior of the particles after having them collided inside the accelerators, it cannot find a satisfactory explanation of the behavior of particles inside their natural site, namely the atom, and in particular the nucleus of atom, where many questions remain open (first of all the nuclear radius).

Moreover, the mathematical results of the string theory are subjected to the renormalization procedure - that, among other things, works better by assuming the existence of a supersimmetric particle (i.e. a partner of the elementary particle endowed with a greater mass) - to adapt them to the microscopic scales, but this mathematical procedure is not accepted by everyone.

In contrast to the theory of particles there is the theory of loop gravity.

This theory tries to quantize the spacetime, and to demonstrate that a supposed feature of the microscopic world (namely the fact that he energy is discrete, rather 
than continue) can be extended also to the macroscopic scales.

The main criticism against the theory of loop gravity relates the fact that it fails in finding a convincing way of quantizing spacetime.

In the present work a new vision of the gravitational phenomenon will be exposed, completely different from the two theories mentioned above, which perhaps has some element in common more with the theory of supergravity, rather than with loop gravity, ending up demonstrating the existence of a symmetrical particle (not supersimmetrical, by having the same mass as the real partner) of the nucleons.

\section{- 1.2 - Introduction}

Galileo, one of the greatest scientists of all time, was nothing short of fascinated by the pendulum's motion, studying it to the point of dissecting every its little secret.

He realized, among other things, that the amplitude of the oscillation in the ascending phase is slightly less than the amplitude of the oscillation in the descending phase, from which he deduced that the pendulum, if there was no friction with the air, would move forever.

Nevertheless he did not see any analogy with the motion of the planets, which is also eternal.

But let's come to us.

It's known that the pendulum is a system formed by a fixed point called "fulcrum", to which a wire is tied, to whose opposite end a sphere is fixed so that it cannot fall to Earth in consequence of gravity force.

The trajectory described by the pendulum is perfectly circular or, better, it describes an arc of a circumference more or less large depending on the amplitude of the initial oscillation.

So I wondered what would happen if the external gravitational attraction came not from one side only, below the pendulum, but surrounded it at 360 degrees.

I answered this question by basing more on logical than on real experiments, also because it is not easy to recreate the conditions of the example above, and the answer was that the pendulum should make a complete revolution around the fulcrum and, if there were not the friction with the air, the revolution should be repeated 
forever, exactly as it happens in the motion of the planets.

But how could the external attraction surround the pendulum $360^{\circ}$ ?

Simple, imagining that the fulcrum exerts on the sphere a force both attractive and repulsive, at the same time.

This consideration allowed me to grasp a strong analogy between pendulum and solar system.

I therefore hypothesized that the solar system constituted a pendulum, to be precise a particular type of pendulum, in which the external gravitational attraction comes not only from one side, like in the classical pendulum, but surrounds it $360^{\circ}$.

More precisely, the cosmic pendulum would be so composed: the fulcrum of the pendulum would be the Sun; the sphere, or rather the spheres hanging from the different threads, would be the planets; the gravity force exerted by the Earth on the sphere of the pendulum would be replaced, in the solar system, by a repulsive force exerted on the planets by the Sun itself.

I hypothesized, in particular, that the Sun (but also all other celestial bodies, including the planets) exerted, beyond a certain distance from it, not only an attractive force, but also a repulsive force, of equal magnitude compared to the first.

In other words, every massive body would give rise, starting from a certain distance from it, not to a single pair of opposing forces, which would cancel each other out, but to an attractive-repulsive force field that would invade the space surrounding the body itself, and which would create the conditions for the birth of a pendulum, as will be better seen later.

Before analyzing the particular type of pendulum I have imagined to exist in the solar system, let's analyze in the next page the forces-field that we have in a particular type of pendulum called "harmonic oscillator", characterized by the fact that the amplitude of the oscillation is very small, first studied by Galileo.

Figure 1 - Pendulum harmonic-oscillator (very small oscillations) 


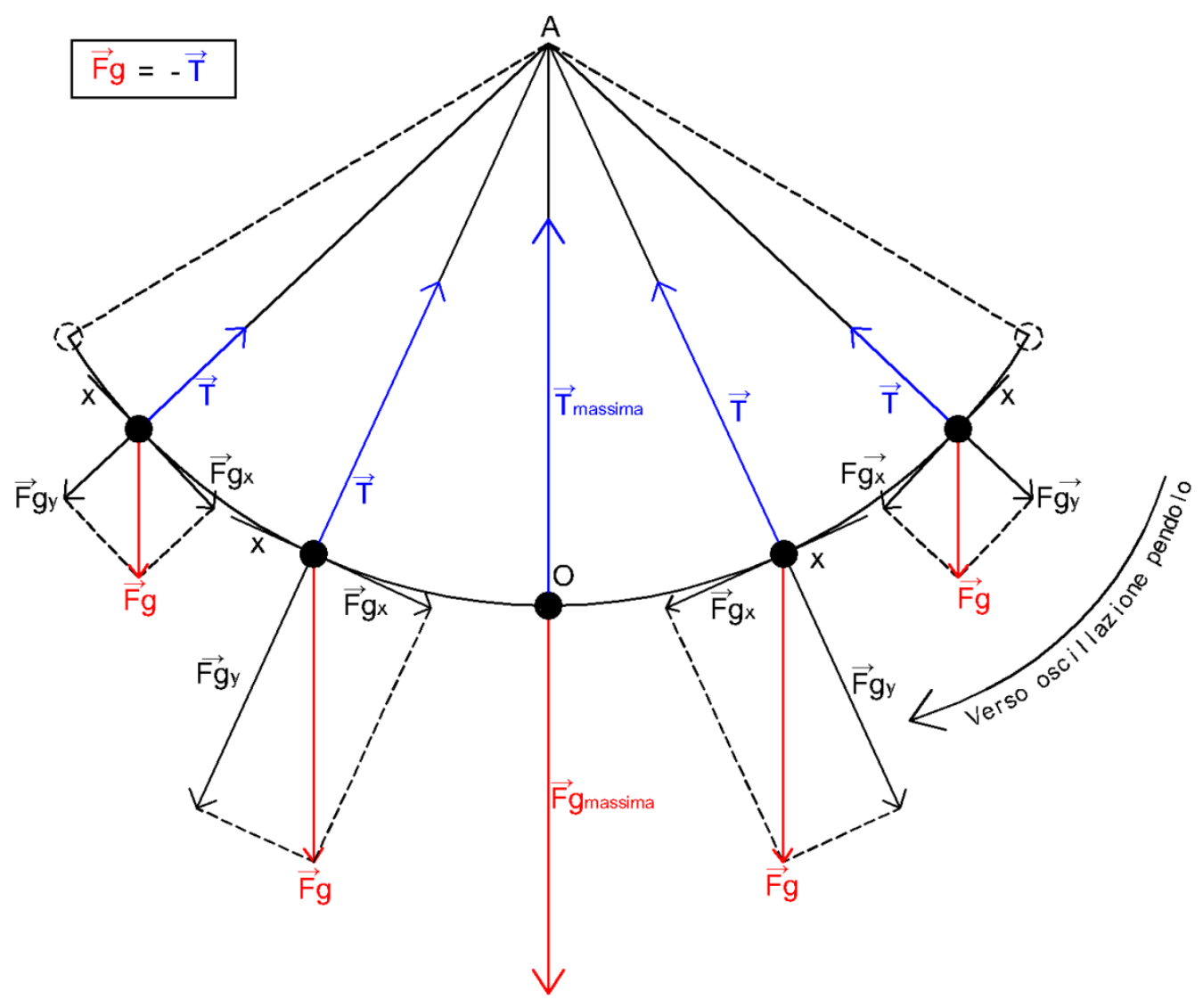

We note in the figure that in the Galilean pendulum there is only one point of equilibrium, the point $\mathrm{O}$, around which the sphere oscillates.

The sphere is always subjected to two forces: the gravity force $\left(F_{g}\right.$ indicated in red) and the reaction force $T$ (indicated in blue) that acts on the sphere since it is anchored at the fulcrum by the wire.

The force $F_{g}$ splits into two forces:

1) the force $F_{g y}$ : a component of gravity with a radial orientation (extension of the radius).

2) The force $F_{g x}$ : a component of the gravity force with orientation tangent to the trajectory of the pendulum. This force is responsible for the movement of the pendulum and exists because the gravity force and the tension of the thread are not aligned.

The force $T$ consists in a reaction force arising by the fulcrum, to which the sphere is fixed by the wire.

In other words, the sphere, by being bound to the fulcrum by the wire, reacts to 
the force $F_{g}$ with a force $T$ perfectly identical to the first.

This perfect identity between the two forces operates continuously during the oscillations of the pendulum, therefore, by continuously variating of the force $F_{g}$, the force $T$ varies continuously and to the same extent.

Therefore, in the descending phase, the force $F_{g}$ increases progressively as the sphere approaches the ground and, as it increases, the $T$ force also increases at the same time of an equal measure.

Moreover, the increase in speed is due precisely to the increase in gravity force that operates on the sphere in the descending phase.

In the ascending phase, then, the force $F_{g}$ decreases progressively as the pendulum moves away from the ground and, to this decrease of $F_{g}$, a simultaneous decrease in the same amount of force $T$ corresponds.

The decrease in speed is due to the fact that the sphere moves in the opposite sense to the gravity sense.

As I will show shortly, the solar system too constitutes a set of harmonic oscillator pendulums all having fulcrum in the Sun, in which the planets - but the principle can be extended to all the bodies of the universe moving around a gravitational center - are subjected to the same two forces to which the sphere of the harmonic oscillator pendulum is subjected, in particular, to a central attractive force coming from the Sun, and, at same time, to a repulsive one that pushes the planet outwards of the orbit, of equal-magnitude to the first, arising always from the sun.

The figure below shows the hypothetical diagram of the forces that should be realized if the solar system were a pendulum, thus assuming that the phase of approach to perihelion were identical to the descending phase of the pendulum, and that the phase of approach to aphelion were identical to the ascending phase of the pendulum, with the only difference that the motion is not oscillatory, but always has the same orientation (counterclockwise). 
Figure n. 2 - Cosmic pendulum harmonic oscillator

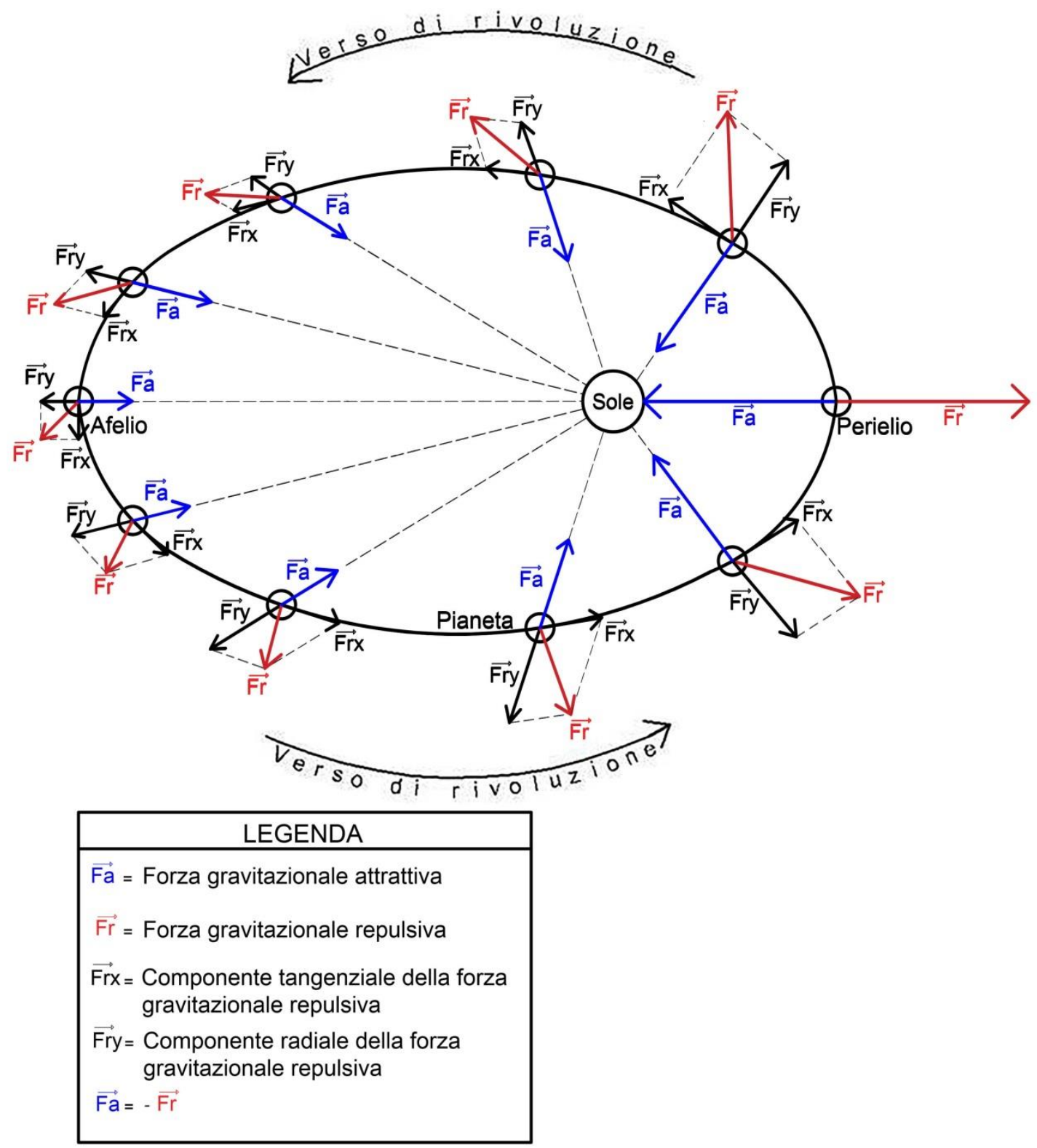

As we can see from the previous figure, the only point where the attractive and repulsive forces cancel each other out, by being exactly opposite, is perihelion: this happens because, as we will see better later, the planet, from the point following the perihelion, begins to move away from the Sun, and this can happen only if, in the instant in which the planet occupies the perihelion, it is subjected to two forces that cancel each other out, in which case, for the first principle of dynamics, the body moves, even if only for a moment, of rectilinear motion, so that it will cease to 
approach, curving, the Sun, and will begin to move away from it as little as possible.

When, instead, the planet is in aphelion, the two forces do not cancel out, because otherwise the planet would continue to move away from the Sun, but this would violate the principle of conservation of energy, because the attractive force would be suppressed, as we'll see better later, so that, in that point, the repulsive force cannot have radial orientation and to cancel itself with the attractive force.

In this way, in the planetary orbits the same conditions of the pendulum are replicated, in which, as seen in the figure 1 on page 9 , there is only one point where the two forces (the Earth's gravity and the tension of the wire) cancel each other out, that is the point closest to the Earth, defined "equilibrium point", where the magnitude of the conflict between them is maximum.

This equilibrium point of harmonic oscillator, therefore, is exactly equivalent to the perihelion of the cosmic pendulum, although the latter cannot properly be defined an "equilibrium point", because, if so, the planet would oscillate around it, but, as it is known, this does not happen.

The fact that it is not an equilibrium point in the proper sense of the term also has another fundamental implication: if the planet, for any reason (more theoretical than practical) stopped in perihelion, it, once removed the hypothetical obstacle, would not remain at rest like the sphere of the harmonic oscillator would do, but would resume to revolve around the Sun, even though not describing the same orbit as before, but an orbit characterized by the fact that the stopping point, which was assumed to be the perihelion, the new aphelion would become, with the consequence that the new perihelion would place in the exact opposite side to that in which it was before the stop.

This happens because, as will become clearer later, the aphelion is the point from which the motion of celestial bodies (subjected to an attractive-repulsive gravitational field) begins, similarly to the pendulum, in which the movement of the sphere starts from the point of the trajectory in where the gravity force is less.

I hypothesized that the attractive gravity force was always counterbalanced by the repulsive force, because, otherwise, the planet would sooner or later end up colliding against the Sun, due to the gravitational attraction exerted by the latter, which, in the absence of repulsive force, should inevitably wear out, with its incessant 
work, the planet-sun distance.

The only movement of the body with tangential orientation, in fact, cannot be considered sufficient to counteract gravity force, because this is an action force, not a reaction one, as we will better see later.

So I assumed that the force $T$, which in the classical pendulum exerts on the sphere a reaction force, was replaced, in the cosmic pendulum, by the attractive gravity force coming from the Sun, which I called $F_{a}$ (attractive force), and that the force $F_{g}$, which in the classical pendulum exerts an external attraction, was replaced, in the cosmic pendulum, by the repulsive gravity force always coming from the Sun, which I called $F_{r}$ (repulsive force).

I basically hypothesized that the following equation always occurred in the solar system:

$$
F_{r}=-F_{a}
$$

Where $F_{a}$ is the attractive gravitational force and $F_{r}$ is the repulsive gravity force.

The minus sign before $F_{a}$ indicates that the two forces have opposite orientations.

Consequently I hypothesized that, in the solar system, the attractive gravity acceleration, which we will call $g_{a}$ is always equal to the repulsive gravity acceleration, which we will call $g_{r}$, so we would have the following equation:

$$
g_{r}=-g_{a}
$$

- 1.3 The non-summability between centripetal acceleration and gravity

\section{acceleration in the motion of the planets}

It's important to clarify that it has been possible to hypothesize in (2) the equality between the two gravity accelerations (the attractive and the repulsive), since, although the planets are subjected to the centripetal force, it's not possible to sum the centripetal acceleration with the gravity acceleration (except in the perihelion, as we can see later).

The reason for this foreclosure is that the solar gravity force, in while attracting 
the planets towards the center, indirectly prevents them from moving away in a straight line and, in this way, behaves "also" like a constraint generating centripetal force.

The centripetal force, in fact, can be defined as the force that prevents from the distancing of the body in straight line from the center, making it move around it, so that if the central force is attractive, like gravity, it also acts as a constraint, because who attracts a body also prevents from its distancing from it (as if it were a rope to which the planet is hooked), but the gravity force is not only a constraint, by also generating an attraction on the body that tends to bring it closer to the attractor body.

Since the centripetal acceleration being the speed with which the body changes the orientation of its tangential velocity vector in a given time interval, we can say that, when the central force is attractive, like the gravitational one, centripetal acceleration can be considered an indirect effect of the central force and, therefore, centripetal acceleration and gravity acceleration cannot be summed algebraically, by not being generated by two different forces, but by being two distinct effects (the attractive-direct one and the constraining-indirect one) of a single force (the gravitational attractive force).

But the statement that they are two distinct effects of the same force is very important because by virtue of this statement one can sum the two accelerations (gravity acceleration and centripetal one) when they have the same orientation and sense, i.e. the same effect, like it happens in the perihelion, as we'll see later.

\section{- 1.4 - The force $F_{r x}$}

At this point a question arises: what does force $\boldsymbol{F}_{\boldsymbol{r} \boldsymbol{x}}$ derive from?

The question arises because, in the classical pendulum, its homologous force $F_{g x}$ derives from the fact that the gravity force doesn't have the same orientation as the reaction force $T$, so it splits into two forces, one of which is $F_{g x}$.

In the cosmic pendulum, vice versa, the repulsive force should have the same orientation as the attractive one, therefore, strictly speaking, the force $F_{r x}$ should not arise.

The only possible explanation of its arising is to believe that this force arises 
from the fact that the Sun creates a 360-degree attracting-repulsive field, thus determining the arising not of a single pair of opposing forces operating on the planet (an attractive and a repulsive one) that should cancel each other out, with the consequent quiet state of the planet, but an infinite number of pairs of equal and divergent forces, with consequent different levels of energy (or pressure) resulting from their contrast, and this would cause the revolution of planet around the Sun according to the principle illustrated in the paragraph 1.13 .

The figure in the next page will help us to better understand the peculiar phenomenon of the pendulum subjected to a 360-degree attraction-repulsive action by the fulcrum.

Figure n. 3

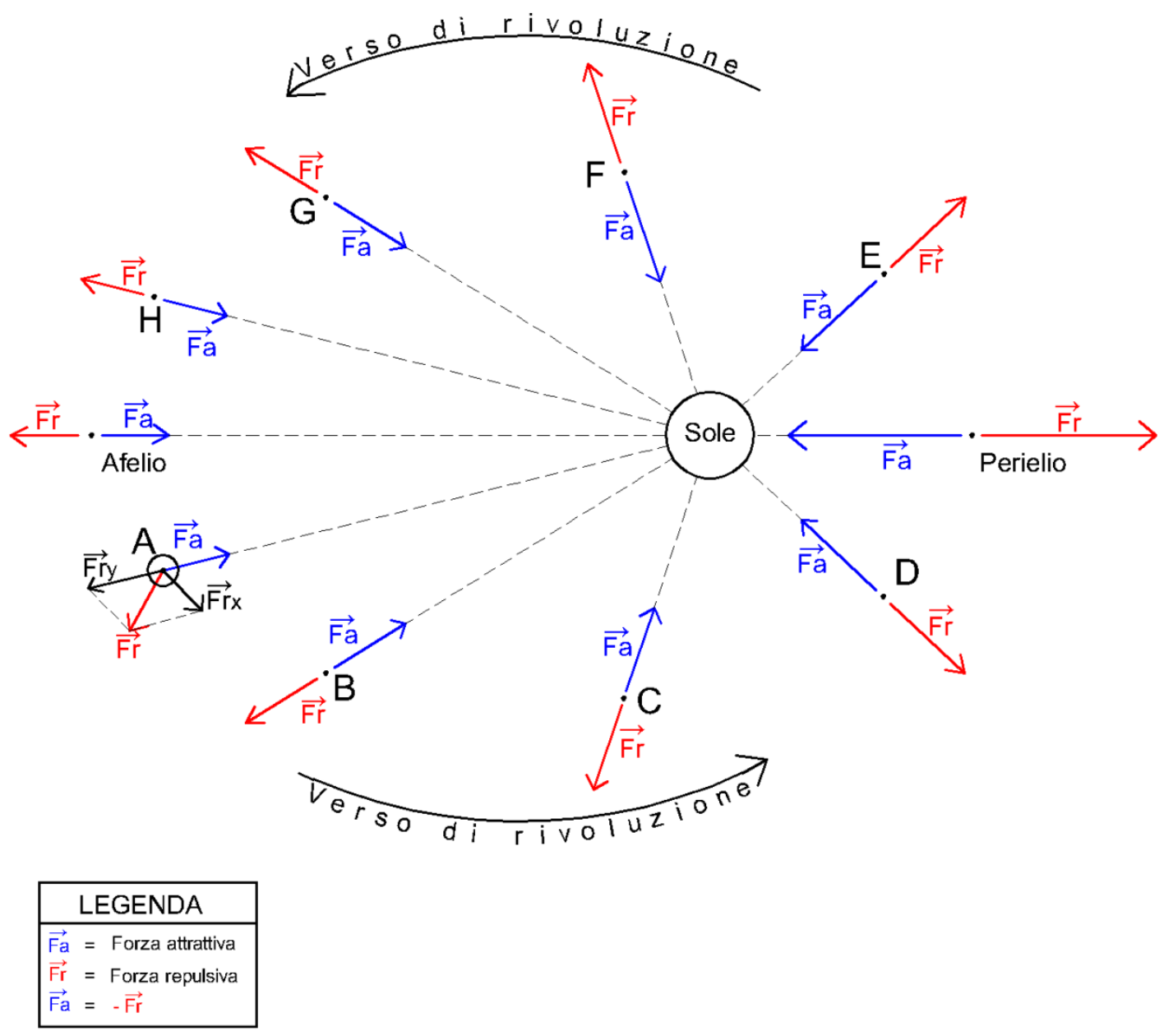

It's important to understand that the birth of force $F_{r x}$, and therefore the 
composition of the two forces $F_{r x}$ and $F_{r y}$ in $F_{r}$, occurs only when the planet occupies a new point in the orbit.

Before that moment the two forces present in each points of the attractiverepulsive field cancel each other out from the dynamic point of view, because they have the same magnitude, the same orientation and opposite sense, but their collision generates energy (or pressure, it's not very clear to me), and this energy has different values depending on the point considered: closer to the Sun the energy would be greater than that existing in the points further from the star.

When the planet moves to the next point in the orbit, the $F_{r}$ force, due to the birth of the $F_{r x}$ force, will result from the composition of the two forces $F_{r x}$ and $F_{r y}$, and in this way it will no longer have the same orientation as the attractive force $F_{a}$, and this creates the same type of forces-field that exists in the harmonic oscillator pendulum, in particular two equal but not opposite forces (not aligned), which in turn determines the movement of the planet around the Sun according to the principle illustrated in the paragraph 1.13 .

This is my guess, by having never been observed in nature the behavior of a body inserted in an attractive-repulsive forces-field generated by a single central body, but I think that my deduction is the only one able to explain the cause of the movement of the celestial bodies around a central body, which otherwise would have to be ascribed to a mysterious thrust, or rather to multiple thrusts (by having every single planet a different velocity with respect to the other ones), which, to tell the truth, is rather far-fetched.

In this regard, we can observe that any known field of forces (magnetic, electromagnetic, electric) never exerts two opposing forces at the same time, and never in the same region of space.

In the magnetic field, for example, the magnetic charges are arranged in a polar fashion, i.e. one type of charge is concentrated in one pole of the magnet, and the opposite charge is concentrated in the opposite side of the magnet, so that the space surrounding the magnet can be divided in two areas: one pervaded by a positive charge, and the other pervaded by a negative charge.

In the electric field, furthermore, the opposite charges never derive from the 
same body (for example the electrostatic attractive-repulsive field generated by two neighboring wires, in which case one wire is crossed by positive electric current, and the other by negative electric current).

Therefore, in reconstructing the behavior of a body inserted in an attractiverepulsive gravitational field, I have had to proceed necessarily to the formulation of hypotheses and, among all, the most likely has seemed to me that such a kind of forces-field should give rise to the birth of a pendulum which does not oscillate, but revolves around the fulcrum.

The alternative would be to assume that the body inserted in such a kind of forces-field would remain in a state of rest, between two opposing forces, but this would seem contradicted by the fact that, as we'll see shortly, the planets move with the same orbital period, with the same angular velocity and above all with the same acceleration law of the Galilean harmonic oscillator, which suggests that the conditions for the birth of a pendulum exist with high probability, i.e. the presence of two conflicting forces on the planet, even if not "opposite".

Moreover, the analogies with the pendulum of Galileo are not limited to the applicability to the planetary motion of the aforementioned mathematical laws regulating the motion of the harmonic oscillator, since also other physical analogies subsist, set forth below.

\section{Physical analogies between solar system and pendulum}

\section{- 1.5 - First analogy}

The sphere of the pendulum oscillates around a fixed point, called the fulcrum, just like the planets move around a fixed point, the Sun.

\section{- $\quad 1.6$ - Second analogy}

The motion of the planets is perpetual, as perpetual would be the one of pendulum in the absence of friction with the air and if the wire was perfectly inextensible.

\section{- 1.7 - $\underline{\text { Third analogy }}$}

The planets move in not uniformly accelerated way when they move towards 
the attractor.

More precisely, the planets accelerate not uniformly when they move towards perihelion (point of the orbit closest to the Sun and of maximum orbital velocity), analogously to the pendulum, which accelerates not uniformly in the descending phase, reaching the maximum linear velocity in the closest point to the Earth.

When the planet is directed towards the perihelion, it progressively approaches the Sun, though not directly, so the magnitude of the conflict between $F_{a}$ and $F_{r}$ increases, as seen in figure n. 2.

The progressive increase of the two opposing forces also determines the progressive increase of the planet's orbital velocity, up to the maximum velocity in the perihelion.

The increase in the speed of the planet is due to the fact that the increase in the magnitude of the divergent forces operating on the planet causes the force $F_{r x}$ to increase too.

The force $F_{r x}$ in fact, can only be born if we hypothesize the simultaneous reduction of the force $F_{r}$ that pushes the planet outwards, otherwise a force would be created from nothing, which would violate the energy conservation law.

Therefore, the greater the force $F_{r}$, the greater will be the force $F_{r x}$, thanks to which the planet will move towards the nearby point of the orbit that, from the energetic point of view, is the least strong between the strongest ones, or the least weak among the weakest ones, as will be seen shortly.

The phase of approach to perihelion is therefore similar to the descending phase of the Galilean pendulum, where the sphere, while accelerating progressively as it approaches the surface of the Earth, is subjected to two divergent and equal forces, though not "opposite", which gradually increase in magnitude: the analogy between the two phenomena is confirmed by the fact that, in both cases, the acceleration is not uniform.

The perihelion is similar, therefore, to the equilibrium point of the Galilean harmonic oscillator, but this similarity is limited to the fact that in both cases the two opposing forces cancel each other out from dynamically view-point, but, as mentioned above, the perihelion has absolutely not to be considered like an equilibrium point, 
because, if by hypothesis the planet would stop there, it would not remain motionless like would happen if the harmonic oscillator stopped in the equilibrium point, but it would re-begin to revolve around the attractor-repulsor with a new orbit having the aphelion in the old perihelion.

\section{- 1.8 - Fourth analogy}

The planets decelerate unevenly when they move towards the aphelion (point farthest from the Sun and of minimum orbital velocity), similar to the pendulum, which decelerates not uniformly in the ascending phase, reaching the minimum tangential velocity at the farthest point from the Earth.

When the planet moves away from the perihelion and goes towards the aphelion, the magnitude of the collision between the attractive force and the repulsive force decreases progressively, until it touches the minimum in aphelion, and this determines the reduction of the force $F_{r x}$ and, therefore, the slowing down of the planet: the analogy between the two phenomena is confirmed by the fact that, in both cases, the deceleration is not uniform.

In this way, the same conditions existing in the classical pendulum are replicated in the solar system: an external gravitational force that pushes the body away from the center continuously and adequately countered by a force pointing towards the central point, not opposed to the first one.

It's important to clarify that during this phase the planet slows down because the orientation of attractive gravity force is opposed to the orientation of planet's velocity, by been not orthogonal to the motion, so this force hinders the planet by making it slow down, but not enough to make the orbit be parabolic, since it is avoided from the fundamental contribution of the tangential component of the repulsive force $\left(F_{r x}\right)$, which allows to preserve the ellipticity of the orbit, and therefore its stability over time.

As it has been said before, the aphelion, point of the orbit in which gravity is less, is the one from which the motion of celestial bodies begins in a repulsiveattractive forces-field, similarly to what happens in the pendulum, in which the motion of sphere begins from the point where the gravity force is less strong. 


\section{Mathematical analogies between solar system and pendulum}

\section{- 1.9 - Fifth analogy}

As for mathematical analogies, first of all we have that the orbital period of the planets, i.e. the time in which the planets make a complete revolution around the Sun, depends, as I will show later, on the repulsive gravity force and on the distance from the Sun, more precisely the revolution time of the planet is expressed by the following formula:

$$
T=2 \pi \sqrt{\frac{L}{g}}
$$

that is exactly the law regulating the period of the harmonic oscillator discovered by Galileo, where " $L$ ", in the pendulum of Galileo, is the length of the wire to which the sphere hangs, and " $g$ " is the gravity acceleration exerted on the sphere by the Earth (or by the different planet on whose surface the pendulum is possibly placed), instead, in the "cosmic pendulum" (that is what I saw in the solar system), " $L$ " becomes the average distance between planet and Sun (i.e. the semi-major axis $" a ")$, and " $g$ " becomes the average repulsive gravity acceleration due to the solar repulsive force.

We point out that the formula written above is the one regulating the period of a particular type of pendulum called "harmonic oscillator", in which, as already mentioned, the amplitude of the oscillation is so small that it does not allow the centripetal force to rise if not to a negligible extent, due to the minimum speed of the sphere and to the relevant length of the radius, so that here too, like in the cosmic pendulum, there is a continuous equality between the central force and the external force.

Thus, in the cosmic pendulum, the repulsive gravity force, which we have indicated above with $F_{r}$, has the same function as the earth's gravity force of the harmonic oscillator of Galileo, i.e. to push the body away from the center, and the attractive gravity force has the same function as the centripetal force in the Galilean pendulum.

The analogy between the two phenomena (pendulum harmonic oscillator and solar system pendulum) allows us to apply to the solar system the laws regulating the 
period, the angular velocity and acceleration of pendulum harmonic oscillator.

The only difference between the two pendulums is that the Galilean one oscillates from one side to the other around the equilibrium point, instead the cosmic one makes a complete revolution around the center, but this is due to the continuous change of orientation of the repulsive gravity, as seen in figure n. 2 , and to the fact that in it there is no real equilibrium point, as stated above.

\section{- 1.10 - Sixth analogy}

The angular velocity of the planets orbiting around the Sun is equal to the angular velocity of harmonic oscillator.

The angular velocity of the harmonic oscillator, as is known, is:

$$
\omega=\sqrt{\frac{g}{L}}
$$

As will be shown below, the planetary angular velocity formula is exactly the same as pendulum formula written above, so we will have:

$$
\omega=\sqrt{\frac{g}{a}}
$$

where $a$ is the semi-major axis.

\section{- 1.11 - Seventh analogy}

The acceleration law of the planetary revolution motion is equal to the acceleration law of the harmonic oscillator.

It's known that the acceleration law of the Galilean harmonic oscillator is:

$$
g=\omega^{2} x
$$

where $x$ is the position of the body, i.e. its projection on the abscissas axis.

As we will show later, this is also the acceleration law regulating the revolution motion of the planets around the Sun.

\section{- $\quad 1.12$ - Strength analysis of the $F_{r x}$ force}

As already mentioned, but the discourse deserves further study, it does not come from nothing, but comes from the reduction of $F_{r}$. 
More precisely, when the planet moves from one point to another in its orbit, it happens that the force $F_{r}$ - which before the advent of the planet in that point was perfectly opposite and equal to $F_{a}$ - reduces itself by an amount sufficient to allow the arising of a force that makes the planet move to the next point of the orbit, respecting the two principles enunciated in the next pages.

Since the force $F_{r x}$ derives from the reduction of force $F_{r}$, it follows that, on the one hand, the principle of conservation of energy is not violated (nothing is created or destroyed, but everything is transformed or yielded) and, on the other hand, makes the planet move within the attractive-repulsive field.

To recapitulate, $F_{r}$ is reduced into $F_{r y}$ and the sur-plus of energy deriving from this reduction gives life to the force $F_{r x}$.

The resultant between $F_{r x}$ and $F_{r y}$ will be $F_{r}$, but, due to the composition of these two forces, the $F_{r}$ force will be no longer aligned with $F_{a}$ (unlike when that particular point of the orbit is not yet occupied by the planet); in other words they will be no longer "opposite", so they will no longer nullify themselves, and this will cause the curved trajectory of the planet, according to the same principle that regulates the pendulum.

This physical mechanism is a peculiarity of a particular type of pendulum, in which the center exerts both attractive and repulsive force on the sphere, at 360 degrees, and the evidence that allows to support the existence of this particular physical phenomenon would be, in my opinion, not only the physical and mathematical analogies between the pendulum and the solar system seen above, but also the ellipticity of planetary orbits.

Let's see why.

- 1.13 - The cause of the motion of the planets around the Sun and all the celestial bodies moving around a gravitational center

The big-bang has probably given rise to the masses of celestial bodies, but not also to their movement in space.

The movement would be due, in my opinion, to the existence of an attractive- 
repulsive forces-field generated by the central massive body, starting from a certain distance from it, directly proportional to the mass: the greater the mass, the greater will be the distance from which the repulsive force would come into existence in addition to the attractive one.

I hypothesized, in particular, that a body inserted in an attractive-repulsive field would not remain stationary due to the cancellation of two opposing forces (as Newton teaches), as in a first analysis it might be thought, but would move around the center, because the body, despite of being subjected to two opposing forces, would feel the presence around itself of different energy (or pressure) values because of the different magnitude of the collision between the attractive and repulsive forces, depending on the distance from the central body (the gravity force is inversely proportional to the square of the distance, and this also applies to repulsive force).

In truth, this just described would not be, in my opinion, the only case of an attractive-repulsive force-field, but probably there exists also another one in nature: i.e. the one formed around the nucleus of the atom, which causes the perpetual movement of the electrons around it, as we will see better at the end of this paper.

The clash between the two forces creates, therefore, the conditions for the birth of the pendulum.

The only difference between the Galilean pendulum and the cosmic pendulum is that, as we have already said, in the first case the gravity force comes only from one side, so that the pendulum does not rotate, but oscillates around the equilibrium point, instead in the cosmic pendulum the orientation of the repulsive force changes continuously, and a real equilibrium point is missing, so that the oscillation turns into rotation around the fulcrum, and moreover in the Galilean pendulum the central force is a reaction force, instead in the cosmic pendulum it is an action force, having an attractive nature.

Each point of the orbit, therefore, is never equivalent, from the view-point of the energy (or pressure) value, to the previous one or to the next one, because it will have a greater or lesser energy depending on whether, respectively, it is closer or farther from the central body with respect to the point where the planet 
is located, and the latter, as will be seen, tends to move, among the points with stronger energy existing in its surroundings, towards the one with less strong energy, or, among those with weaker energy, towards the one with less weak energy, depending on whether, respectively, it is in the approaching-phase or distancing-phase from the Sun.

\section{- $\quad 1.14$ - The cause of the orbital ellipse}

The movement of a body immersed in an attractive-repulsive forces-field is governed by the principle described in the following pages.

Before explaining the principle it is necessary to redo figure n. 3 , seen in the paragraph 1.3.

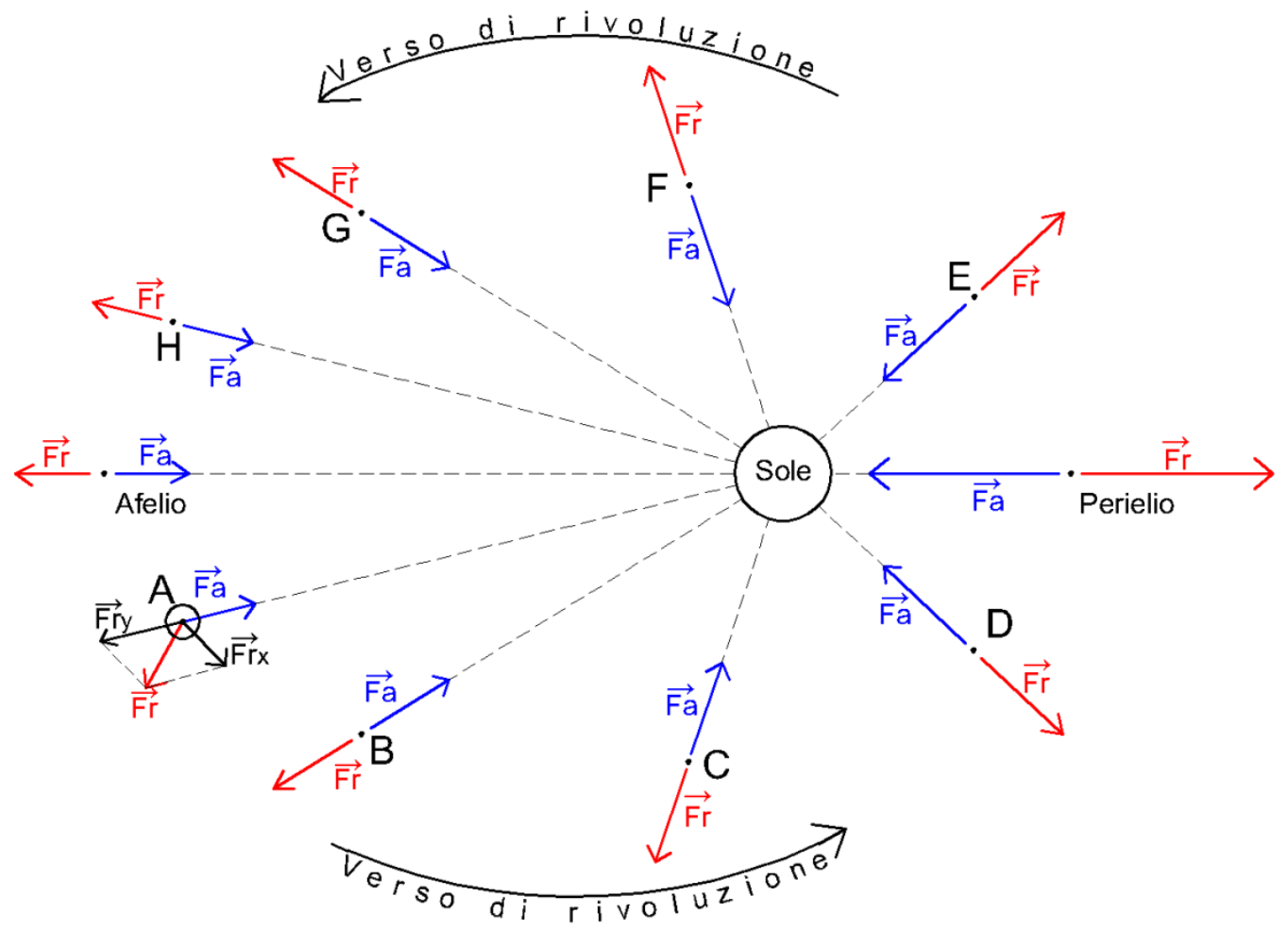

\footnotetext{
LEGENDA

$\overrightarrow{\mathrm{Fa}}=$ Forza attrattiva

$\overrightarrow{\mathrm{Fr}}=$ Forza repulsiva

$\overrightarrow{\mathrm{Fa}}=-\overrightarrow{\mathrm{Fr}}$
}

Principle of minor possible approach and minor possible distancing from the attractor-repulsor

If we assume that a body inserted in an attractive-repulsive gravitational field 
does not remain in a state of rest, but moves, it is reasonable to suppose that it moves in such a way to approach to the attractor-repulsor as little as possible and, subsequently, moving away as little as possible from it, cyclically.

We can assume, in particular, that the clash between the two opposing forces generates a certain value of energy (or pressure), and that this energy would be decreasing in relation to the square of the distance from the central body (since the magnitude of the gravitational force decreases according to this ratio also with regard to the repulsive force), therefore, in such a non-uniform energetic context, it seems more sensible to assume that the body moves from one energy level to another, continuously, rather than hypothesizing that it moves in a circular way around the attractor-repulsor, at constant distance from it, in which case the body would move senselessly always occupying the same level of energy (or pressure).

It is reasonable to assume, therefore, that in the first phase the body approaches to the central one, and that in the following phase it gets away from it, followed again by the approaching phase and so on, in a cyclical succession of the two phases.

During the approaching phase to the attractor-repulsor, the body is pushed, among all the points having a greater energetic value existing in its surroundings, towards the less strong one.

In geometrical terms, the planet, while it is approaching the Sun, is pushed, among all the points closest to the Sun existing in its surroundings, towards the less near point.

By following this principle, the planet approaches the central body as little as possible, i.e. in the most gradual manner possible.

As shown in the figure on the previous page, the planet, from point $\mathrm{A}$, moves to B (only for clarity reasons, point B has been drawn slightly moved by A, but it should be imagined as contiguous to it): this point, compared to A, approaches the Sun as little as possible.

By following the same criterion, the planet will move from point $B$ to point $C$, and so on, until the perihelion is reached, as gradually as possible, in such a way not to violate the principle of energy conservation, which would be injured if the body approached the attractor-repulsor in an insufficiently gradual manner, thus suppressing the repulsive force. 
But how can a body A approaches to a body B as little as possible?

The only way is to move A on an elliptical trajectory in which B is in one of the foci, and the second focus is as close as possible to B.

In this way, A will travel along a trajectory characterized by the fact that, while moving towards B, it moves away, at the same time, as little as possible from a point very close to B (the other focus), since, as will be seen below, "the ellipse is the geometric place of the points of the plane where every point, compared to the adjacent one, approaches as little as possible a focus and at the same time moves away as little as possible from the other focus ": in this way A will approach B as little as possible, since, while it does so, it moves away at the same time as little as possible from a point (the other focus) which is very close to $B$.

The principle of the "minor possible approach" to the attractor-repulsor, however, is not applicable throughout the planet's orbit.

It is reasonable to suppose, in fact, that in an attractive-repulsive forces-field generated by a central body, the existence of the two opposing forces causes the minor possible approach to take place only up to a certain limit, beyond which the further approach to the attractor-repulsor would violate the principle of energy conservation, by suppressing the repulsive force.

The point of planetary orbit where this particular limit situation would occur is, in my opinion, the perihelion, beyond which, if the body continued to approach the attractor-repulsor, the repulsive force would suffer an illicit compression, because the body would approach both foci simultaneously, and thus the balance would break in favor of the attractive force.

Consequently, the planet, once arrived in the perihelion, begins to move respecting a criterion only formally different, but substantially identical, to the previous one, namely the criterion of "minor possible distancing from the attractorrepeller".

The planet, after perihelion, manages to move away from the Sun as little as possible, because, as we said in the introduction, in the aforesaid point the two forces are exactly opposed and therefore the planet moves for a single moment of straight motion, since, only by moving itself in rectilinear way for that one instant, the planet 
can cease to approach the Sun and, at the same time, begin to move away from it as little as possible.

In the phase of distancing from the attractor-repulsor the planet is pushed, among all the points having a minor energetic value existing in its surroundings, towards the less weak one.

In geometrical terms, the planet, in the phase of distancing from the Sun, is pushed, among all the points farther from the Sun existing in its surroundings, towards the less distant one.

By following this principle, the planet moves away from the central body as little as possible.

As we can always see in the figure done in the paragraph 1.3, the planet, from perihelion, moves to $\mathrm{E}$ (only for clarity reasons the point $\mathrm{E}$ has been drawn slightly moved by perihelion, but it must be imagined as contiguous to it): this point is, at the same time, farther from the Sun with respect to the perihelion itself, but, among all the farther points from the Sun existing in the perihelion's surroundings, it is the less distant.

By following the same criterion, the planet will move from point $\mathrm{E}$ to point $\mathrm{F}$, and so on, until reaching the aphelion, in the most gradual manner possible, so as not to violate the principle of energy conservation, which would be injured if the body moved away from the attractor-repulsor in an insufficiently gradual manner, thus suppressing the attractive force.

\section{But how can a body A move away from a B body as little as possible?}

The only way is to move A on an elliptical trajectory in which B is in one of the foci, and the second focus is as close as possible to B.

In this way A will follow a trajectory characterized by the fact that, while moving away from B, it approaches at the same time as little as possible a point very close to B (the other focus), because, as mentioned above, "the ellipse is the geometric place of the points of plan where every point, compared to the adjacent one, moves away as little as possible from a focus and, at the same time, approaches as little as possible the other focus": in this way A will move away from B as little as possible, since, while it does so, approaches at the same time as little as possible a point (the 
other focus) very close to B.

However, the principle of the "minor possible distancing" from the attractorrepulsor is not applicable throughout the planet's orbit.

It's reasonable to suppose, in fact, that in an attractive-repulsive force-field generated by a central body, the existence of the two opposing forces causes the minor possible distancing to take place only up to a certain limit, beyond which, the further distancing from the attractor-repulsor would violate the principle of energy conservation, by suppressing the attractive force.

In my opinion, the point where this particular extreme situation would occur, is, in the planetary orbits, the aphelion, beyond which, if the body continued to move away from the attractor-repulsor, the attractive force would be suppressed, since the body would move away from both the foci, and so the balance would break in favor of the repulsive force.

Therefore the planet, once arrived in aphelion, begins to move again according to the principle, previously stated, of the minor possible approach to the attractorrepulsor, and the cycle repeats itself to infinity.

It's opportune to specify that in aphelion, unlike the perihelion, the two forces do not cancel each other out, because otherwise the planet would continue to move away from the Sun, in such a way to suppress the attractive force, so that the repulsive force continues to have a non-radial orientation, but this time it points more towards inside of the orbit, allowing the planet's approach to the Sun.

Basically, the planet would be able to revolve in perpetuity around the central attractor-repulsor because, approaching as little as possible to it and then distancing as little as possible from it, it remains in a state of perfect dynamic balance between the two opposed gravitational forces, thus preserving the principle of energy conservation.

The existence of this principle is confirmed by the fact that most of the planets (7 on 9) have orbits with very slight eccentricity, i.e. with the foci very close to each other. Only Mercury and Pluto have a non-negligible eccentricity, most likely because they come from outside the solar system (like the asteroids), as their little dimensions would suggest. 


\section{- 1.15 - The gardener's ellipse}

To confirm the above, just consider that the ellipse can be defined as "the geometric place of the points of the plan existing around two fixed points called foci, where each point, with respect to the adjacent one, approaches as little as possible a focus and, at the same time, moves away as little as possible from the other focus".

This definition is not substitutive, but alternative to the traditional notion of the ellipse understood as "the geometric place of the points of the plane for which the sum of the distances from two fixed points called foci is constant", but thanks to it it's possible to observe the ellipse from a whole new perspective, dynamic if we want, fundamental to understand the reason why the orbits of the planets of our solar system are elliptic.

\section{- Demonstration}

The demonstration of the validity of the alternative definition of ellipse seen above may appear rudimentary, but, in my opinion, is efficacious.

In the drawing below we see a gardener intent on tracing an ellipse following a method often ignored, but valid.

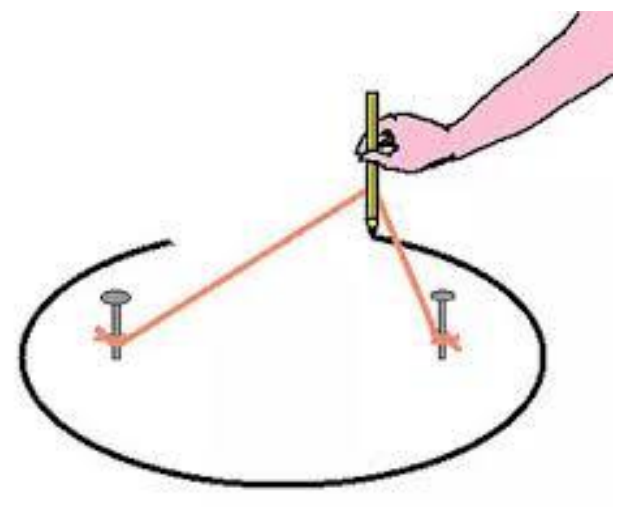

He has fixed two nails in the ground and tied to them a thread longer than the distance between the nails.

With a pencil he has exerted a pressure outwards to stretch the thread and, at the same time, has moved the pencil sideways to trace the ellipse.

The important thing to note in this drawing is that the forces involved are two:

1) the outward force exerted by the gardener with the pencil, which generates the tension of the thread. 
2) the reaction force generated by the wire itself.

Because of the thread, the gardener is bound in tracing the drawing: while he does it, he moves away as little as possible from a focus and, at the same time, approaches the other focus as little as possible.

As he moves, in fact, the string forces him to approach a nail and, at the same time, to move away from the other nail, because the wire is tied to the two nails, therefore, when the distance from a nail increases, the distance from the other one must necessarily be reduced, otherwise the thread would break, which allows us to conclude that it is not possible for the gardener to move away from both foci at the same time.

On the other hand, if the gardener's pencil approaches the two foci at the same time, the thread would loosen too much, and the tension of the thread, necessary to make the ellipse, would disappear.

We can therefore affirm that the ellipse is the graphical representation of the gardener's pencil movement, whom motion gives rise to two simultaneous movements with respect to the two foci, moving away as little as possible from a focus and, at the same time, approaching as little as possible the other focus.

Excluding the forces, irrelevant in the geometrical field, we can use the mentioned property of the ellipse to prove that every point of the ellipse always meets two conditions, i.e. that, compared to the adjacent point, it approaches as little as possible a focus and, at the same time, moves away as little as possible from the other focus, so we can affirm that "the ellipse is the geometric place of the points of the plan existing around two fixed points called foci, where each point, compared to the adjacent one, approaches as little as possible a focus and, at the same time, moves away as little as possible from the other focus".

The gardener's ellipse, therefore, shows that the trajectory described by a body inserted in an attractive-repulsive forces-field cannot be anything other than elliptical, since the two forces operating in the ellipse of the gardener can just be compared to the two opposite forces that characterize an attractive-repulsive forces-field, and this is of fundamental importance for the demonstration of the existence of such a kind of forces field around the Sun and all the massive bodies. 


\section{- 1.16 - Energy of the attractive-repulsive gravitational field}

When I talk about the energy of the attractive-repulsive gravitational field, I refer, therefore, to a particular type of energy inherent only in the attractive-repulsive fields (such as the one that most probably also exists around the nucleus of the atom, generated by the nucleons), which expresses the aptitude of a determined point of the field to move any body inserted in it, and having a negligible mass with respect to the central body, towards the point which, among those endowed with greater energy (or pressure) than the one existing in the occupied point, is the point with less-strong energy, or to make it move towards the point that, among those with less energy (or pressure) than the one existing at the point occupied, is the point with less-weak energy, depending on whether they are respectively in the approaching phase or in the distancing one with respect to the attractor-repulsor.

This energy (or pressure) would be decreasing in relation to the increase of distance from the center and we'll see in the chapter V how much important this aspect is for the solution of many astrophysics and nuclear physics problems.

\section{- 1.17 - Difference between field of inexistent forces and field of self- canceling out forces}

The difference between the two types of field consists in the fact that, in the first case (field of inexistent forces), the space is absolutely devoid of forces, whereas, in the second case, the space is pervaded by opposing forces (convergent or divergent) that cancel each other out.

The difference is not merely formal, because, in the second case, a body immersed in the field of forces can undergo physical changes due to the effect of the two opposing forces acting on it (crushing, deformation).

This clarification is important to eliminate any doubts that may arise regarding the existence of the attractive-repulsive force-field around the Sun which, it is repeated, does not equate to a field of null forces.

- $\quad 1.18$ - Aphelion as the point from which the motion of the planets has begun

If it is agreed that the cause of the movement of the planets is the existence of 
an attractive-repulsive gravitational field that generates a particular type of pendulum that moves not by oscillating, but by making turns around the fulcrum, then it will also be easy enough to admit that the point from which the motion of the planets has begun, probably already since the moment they were in a state of protoplanetary dust, is the aphelion, that is the point of the orbit in which the gravity force is less, similarly as the pendulum, in which the motion starts precisely from the point where gravity is less.

- 1.19 - The necessary ellipticity of the orbit of the celestial bodies around a central body

Such a vision of the phenomenon of the perpetual motion of celestial bodies, in which motion obeys the principle stated above (of the least possible approach and of the least possible distancing from the attractor-repulsor) also requires us to believe that in all gravitational systems (even dissimilar to ours, that is, when the system's bodies have not a negligible mass with respect to each other, for example a system of two binary stars), only elliptical orbits are possible.

\section{Chapter II}

Mathematical demonstrations

$* * *$

- $\quad 2.1$ - Demonstration of Kepler's third law by hypothesizing that the solar system is a harmonic oscillator 
As I said before, the period of planetary orbit around the Sun is expressed by the following formula, discovered by Galileo with regard to the harmonic pendulumoscillator, but applicable, as will be seen, also to the solar system,

$$
T=2 \pi \sqrt{\frac{L}{g}}
$$

where I assumed that $L$ is the average distance of the planet from the Sun and $g$ is the repulsive gravitational acceleration exerted on the planet by the Sun (i.e, when it is at the average distance from the star), so the (3) becomes:

$$
T=2 \pi \sqrt{\frac{a}{g_{r(\text { average })}}}
$$

where $a$ is the semimajor axis of the elliptical orbit of the planet which, as is known,

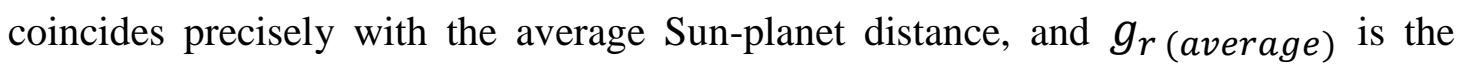
average repulsive gravitational acceleration exerted on the planet by the Sun, i.e. when the planet is at average distance.

But, since we have shown that in the cosmic harmonic oscillator, as in the Galilean one, centripetal acceleration does not accumulate to the gravity acceleration, we have that $g_{r}=-g_{a}$, and therefore (4) becomes:

$$
T=2 \pi \sqrt{\frac{a}{g_{\text {a(average })}}}
$$

where $g_{a \text { (average) }}$ is the average attractive gravitational acceleration, i.e, the one exerted by the Sun when the planet is at the average distance.

In this regard it should be noted that it was considered necessary to include in the (5) the average planet-Sun distance, as well as the average gravity acceleration, because, being a pendulum with an elliptical orbit, the distance from the fulcrum changes continuously (unlike the Galilean one, which has a circular orbit) and, consequently, also the gravitational acceleration deriving from the Sun varies continuously, so the insertion of the mentioned average values seemed to me the most logical thing to do.

But we also know that:

$$
g_{a(\text { average })}=\frac{G M}{a^{2}}
$$

so (5) becomes: 


$$
\begin{gathered}
\mathrm{T}=2 \pi \sqrt{\frac{a}{\frac{G M}{a^{2}}}} \\
\rightarrow \mathrm{T}=2 \pi \sqrt{\frac{a^{3}}{G M}}
\end{gathered}
$$

Squaring the both sides:

$$
T^{2}=\frac{4 \pi^{2} a^{3}}{G M}
$$

Dividing both sides by $a^{3}$ we have:

$$
\frac{T^{2}}{a^{3}}=\frac{4 \pi^{2}}{G M}
$$

which is precisely the third Kepler's law.

We have therefore shown that the Kepler's third law depends on gravity force, assuming that the solar system is a harmonic oscillator, and that, therefore, there is a high probability that repulsive gravity force exists in addition to the attractive one, such as the equality among the planetary laws (of orbital period, of angular velocity and of acceleration) with respect to the same laws of the harmonic oscillator would suggest.

\section{- $\quad 2.2$ - Demonstration that the planetary system is a harmonic oscillator}

We know that the pulsation of a harmonic oscillator (its angular velocity) is:

$$
\omega=\sqrt{\frac{g}{L}}
$$

Now, if the solar system is a harmonic oscillator, (11) it should become:

$$
\omega=\sqrt{\frac{g}{a}}
$$

where $a$ is the semi-major axis.

We must therefore prove that the above equation is true.

We know that the angular velocity, in the circular motion, is:

$$
\omega=\frac{2 \pi}{T}
$$

But, in planetary motion, the period $\mathrm{T}$ is:

$$
T=2 \pi \sqrt{\frac{a^{3}}{G M}}
$$


so:

$$
\omega=\frac{2 \pi}{2 \pi \sqrt{\frac{a^{3}}{G M}}}
$$

From which:

$$
\omega=\frac{1}{\sqrt{\frac{a^{3}}{G M}}} \rightarrow \omega=\sqrt{\frac{G M}{a^{3}}}
$$

But we also know that:

$$
g=\frac{G M}{a^{2}} \rightarrow G M=g a^{2}
$$

Therefore, replacing the (17) in (16), we have:

$$
\begin{aligned}
\omega & =\sqrt{\frac{g a^{2}}{a^{3}}} \\
\rightarrow \omega & =\sqrt{\frac{g}{a}} \quad \text { C. V. D. }
\end{aligned}
$$

Let us now demonstrate that acceleration in planetary motion follows the acceleration law of the harmonic oscillator of Galileo, namely:

$$
g=\omega^{2} x
$$

Where $x$ is the position of the body, namely its projection on the abscissas axis.

By assuming that the solar system is a harmonic oscillator pendulum with radius equal to the semi-major axis $a$, and comparing the orbital ellipse to circumference, as Newton did, then in planetary motion it should occur, if such assumption is correct, that:

$$
g=\omega^{2} a
$$

Now let's move on to the demonstration.

In (19) we have seen that the angular velocity of the planets is equal to the pulsation of the harmonic oscillator:

$$
\omega=\sqrt{\frac{g}{a}}
$$

Squaring we have:

$$
\omega^{2}=\frac{g}{a}
$$




$$
\rightarrow g=\omega^{2} a \quad \text { Q. E. D. }
$$

To understand whether (23) applies only to a point in the orbit or to all points of it - in which case it would rise to the acceleration law of the motion of the planets - it must be verified experimentally.

Now, by means of the aforementioned formula, we will first calculate the gravity acceleration in perihelion and then the one in aphelion, and it will be shown that it allows to calculate precisely both, which will attest the acceleration law nature of (23), i.e. of law that regulates the overall movement of the planets throughout the orbit, not just in a single point.

To this end we will choose the orbit of Mercury which is characterized, due to its high eccentricity, by a perihelion and an aphelion very different from each other, both in terms of distance from the Sun (and therefore of gravity acceleration), and in terms of speed difference compared to the average orbital speed.

\section{- Mercury's perihelion}

We now calculate the gravity acceleration in perihelion using (23) and see if it is equal to the gravity acceleration calculated with its specific formula:

$$
g=\frac{G M}{(\text { perihelion })^{2}}
$$

To this end we must calculate the angular velocity existing in perihelion, which is not the average velocity, because the planet's orbital velocity varies continuously, and in the perihelion the velocity is maximum.

Consequently I thought that the best thing to do was to calculate the radius of the osculating circle in perihelion (which, as is known, is the circle that approximates in the best way the curvature existing in a given point of a non-circular curve), to be used later to calculate the value of $\mathrm{T}$ in the angular velocity formula:

$$
\omega=\frac{2 \pi}{T}
$$

From the basic formula of speed we know that:

$$
T=\frac{S}{V}
$$

But we also know that, in the case of circular motion:

$$
S=2 \pi r
$$


Where $r$ must be, as we have said, the radius of the osculating circle which, in perihelion, is:

$$
r=a\left(1-e^{2}\right)
$$

Where $a$ is, as we know, the semi-major axis, and $e$ it is eccentricity.

By knowing that the semi-major axis of the orbit of Mercury is:

$$
a=57,909,176,000 \text { meters }
$$

and that the eccentricity of Mercury is:

$$
e=0.20563069
$$

the radius of the osculating circle in the perihelion of Mercury will be:

$$
r=57,909,175,999 \text { meters }
$$

We now replace in (26) the value of $r$ just found:

$$
S=363,854,083,793 \text { meters }
$$

We also know that in perihelion the orbital velocity of Mercury is:

$$
V=58.980 \mathrm{~m} / \mathrm{sec}
$$

We now replace in (25) the values of $S$ and $V$ found above:

$$
T=\frac{363,854,083,793}{58,980}=6,169,109
$$

We now replace in (24) the value of $\mathrm{T}$ just found:

$$
\omega=\frac{2 \pi}{6,169,109}=0.000001018491
$$

Now let's replace in (23) the value of $\omega$ just obtained, let's square it and multiply it by the semimajor axis $a$ and we'll get:

$$
g=0.06007 \mathrm{~m} / \mathrm{sec}^{2}
$$

We now calculate the gravity acceleration due to the attraction that the Sun exerts on Mercury in perihelion using the known formula:

$$
\mathrm{g}=\frac{G M}{{\text { (perielio })^{2}}^{2}}
$$

Where $G$ is the universal gravitational constant $6.67433 * 10^{-11}$

$M$ is the mass of the Sun: $1.9891 * 10^{30} \mathrm{~kg}$

The perihelion of Mercury: 46,001,272,000 meters

We replace in (29) the values of $G, M$ and perihelion reported above:

$$
\mathrm{g}=0.06273
$$


Let us now compare the two values of $g$ obtained in the eq. (30) and in the eq. (28):

$$
\frac{0.06273}{0.06007}=1.04
$$

As we note the values of $g$ found with the formula of the harmonic oscillator (23) and with the specific formula of gravity acceleration (29) are very similar.

It is very likely that the slight discrepancy is justified just because the calculation of the radius of the osculating circle is an approximation criterion which, as such, may be less precise when the numbers involved are very large, such as astronomical ones.

\section{- Mercury's Aphelion}

At this point, always by using the formula of the harmonic oscillator obtained in (23), let's calculate the gravity acceleration in aphelion and let's see if it is equal to the gravity acceleration calculated with its specific formula $g=\frac{G M}{(\text { afelio })^{2}}$.

To this end, we proceed in the same way seen as for perihelion.

We have to calculate, therefore, the angular velocity existing in aphelion, which is not the average one, because the planet's orbital velocity varies continually, and in aphelion the velocity is minimal.

Consequently we calculate the radius of the osculating circle in aphelion (which, it is repeated, is the circle that approximates in the best way the curvature existing in a determined point of a non-circular curve), to be used then to calculate the value of $\mathrm{T}$ in the angular velocity formula:

$$
\omega=\frac{2 \pi}{T}
$$

From the basic formula of speed we know that:

$$
T=\frac{s}{V}
$$

But we also know that, in the case of circular motion:

$$
S=2 \pi r
$$

Where $r$ must be, as we have said, the radius of the osculating circle which, in aphelion, is equal to the perihelion, because in the two points the curvature is identical, so the value of the aforementioned radius is the one calculated in perihelion: 


$$
r=57,909,175,999 \text { meters }
$$

We now replace in (33) the value of $r$ just found:

$$
S=363,854,083,793 \text { meters }
$$

We also know that in aphelion the orbital velocity of Mercury is:

$$
V=38,860 \mathrm{~m} / \mathrm{sec}
$$

We now replace in (32) the values of $S$ and $V$ found above:

$$
T=\frac{363,854,083,793}{38,860}=9,363,203
$$

We now replace in (31) the value of $\mathrm{T}$ just found:

$$
\omega=\frac{2 \pi}{9,363,203}=0.000000671
$$

Now let's replace in (23) the value of $\omega$ just obtained, let's square it and multiply it by the semi-major axis $a$ and we'll get:

$$
g=0.02607 \mathrm{~m} / \mathrm{sec}^{2}
$$

We now calculate the gravity acceleration due to the attraction that the Sun exerts on Mercury in aphelion by using the known formula:

$$
\mathrm{g}=\frac{G M}{(\text { afelio })^{2}}
$$

Where $G$ is the universal gravitational constant $6.67433 * 10^{-11}$

$M$ is the mass of the Sun: $1.9891 * 10^{30} \mathrm{~kg}$

the aphelion of Mercury is 69,817,079,000 meters

We now replace in (36) the values of $G, M$ and aphelion reported above:

$$
\mathrm{g}=0.02723 \mathrm{~m} / \mathrm{sec}^{2}
$$

Let's now compare the two values of $g$ obtained in (37) and in (35):

$$
\frac{0.02723}{0.02607}=1.04
$$

As we note the values of $g$ found with the formula of the harmonic oscillator (23) and with the specific formula of gravity acceleration (36) are very similar.

It's very likely, again, that the slight discrepancy is justified in light of the fact that the calculation of the radius of the osculating circle is an approximation criterion which, as such, may be less precise when the numbers involved are very large, such as astronomical ones. 
It should be noted that by the application of (23) it's possible to obtain identical results also for the other planets, which, however, is not the case here to report in order to synthesize.

\section{- Summary consideration}

By having calculated with precision by mean of (23) the gravity acceleration at two points of the orbit of Mercury where such acceleration assumes very different values, due to the considerable diversity of the two distances from the Sun, we can deduce that (23) is the formula that expresses the acceleration not of a single point of the orbit of the planets, but of all the points of this orbit, albeit with the specification that we have to calculate the angular velocity existing at the specific point through the system of osculating circle, because in the planetary harmonic oscillator the angular velocity is not constant, by varying point by point both $g$ and the curvature of the orbit.

It should be noted that it was possible to use in (23) the semi-major axis $a$ in order to calculate $g$ both in perihelion and in aphelion, because, as it is known, in these two points the amplitude of the harmonic oscillation is maximum, so that such amplitude coincides with the radius which, in our case, in turn coincides with the semi-major axis $a$, by we having approximated the orbits of the planets to the circumferences.

It follows that, when we want to calculate by (23) the gravity acceleration at a certain point of the orbit other than perihelion and aphelion, it will be necessary not only to calculate the radius of the osculating circle at that point, but also the $x$ position of the body, i.e. its projection on the abscissas axis.

It follows that we can replace in (23) $a$ with position $x$ and write:

$$
g=\omega^{2} x
$$

that is the acceleration law of the pendulum harmonic oscillator seen in (20).

As we wanted to demonstrate, the solar system is a case of harmonic oscillator, obviously much more similar, if not exactly the same, to the pendulum, rather than the spring. 
The most important consequence of this is the demonstration of existence, starting from a certain distance from the Sun (and from all the massive bodies), of a repulsive gravitational force, generated always by the Sun itself in addition to the attractive one, which takes the place of the Earth's gravity force existing in the Galilean pendulum, thus pushing the planets away from the Sun, albeit with an orientation not opposite to the attractive one, guaranteeing the perpetual motion of the planets around the Sun, according to the same principle as the pendulum.

This is even more true if we consider that the harmonic oscillators are all characterized by the fact that the body moving by non-uniformly accelerated and decelerated motion is continuously subjected to two contrasting forces which, although in different ways, guarantee the potentially perpetual movement of the sphere hanging from the pendulum or the spring.

Unlike the harmonic motion, the uniform circular motion, which never occurs in celestial mechanics, is characterized by the presence of a single central force.

\section{- 2.3 - The accelerated expansion of the universe explained by the exclusively repulsive effect of gravity}

But at this point we ask: what happens if we go beyond the space band where there exists the contrast between attractive and repulsive force?

It is really far-fetched, in fact, to believe that the dynamic balance between the bodies, due to the clash between the two opposed forces (one attractive and the other repulsive), which allows the perennial movement of one around the other according to the law of pendulum, may always apply, regardless of the distance between the bodies themselves.

Therefore I hypothesized in this regard that, as below a certain distance from the body attractor the repulsive force ceases to exist, giving space to the only attractive force, similarly it can be considered that, when a body exceeds a certain distance from the attractor, the attractive force ceases to exist, and it will give space to the only repulsive force, and that, in this case, it is also consistent to assume that, as in the attractive zone the body moves with uniform accelerated motion, similarly the body moves with uniform accelerated motion even when it is in the exclusive 
repulsion zone, pushed only by repulsive force.

This deduction seems to me to be confirmed by the accelerated expansion of the universe, which is receiving more and more experimental confirmations and consequent adhesions from the scientific community, although there is not agreement about the relative causes.

In particular it is believed, on the basis of very accurate astronomical observations, that galaxies repel each other, moving away from each other with increasing speed.

From this point of view, it is evident that the accelerated repulsive motion, which I hypothesized to be observable in the space band furthest from the body, is perfectly mirrored to the accelerated contractile motion in the proximity zone.

\section{Chapter III}

The ligamentous nature of gravity 


\section{- 3.1 - Gravitational ligaments}

We have seen that the accelerated expansive effect of the universe can be explained by the exclusively repulsive effect of the gravitational force, which manifests itself beyond the attractive-repulsive zone.

But how is it possible to believe that repulsion works indefinitely?

If we accept the idea of a repulsive force, we come to the inevitable conclusion that the body should progressively increase its speed until it reaches an infinite speed.

Now, leaving out for now whether it is possible for a body to accelerate indefinitely, the main question is how it is possible that the repulsive effect works indefinitely.

Logic would like, at a first and superficial analysis, that sooner or later, the distance becomes so high that the repulsive effect is exhausted, and therefore the body continues to move with uniform rectilinear motion.

Then, how is it possible for the repulsive effect to work on the infinite?

The only possible explanation is to believe that gravity force is not transmitted as an information, as a wave or other similar phenomena, but operates through an invisible ligament that connects the bodies between them, such as to make them become one.

The ligamentous nature of gravity is the only one that can explain, in my opinion, the phenomenon of the accelerated and potentially infinite expansion of the universe.

The gravitational ligament is also the only possible explanation of the fact that gravity operates in an immediate way, i.e. faster than light.

So if, for example, the mass of the Sun increases, the attraction (or rather the attraction-repulsion) that it exerts on the planets would have an immediate intensification, therefore faster than the speed of light, in disagreement with the principle of locality postulated by the theory of relativity.

This can have only one explanation: the ligamentous nature of gravity, which eliminates the transmission time of information.

In other words, hypothesizing that there is an invisible ideal ligament that unites two bodies, like an extendable and contractible bar joining two metal spheres, 
and that the elongation or contraction are immediate (precisely because it is an ideal ligament), is logical consequence that if the bar stretches, the distancing of the rejected body is immediate, and the approach of the attracted body when the ligament contracts himself is equally immediate.

For this reason it is inaccurate to say that the gravity force is transmitted between the bodies at a certain speed, because we can speak of information transmission speed only when the bodies between which the information travels are two, but the gravitational ligament makes sure to unite the two bodies in one, so any approach to the concept of speed is improper.

In this way we can also overcome the criticism of those who, including Einstein, believe that to think of gravity as an immediate force cannot be accepted, because otherwise it would be faster than light, which would be impossible.

By accepting gravity has ligamentous nature makes each approach to the concept of speed out of place, because of the abstract uniqueness of the bodies involved in the gravitational phenomenon, united through the gravitational ligament.

\section{- $\quad 3.2$ - Space-time network or gravitational ligaments network?}

Gravitational ligaments are potentially infinite, both in number and in extension.

Every body emanates as many ligaments as there are bodies in the entire universe.

Through these ligaments, each body of the universe is connected to all the others and the others connected to each other through a very intricate netting of gravitational ligaments, regardless of the distance that separates them.

None body is disconnected from anyone else.

The space is therefore pervaded by a kind of invisible and imponderable netting, but this does not take the form of the so-called fourth dimension hypothesized by Einstein (flexible space-time), instead by concretizing an intertwining of ideal bonds that do not bend close to the bodies, but, unlike the Einstein way of thinking, they remain absolutely straight, uniting the bodies together.

\section{- $\quad 3.3$ - Gravitational waves and their compatibility with ligamentous}




\section{nature of gravity force}

Recently it was discovered that the merging of two black holes occurred more than a billion years ago has generated gravitational waves that have come down to us after one billion years.

This would confirm, according to relativists, the existence of the fourth dimension hypothesized by Einstein, that is, the space-temporal network thanks to which the information of the disappearance of black holes merged into one would be transmitted.

More precisely it is stated that the detection of gravitational waves by a very sophisticated cryogenic instrument would constitute the indisputable proof of the existence of the space-time dimension, since only if we admit the existence of a spacetemporal network, we can explain how it is possible that gravitational waves propagate in space in a not immediate way, which instead could not happen, it is claimed, if we conceived gravity as a mysterious "instantaneous" force acting at distance between the bodies (Newton's theory), which, if it existed, it should disappear immediately with the disappearance of the black holes involved in the fusion, and it could not propagate in space at a distance of time, unlike what has been detected by the cryogenic instrument.

This statement would be fully acceptable if we believed, according to the Newtonian theory, that the only possible form of manifestation of the gravitational force is the one of an instantaneous attracting entity that operates at distance between the bodies, but falls if we adhere to the theory, here proposed, according to which the gravity force "binds" the bodies by uniting them into one, thus eliminating the distance between them.

In other words, by conceiving gravity force as an ideal ligament, we arrive at the logical consequence that when two black holes merge, the gravitational ligaments which they own, potentially infinite in quantity and length, undergo a change of ownership, transferring themselves from the two original holes to that deriving from the merger.

Since nothing is destroyed in nature and nothing is created, but everything is transformed or yielded, the gravitational ligaments carried by each individual black hole participating in the merger are, in my opinion, transferred to the black hole 
resulting from the merger, and most likely it is this transfer of "ownership" of the gravitational ligament node that generates gravitational waves, which this time operate not in an immediate way, but in a mediated way, namely as a means through which information about the change of ownership of the ligament node propagates.

With this in mind, it is more accurate to say that, with fusion, black holes do not disappear, but flow into the larger one, with the consequence, of no small importance, that the gravitational ligaments of which they were carriers do not disappear, but flow into the black hole resulting from the merger.

The phenomenon under scrutiny can be compared to that of a rope contended between two men who pull forcefully from the two opposite sides: if one of the two men suddenly gives a shock to the rope, the other will perceive the wave generated by the shaking not immediately, but after a few seconds, unlike the hypothesis in which one of the two men suddenly intensifies the force: in the latter case, by assuming that the rope is made up of perfectly inextensible material, the increase in strength would be perceived by the other man immediately, because the rope, being held in a state of tension by the opposing forces exercised continuously by the two men, acts as a unifying element of the two human bodies: it is as if the rope merges the two bodies into one.

Here is, then, the reason why the gravitational waves generated by the fusion of the two black holes occurred more than a billion years ago have been perceived by the cryogenic instrument after so much time: not because there is the space-time network through which they propagate, but because there is a network of gravitational ligaments that acts as an instrument for propagating these waves.

In other words, on the occasion of the merger of two black holes there is a change in ownership of the ligament node belonging to the individual black holes involved in the merger, and this change generates a sort of shaking of the ligaments which, as such, is not transmitted if not in a deferred way to the bodies (potentially infinite) that are on the opposite side of the same ligaments.

If we accept, therefore, the idea of gravitational ligament, it is possible to explain perfectly not only how it is possible that gravity operates in an instantaneous way even at infinite distances, as a force that "binds" the bodies together, but also how it is possible that it operates in a mediated way, as a transmitter of information 
(change of ownership of the ligament).

It is obvious that the gravitational ligament is not something that can be proven, it is not made up of particles of matter, and any attempt to prove its existence would give a negative result.

It is likely to be made up of "gravitons", as quantum mechanics defines them, but I distrust the existence of invisible particles.

In truth, quantum physicists believe that these particles are invisible because they are covered by Heisenberg's uncertainty principle, according to which it is not possible to observe infinitely small particles (like electrons) because the light beam that would be needed to observe them would continuously change them position.

However, I believe that the gravity force is not the consequence of an exchange of invisible particles from one body to another, but is the consequence of contraction or expansion of an invisible ideal ligament, so that, at most, if these invisible particles exist, they would dilate and they would contract, but certainly would not move, which makes Heisenberg's indeterminacy principle inapplicable, which is based precisely on the unpredictable movement of the infinitesimal particles.

It is not possible to give a logical, scientific, rational explanation to the mechanism that underlies the phenomenon of gravitational attraction-repulsion, and who has tried to do it, rejecting the idea of the mysterious force, must necessarily come up with hypotheses such as the curvature of the empty space, the contractility and dilatability of time which, to tell the truth, are no less alchemical and metaphysical of the mysterious force.

It is not possible to understand where this mysterious and invisible ligament derive from: it suffices to know that it derives from the body, which is its sole maker, which is already a great achievement. 


\section{force: differences}

$* * *$

\section{- $\quad 4.1$ - Definition of centripetal force and of centrifugal force}

The centripetal force, it's worth remembering, exists whenever a body performs a curvilinear trajectory due to a central force.

A car revolving around a roundabout, the clothes running in the washing machine, a ball tied to a rope made move around by a child are all hypotheses in which the rotating body is subjected to centripetal force.

In fact, when we use the washing machine, in order to be sure that at the end of the program the cloths receive a first drying due to the high final rotation speed of the washing machine drum, we activate the centrifugation function, but in this case it is operating not properly the centrifugal force, but the centripetal one, because, the real force acting on the clothes is centripetal, not centrifugal, but our mind perceives more immediately the existence of a centrifugal force on the clothes, rather than centripetal one, because we imagine that the expulsion of water from cloths results precisely from centrifugal force, not centripetal one. But it's not like that.

The force to which the cloths are subjected is centripetal, not centrifugal, because, if there was a big enough hole in the basket, the clothes would come out with along a tangential trajectory to the basket, not radial.

This happens because the basket opposes the straight movement of the clothes, preventing they from continuing straight in a tangential orientation to the circular trajectory.

The curve in fact is nothing but a set of infinitely small segments that change orientation continuously, and the reason why the cloths run attached to the washing machine spin basket without falling, is that the basket makes them do not go straight on. But the water succeeds in infiltrating the specially made holes in the basket, and continues straight ahead, in a tangential orientation to the basket.

Centrifugal force is only a "fictitious force" that we perceive in the car when we make a curve, which makes it seem like there is something that pushes us to the right if we curve to the left, or to the left if we curve to the right.

However, there is nothing that pushes us in the opposite orientation to 
curve's one, but it is only our tendency to preserve the straight motion that makes us perceive the presence of this fictitious centrifugal force; the only real force to which we are subjected is centripetal, that is, directed towards the center of the circle that we are describing while we are executing the curve, and which prevents us from going straight.

Well, as stated above, the magnitude of the centripetal force is measured by the following formula:

$$
F_{c}=m \frac{V_{t}^{2}}{R}
$$

Where $V_{t}$ is the tangential velocity of the body, ie the speed at which the car moves with respect to the road, with a tangent orientation to the trajectory; $R$ is the radius of the circle described by the car while it's turning; and $m$ is the mass of the car.

And centripetal acceleration?

From the second principle of dynamics we know that:

$$
F_{c}=m a
$$

So:

$$
a_{c}=\frac{V_{t}^{2}}{R}
$$

But what is centripetal acceleration, or rather what does it describe?

In general we know that acceleration is a variation in speed over a certain time interval.

The centripetal acceleration, however, does not express the variation of velocity of the body, that is the magnitude of the velocity vector, but expresses the speed with which the orientation of this vector changes.

We know that velocity is a vector quantity, and that the components of a vector are magnitude, orientation and sense.

Well, centripetal acceleration implies the change only in the orientation of the velocity vector, without altering the magnitude of the velocity, nor the sense.

It's quite intuitive to understand, regardless of the formula of centripetal acceleration seen above, that, by reducing the radius of the circumference described by a body in circular motion, and keeping the magnitude of the speed constant, the centripetal acceleration increases, because increases the speed with which the 
orientation of the tangential velocity of the body changes.

Otherwise, by increasing the radius, the centripetal force decreases, until almost completely zero if the speed is very small, like in the case of the harmonic oscillator with very long wire, where the very little amplitude of the oscillation, together with the very long length of the thread, determines the extreme slowness of the pendulum movement.

In the harmonic oscillator with very long wire, therefore, the centripetal force is absent or, if any, is so small as to become negligible.

\section{- 4.2 - Centripetal and centrifugal forces in the solar system}

But why all this rant about the centripetal force in the pendulum?

Because even in the cosmic pendulum, that is, in the solar system, the centripetal force does not affect the magnitude of the gravitational attractive force exerted by the Sun on the planets, for the reasons I will say shortly, so that the equality also remains valid here, like in Galilean harmonic oscillator, between central force (attractive gravity) and force that pushes the planet outward (repulsive gravity), which I suppose to exist in the solar system.

At this point it's necessary to remember that I have hypothesized above that the solar system is a harmonic oscillator pendulum, where the planet would be the sphere hanging from the wire; the gravity force that the Sun exerts on the planets would take on the same function as the centripetal force in the Galilean pendulum; and the repulsive force, which I imagine to be produced by the Sun starting from a certain distance from it in addition to the attractive one, would have the same function as the gravity force of the Galilean pendulum, which pushes the sphere in a opposite orientation with respect to the fixed point.

The only differences with the Galilean pendulum would be, firstly, that, in the cosmic pendulum, the central force is an action force, implying the elliptical orbit, unlike the classical pendulum, where the central force is a reaction force, implying the circularity of the trajectory; a second difference is that in the cosmic pendulum the external gravity force continuously changes its orientation always following the orientation of the planet's motion, and this change of orientation occurs with a speed equal to repulsive gravity acceleration (in turn equal to the 
attractive one), i.e. to the initial rapidity with which the planet would move away from the Sun if it were still and if the repulsive force was the only operating force, which does not happen in the Galilean pendulum, where the gravity force acting on the sphere always points towards the center of the Earth, with the consequence that the latter oscillates from one side to the other, whereas the cosmic pendulum makes a complete revolution around the center.

By having made this necessary premise on what on my opinion is the solar system, i.e. a harmonic oscillator pendulum with very long wire, with central attractive force, and with external gravitational force having changeable orientation and magnitude always equal to the first one, becomes easier to understand why in the cosmic pendulum the centripetal force does not influence, like in the Galilean harmonic oscillator with very long wire, on the magnitude of the attractive gravitational force.

In the cosmic pendulum the central force, we repeat, is attractive, and is measured by the following famous formula:

$$
F=G \frac{M * m}{R^{2}}
$$

where $G$ is a constant value (called gravitational constant), $M$ is the mass of the Sun, $m$ is the mass of the planet, and $R$ is the planet-Sun distance.

The central gravitational force, by being an attraction one, implies, as its indirect effect, also the impediment to the distancing.

In other words, the Sun attracts the planets towards it but, in while doing so, it also prevents from their distancing from it: in this sense the gravitational force is "also" a constraint, but it is not only that, since it contains an additional requirement compared to the simple reaction force, i.e. the ability to bring bodies closer together, by materializing in that attractive power lacking in the case of pivot on the table that we see on the next page. 


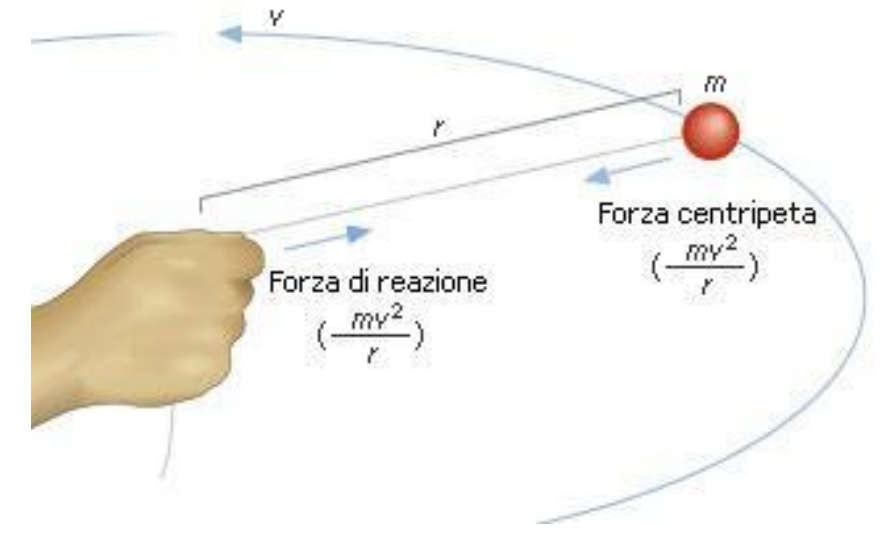

If we imagine that the hand is the pivot, we cannot say the ball is attracted by the pivot, so that, if we stop the ball, it remains where it is, it does not come close to the hand.

Now, leaving aside the force perceived by the hand, the important thing is that the hand, through the wire, exerts only a reaction force that prevents the ball from distancing in a straight line from the hand itself, which in turn generates centripetal acceleration, i.e. the change of ball speed orientation, but certainly not the approach of the ball to the hand, which will maintain a constant distance from it, describing a circular trajectory.

From this argumentation, we can understand why centripetal acceleration, that the gravitational attractive force produces indirectly on the movement of the planets, is generally not added to the gravitational acceleration itself, except in perihelion, as we shall see.

If we are agree that the centripetal force prevents the body from distancing in a straight line from the center, and that the centripetal acceleration, when the central force is attractive, is an indirect effect of the latter force (because whom attracts a body, indirectly also prevents it from moving away from the body), then it's obvious that centripetal acceleration cannot be summed to the direct effect of the attractive force, i.e. the attractive gravity acceleration, since they are two distinct effects of a single force: the difference between two effects is that the attractive gravity acceleration is directed towards the Sun, whereas the centripetal one is directed towards the center of curvature, i.e. the center of the osculating circle at that point, as seen in the drawing in following page (in which we have omitted to represent the repulsive force to make clear what happens inside the 
orbit), where $a_{n}$ is the centripetal acceleration, orthogonal to the orientation of the motion.

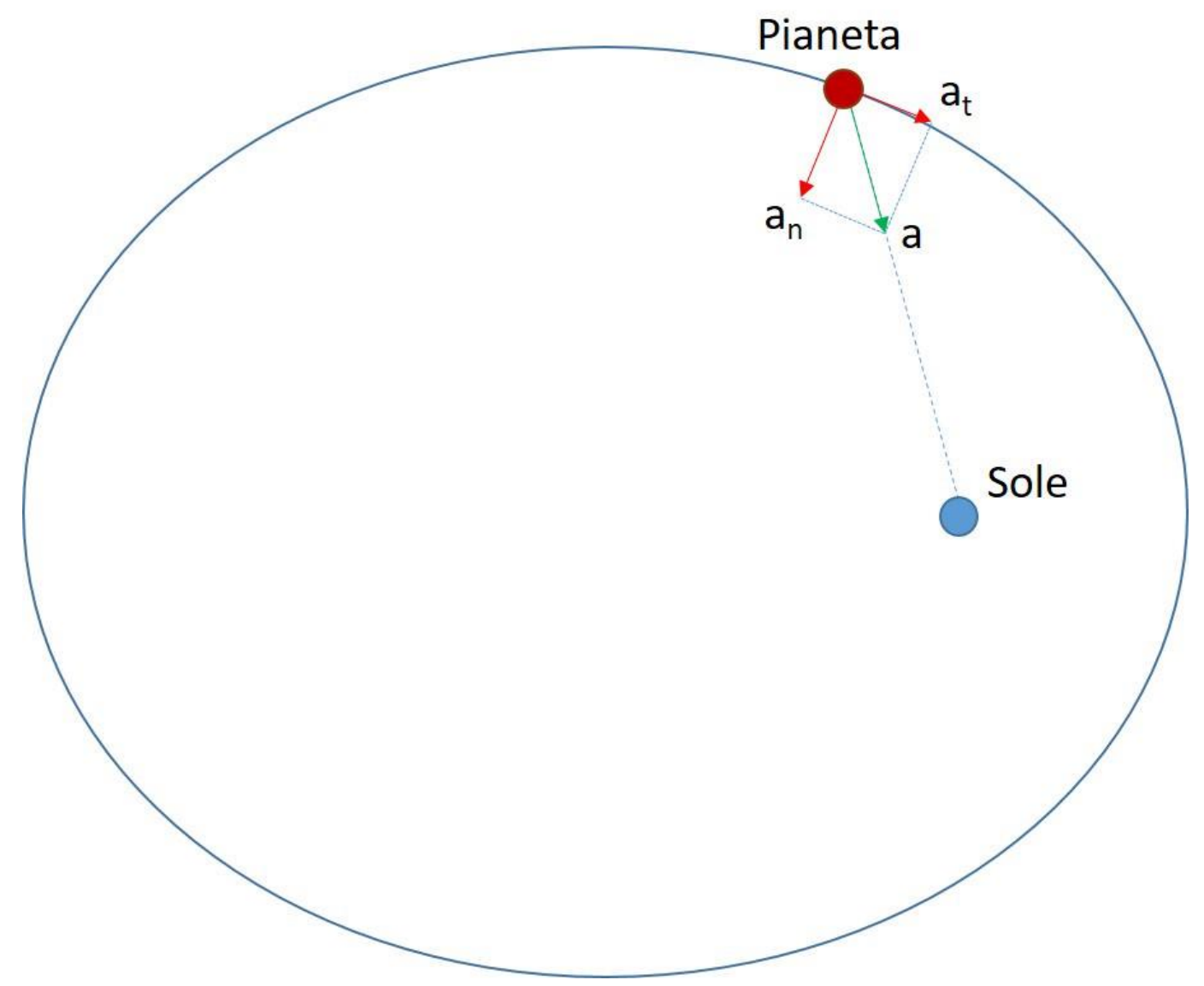

In confirmation of the fact that the planets are subjected to the combined attractive-centripetal effect, one can assert that they do not always keep the same distance from the Sun, as they would do if gravity behaved like a rope (which only generates centripetal acceleration), but, in while they revolve, they approach and move away from the star (depending on whether, respectively, they are heading towards perihelion or towards aphelion), and this dual contemporary movement (of revolution and approach/distancing) is caused, precisely, by the combined effect of attractive gravity acceleration and centripetal acceleration, on the one hand, and of repulsive gravity acceleration and centrifugal acceleration, on the other.

Unlike centripetal acceleration, centrifugal acceleration has a radial orientation - similar to the vector in the drawing on p. 2 representing the component $F_{r y}$ of the repulsive force - that is, stays on the line that joins the 
planet with the Sun (even if it is improper to speak of radius, since the orbit is an ellipse, not a circumference, but this term has only been used for practicality).

If, in fact, we admit that the repulsive gravity force exists, and that it changes every moment its own orientation following the trajectory of the planet, we have as consequence the birth of centrifugal acceleration, this time "real", not fictitious, because there is a real force (repulsive gravity), deriving from the Sun which, pushing the planet outwards, opposes the attractive force, and which changes continuously its orientation, with the same acceleration with which the planet is repelled by the Sun itself.

In the cosmic pendulum, therefore, the trajectory of the planet varies not only as the result of the centripetal acceleration (which prevents from the distancing in a straight line from the Sun), but also as the result of centrifugal acceleration (which prevents from the approach in a straight line to the Sun), which gives rise to the same effect as the centripetal one, i.e. the change in the orientation of the planet's trajectory over a given time interval.

The orientation of the repulsive force varies with the same acceleration with which the Sun repels the planet, because the tangential velocity vector and the repulsive force vector originate in the same point, i.e. the planet.

Basically, to create an image more like the pendulum that we know, it's as if the Earth did not remain still under the pendulum but, while it attracts this latter, changed position by moving along with the sphere, with the same orientation and with the same acceleration with which attracts it.

We have all the elements, therefore, to define the "real" centrifugal acceleration: it is the effect, indirectly caused by the central repulsive force, or by an external attractive force, which, by opposing the central attractive acceleration, prevents the body from approaching straight the center, making it move around it (whom rejects a body away from the center or attracts it from the opposite side to the center, indirectly prevents it from approaching the center itself).

Centrifugal acceleration, therefore, must be understood in an exactly specular sense with respect to the centripetal acceleration - which we have seen can be defined as the effect indirectly resulting from the attractive gravitational force, which prevents the planet from moving away from the Sun - although 
generating the same effect, that is the continuous change of the body's tangential velocity orientation, making it move around the center.

The first requirement for the birth of centrifugal acceleration is, first of all, that the body must be subjected to an attractive force towards the center, in the whose absence, obviously, it's impossible to have an impediment to approach the central body and, secondly, it must be subjected simultaneously to a central repulsive force or, alternatively, to an external attractive one which, by not having a radial orientation, opposes the attractive force without canceling out themselves.

The lack of the first requirement is the reason why in the Galilean pendulum, unlike the cosmic pendulum, the centrifugal acceleration is absent, since the sphere is pushed not towards the center, but far from it (due to the Earth's gravity): since the attraction towards the center lacks, we have the consequence that the impediment to approach the center, which is the typical effect of centrifugal force, can only be lacking, and only centripetal acceleration will exist, whose effect is to avoid the distancing from fixed point, making the sphere move around it.

What is important to clarify, is that we must not make the mistake of thinking that when the planet is approaching the Sun, the attractive force prevails, whereas, when it is moving away from it, the repulsive one prevails.

In fact, between the two forces there is always absolute equilibrium: what changes is only the orientation of the force $F_{r x}$, which is direct more towards the inside of the orbit when the planet is approaching the Sun, and more towards the outside when the planet is moving away from it.

This is important to understand that centripetal acceleration always exists, even when the planet is moving away from the Sun, and so also for the centrifugal one, which exists even when the planet is approaching the star.

It's also important to say that the centripetal acceleration and the attractive gravity acceleration are misaligned in all the points of the orbit, except in the perihelion: here the two accelerations are aligned, as well as, from the outer side of the orbit, the centrifugal acceleration and the repulsive gravity acceleration are aligned.

In addition to being aligned, the aforesaid 4 accelerations are also equal in magnitude, because the gravity accelerations, both attractive and repulsive, are 
orthogonal to the motion, whereby the attractive gravity acceleration becomes equal to the centripetal acceleration, and the repulsive gravity acceleration becomes equal to the centrifugal acceleration, so we can write the following equation, valid, we repeat, only for perihelion:

$$
a_{g a}=a_{c p}=a_{g r}=a_{c f}
$$

where:

$a_{g a}$ is attractive gravity acceleration;

$a_{c p}$ is centripetal acceleration;

$a_{g r}$ is repulsive gravity acceleration;

$a_{c f}$ is centrifugal acceleration.

The accelerations were represented without the above symbol of the vector, because, by being all aligned, it's possible to operate algebraically by adding or subtracting their magnitude. 


\section{Chapter V}

\section{Perihelion precession, black hole radius, atomic nuclear radius, radius of neutron star and stars orbital speed}

$* * * *$

\section{- 5.1 - Perihelion precession}

The assumption of the existence in the perihelion of four equal accelerations operating on the planets and summable algebraically (internal couple plus external couple), by each couple of forces having the same orientation and sense (the centripetal one added to the attractive gravity, and the centrifugal one to repulsive gravity), reverberates its effects, as we shall see, on the perihelion precession phenomenon, because of the particularity of this point.

As mentioned earlier, in the perihelion it's possible to sum the centripetal acceleration to the attractive gravity acceleration because the latter is orthogonal to the motion, so it does not split into tangential component and normal component to the motion, but both components have orientation turned towards the sun; similarly, we can sum centrifugal acceleration to the repulsive gravity acceleration, because the latter is not splitted into radial component and tangential component, but both components have the same direction: radial (i.e. both lying on the extension of the Sun-planet axis).

At this point, placing us in clear break with the Newtonian theory, where gravity is conceived of nothing more than a centripetal force, thus generating only centripetal acceleration, not also attractive gravity acceleration, it must be clarified, if it has not been done sufficiently until now, that here a clear conceptual split was made between the two accelerations (of attractive gravity and centripetal one), by having two different effects, as we have already said above, and the reasons for this split are identical to those on which the distinction between repulsive gravity acceleration and centrifugal acceleration is based: it was only thanks to this conceptual separation between the two accelerations that it was possible to sum them in perihelion.

The bizarre thing, however, is that, in the perihelion of the cosmic pendulum, it occurs not only that the centripetal acceleration is summable to attractive gravity 
acceleration, on the one hand, and the centrifugal acceleration is summable to the repulsive gravity acceleration, on the other hand (as it is said above), but it also happens that the two opposite gravitational accelerations, so strengthened by the increase due to the other two accelerations (centripetal and centrifugal), don't cancel each other out, but are both summable with respect to the perihelion precession shift.

In fact, as will be shown shortly, the orbital perihelion precession depends on the strange sum of the four mentioned accelerations (attractive gravity, repulsive gravity, centripetal and centrifugal: "strange" because these forces should cancel each other out, by being opposed).

So let's see if the assumption from which the theory proposed here moves is true, namely if it's true that in perihelion, and only in it, the algebraic sum of the four accelerations (centripetal, centrifugal, attractive gravity and repulsive gravity) influences the perihelion precession motion.

In this regard it should be remembered that, by we having shown before that each point of the planetary orbit is characterized by the acceleration law of the harmonic oscillator pendulum $g=\omega^{2} x$ (where $\omega$ is the angular velocity of the specific point, to be calculated in the manner illustrated above), I assumed that the aforementioned acceleration law, only with regard to the effects of precession perihelion motion, was equal to:

$$
4 g_{(\text {perihelion })}=\dot{\omega}^{2} x
$$

where:

- $g_{(\text {perihelion })}$ is gravity acceleration due to the solar attraction in perihelion;

- $\dot{\omega}=$ is the precession angular velocity;

- $x$ is planet position, that is, its projection on the abscissas axis of the harmonic oscillator, which in the perihelion coincides with the maximum oscillation, i.e. with the radius, therefore with the semi-major axis $a$ (since we have approximated the planetary orbits at the circumferences of radius equal to said semi-axis). 
Before $g_{\text {(perihelion) }}$ there is 4 because we have assumed that, in perihelion, the four accelerations (of attractive gravity, of repulsive gravity, centripetal, and centrifugal) are equal and algebraically summable to each other.

From (43) we can obtain the precession angular velocity:

$$
\begin{gathered}
4 g_{(\text {perihelion })}=\dot{\omega}^{2} a \\
\dot{\omega}=\sqrt{\frac{4 g_{(\text {perihelion })}}{a}} \\
\dot{\omega}=2 \sqrt{\frac{g_{(\text {perihelion })}}{a}}
\end{gathered}
$$

- Calculation of Mercury's perihelion precession velocity

Let us now compute, by means of (44), the Mercury's perihelion precession velocity which, net of the gravitational perturbations due to the other planets and net of other causes incident however on the precession in question, is equal, as it's known, to $43.1 \pm 0.55$ arcseconds/century

First of all, it's necessary to calculate the gravity acceleration that the Sun generates on Mercury in perihelion.

$$
g_{(\text {perihelion })}=\frac{G M}{(\text { perihelion })^{2}}
$$

where:

$G=6.67433 * 10^{-11}$

$M=$ mass of the Sun $\left(1.9891 * 10^{30} \mathrm{~kg}\right)$

Mercury's perihelion $=46,001,046,044$ meters

By replacing in (45) the above-mentioned values, and by knowing Mercury's semi-major axis ( $a$ ) is $57,909,175,678$ meters, we have:

$$
g_{(\text {perihelion })}=0.06273 \mathrm{~m} / \mathrm{s}^{2}
$$

By replacing in (44) the $g$ value found above and the semi-major axis value of Mercury's orbit, we have:

$$
\begin{gathered}
\dot{\omega}=2 \sqrt{\frac{0.06273}{57,909,175,678}} \\
\dot{\omega}=0.000002081584 \mathrm{rad} / \text { year }
\end{gathered}
$$


The result is in $\mathrm{rad} / y e a r$, not in $\mathrm{rad} / \mathrm{s}$, because the perielion shift is a movement due to a condition which arises only in one specific point of the orbit, the perihelion.

By converting the radians in arcseconds/year, we have:

$$
\dot{\omega}=0.4294 \text { arcsec. } / \text { year }
$$

By multiplyng by 100 :

$$
\dot{\omega}=42.94 \text { arcsec./century - Q.E.D. }
$$

At this point it is necessary to make a clarification.

As we have illustrated above, the 4 accelerations (centrifugal, centripetal, of attractive gravity and repulsive gravity) are algebraically joinable together only at the moment when the planet occupies the perihelion.

It is therefore an instantaneous condition, which nevertheless influences the whole planetary orbit with the perihelion precession phenomenon.

By being an instantaneous situation, which lasts for an indefinitely small time, there is the problem to understand how to relate the precession speed with the time, by being an instant indefinitely smaller than a second.

Now, by we having obtained in (44) the measurement of the angular velocity in radians per second (because $g_{\text {perihelion }}$ is measured in $\mathrm{m} / \mathrm{s}^{2}$ ), and since the "second" being a fraction of a terrestrial "year", it's necessary to investigate whether it is possible to relate the "instant" to the "second".

To this end we observe that the instant is not quantifiable, by having a merely conceptual value, as the point for spatial quantities.

A temporal interval, in fact, is composed of an infinite number of instants, similarly, a segment is composed of an infinite number of points.

It is not possible, therefore, to estimate how many times the instant is smaller than the second, as it is not possible to say how many times the point is smaller than the centimeter.

Therefore, if we want to adapt a speed measured in seconds of terrestrial year to a movement that takes place in a single point of the orbit and for a single moment, like the perihelion precession motion, we need to adjust the time scale used to measure the speed in question at the concept of "instant", and, since the fundamental temporal unit that we used in the measurement scale is the year 
(because the second is a fraction of the terrestrial year), the body's speed can only be compared to the terrestrial year.

We can therefore say that at the moment when Mercury occupies perihelion, the planet's perihelion shift velocity is 0.4294 arcseconds/year.

Multiplying by 100 we will have:

$$
\dot{\omega}=42.94 \text { arcsecond/century }
$$

which is precisely the perihelion precession velocity of Mercury.

We report below the table of perihelion precession velocities of all the planets obtained from (44), and compare them with the values obtained by the theory of relativity.

\begin{tabular}{|c|c|c|c|c|}
\hline Planet & $\begin{array}{l}\text { Precession } \\
\text { observed by } \\
\text { Newcomb }\end{array}$ & $\begin{array}{c}\text { Precession } \\
\text { predicted by } \\
\text { R. } \\
\text { Napolitano's } \\
\text { theory }\end{array}$ & $\begin{array}{l}\text { Precession } \\
\text { predicted by } \\
\text { Einstein's } \\
\text { theory }\end{array}$ & $\begin{array}{c}\text { Precession } \\
\text { observed after } \\
\text { Newcomb }\end{array}$ \\
\hline Mercury & $43.4 \pm 2.1$ & 42.94 & 42.98 & $43.1 \pm 0.55$ \\
\hline Venus & $10.6 \pm 36.5$ & 13.44 & 8.61 & $8.4 \pm 4.8$ \\
\hline Earth & $6.9 \pm 7.8$ & 8.35 & 3.84 & $5 \pm 1.2$ \\
\hline Mars & $9 \pm 3.8$ & 4.81 & 1.35 & 8 \\
\hline Jupiter & ------- & 0.7 & 0.0623 & ------- \\
\hline Saturn & ------- & 0.293 & 0.0137 & ------- \\
\hline Uranus & ------- & 0.1023 & 0.0024 & ------- \\
\hline Neptune & ------- & 0.05024 & 0.0008 & $\begin{array}{l}------ \\
\end{array}$ \\
\hline Pluto & ------- & 0.00412 & 0.0000005 & ------- \\
\hline
\end{tabular}

* The values are in arcseconds/century

As it can be seen from the table above, the two theories are substantially equivalent for the inner planets.

As for the outer planets (i.e. Jupiter, Saturn, Uranus, Neptune and Pluto) the two theories diverge sharply: therefore one of the two theories is wrong. 
It is up to the astronomers the final judgment on which of the two theories is the correct one, not easy judgment, because of the very small quantity of observational studies on the perihelion precession of outer planets.

\section{- $\quad 5.2$ - Gravity force: exception to the first principle of dynamics}

But how is it possible that, when the planet is in perihelion, where the attractive force is aligned with the repulsive one, these two forces do not cancel each other out, but give life to the perihelion precession shift?

It's a mystery that, in my opinion, can be explained in only one way, that is, believing that the clash between the attractive and repulsive gravitational force gives rise to a particular type of gravitational force, and consequently of gravitational potential energy — that we'll define strengthened force $\left(\frac{4 G M m}{R^{2}}\right)$ and strengthened energy $\left(\frac{4 G M m}{R}\right)$ - arising from the forced non-birth, in the perihelion, of the $F_{r x}$ force, which prevents the planet from occupying the perihelion it had previously occupied, by forcing it to "cut" the angle more towards the inside and preventing the elliptical orbit from closing itself, thus giving life to that particular flower-shaped orbit, which we see in the image on next page: 


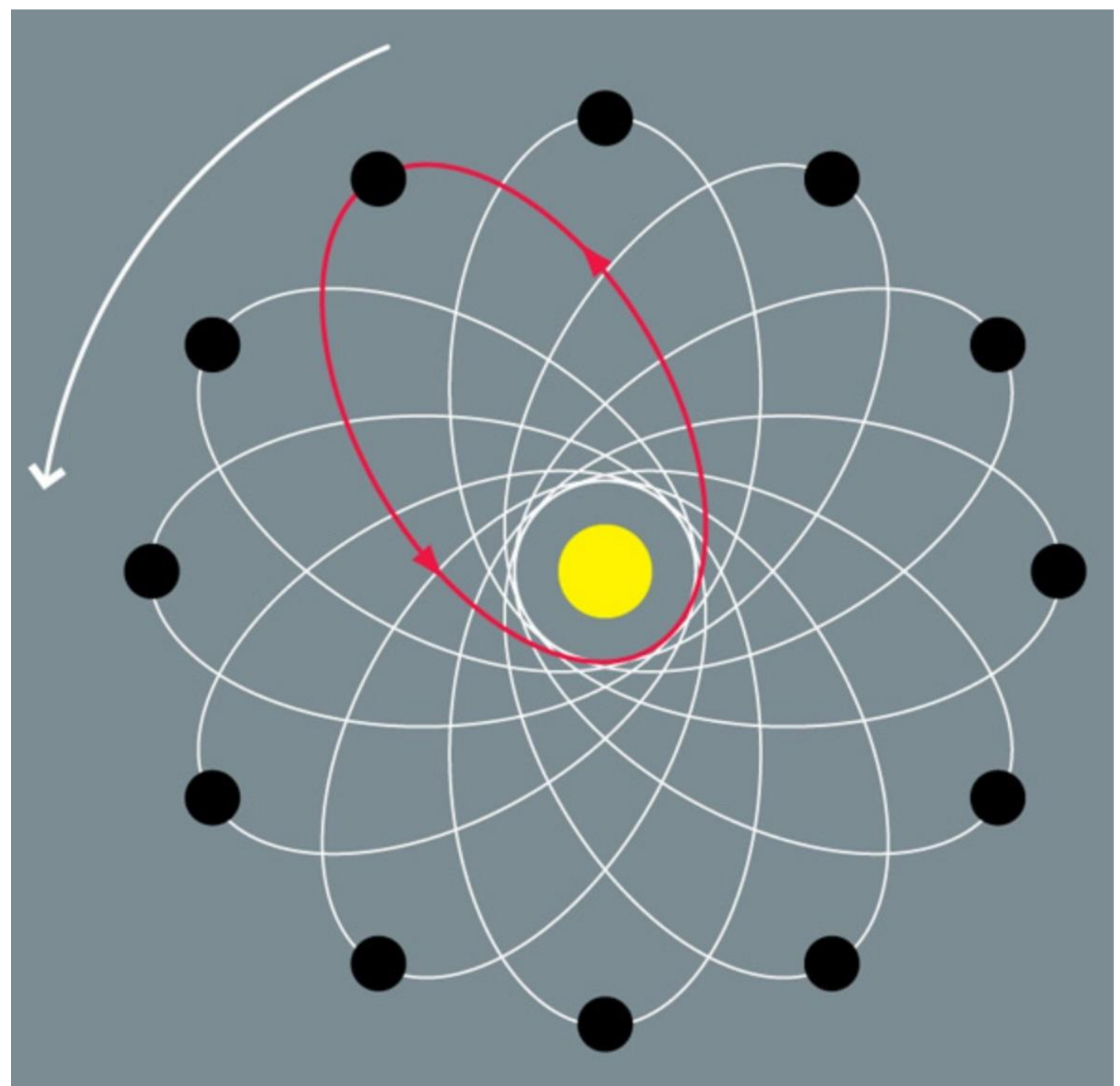

The perihelion precession motion, in essence, derives from the fact that, in the aforesaid point, the energy arising from the forced non-birth of $F_{r x}$ force cannot find another outlet except in the aforementioned motion, so this energy is channeled in the only possible way, i.e. by causing an impediment for the planet to reoccupy the previous perihelion, which ultimately produces the shift movement of the apsis line.

This confirms the absolute singularity of the gravitational force, which, considering the mathematical findings obtained here, demonstrates that it does not succumb to the first principle of dynamics, according to which two opposing forces 
cancel each other, but that it gives rise, whenever these two forces derive from a single body (the central one), to two movements around it: the revolution of the bodies around the attractor-repeller, and periapsis precession motion, i.e. the orbital point closest to the central body.

So, there would be two exceptions to the first principle of Newton's dynamics that seem to occur in celestial mechanics, because even in the points other than perihelion the two opposing gravitational forces do not cancel out, but generate the revolution motion, thanks to the $F_{r x}$ force arising from the different pressure (or energy) values existing in the attractio-repulsio zone.

The trajectory described by the perihelion precession motion is circular because, in the aforementioned point, the alignment of the attractive and repulsive gravity forces gives rise to a strengthened centripetal force, i.e. generating like a wall that stems the movement of the planet, preventing it from re-occupying the specific point in which this energy arises.

In other words the trajectory described by the perihelion precession motion is circular, because such is the trajectory described by a body launched at a certain speed with a tangential direction and subjected only to a constraint which generates centripetal acceleration (as the example of the ball tied to a rope seen above).

This strengthened energy arising only in perihelion would have this formula:

$$
U=m * 4 g * R
$$

As we notice, this energy differs from the potential energy of exclusively attractive field for the presence of 4 before $g$.

\section{- $\quad 5.3$ - The deflection of stars-light behind the Sun}

As it's known, one of the proofs of relativity is the fact that from the Earth it is possible to see the light emitted by stars positioned behind the Sun, which we should not therefore see.

The proof consists first of all in the fact that the light is considered not to have a mass, so that the circumstance that is deviated near the Sun could not depend by gravity force exerted by the star, but by the fact that the space around the Sun is curved by its mass, and consequently the light of the star behind the Sun 
cannot help but be deflected and be visible to us on Earth.

Another presumed proof of the validity of relativity is, secondly, the measurement of the deflection angle, which is twice that of the Newtonian theory, but in harmony with relativity theory.

Well, by the result we have reached in the eq. (44), it is easy to explain why the evidence shows that the deflection angle of light is twice the one predicted by Newton's theory.

In this regard it should be noted that the hyperbolic orbit described by the ray of light emitted by the star behind the Sun is subjected, at the point where the deflection occurs (which is the one closest to the Sun), to the same characteristics as the perihelion of elliptical orbits, i.e. the equality among the 4 accelerations (attractive gravity, centripetal, repulsive gravity and centrifugal), as well as the fact that they are all aligned with each other (albeit with the first two opposite to the second two), from which we have their summability with regard to perihelion precession which, as shown above, justifies the 4 before $g$ in the acceleration law of the harmonic oscillator $g=\omega^{2} \mathrm{x}$, which then becomes, with respect to the perihelion precession motion:

$$
4 g_{(\text {perihelion })}=\dot{\omega}^{2} a \rightarrow \dot{\omega}=2 \sqrt{\frac{g_{(\text {perihelion })}}{a}}
$$

Consequently, at the point where the deflection of light occurs, it is subjected, similarly to the perihelion of planetary orbits, to the precession motion, which takes place with an angular velocity equal to twice the angular velocity of the revolution motion of the planets around the Sun.

In fact, as we have seen above, the acceleration law to which the planets are subjected coincides perfectly with the one of the harmonic oscillator, i.e. $g=\omega^{2} x$, which in perihelion becomes $\omega=\sqrt{\frac{g_{(\text {perihelion })}}{a}}$, which is exactly half the value of the angular precession velocity we have reached in (44), and this is the reason why the deflection angle that the light undergoes at the point closest to the Sun is twice what is obtained from Newton's theory.

Basically, light is also subjected to gravity, like any macroscopic body, and even subjected to precession motion, like planets and like all bodies with mass, and 
precession was not taken into account in the calculation of the angle deflection by Newton's theory.

In other words, the trajectory described by light is not the one that should be according to the Newtonian theory, because the point closest to the Sun "precedes" with respect to the one predicted by the aforementioned theory, because, due to the strengthened energy which is generated at the point where the deflection occurs, the light is forced to follow a more internal path in such a way as to cut the angle, similarly to what happens for the precession of the perihelion of the planetary orbits, so that its angular velocity will increase in twice the size of the Newtonian theory, and therefore the deflection angle of the light can only be twice as much as expected.

After all, this is in harmony with the so-called "dual" nature of the photon, which is believed to be a corpuscle and a wave at same time.

If, therefore, the photon is "also" a corpuscle, there is no strangeness in the fact that it is affected by gravitational attraction in the same way as any other massive body, thus also undergoing the precession motion when it passes at the point closest to the attractor-repulsor.

\section{- 5.4 - The black hole radius}

A further confirmation of the fact that the light, like every body with mass, is subjected to the precession movement is the applicability of the perihelion precession formula we have obtained in (44) also to black holes, as will be shown in below.

The black hole, as it's known, has the particularity of being able to trap in its gravitational field any object ends up in its range of action, including light.

Let's see if it is possible to calculate by the (44) the radius of the black hole, that is the limit beyond which nothing, once entered, can escape it.

Assuming that the cause of the impossibility for light to come out of the black hole is the same that prevents the planets from occupying the previous perihelion, causing its precession - that is, a particular type of strengthenedenergy arising from the alignment between the attractive gravity force and repulsive gravity force (due to the non-arising of the force $F_{r x}$ ), that forces the body 
to "cut" the angle more inward — and that this alignment of forces occurs not in a single point, like instead occurs in the case of the perihelion of planetary orbit, but along the entire circumference delimiting the black hole, we will have that the angular velocity of light around the black hole due to the "continuous" precession to which it is subjected by the aforementioned energy, will be equal to:

$$
\dot{\omega}=2 \sqrt{\frac{g}{R}}
$$

where $R$ is the radius of the black hole which, by having a circular structure, dispenses us from speaking of periapsis and semi-major axis, and this allows us to consider the radius both in the numerator (for the calculation of $g$ ) and in the denominator, so $g$ will be equal to:

$$
g=\frac{G M}{R^{2}}
$$

and (46) becomes:

$$
\begin{gathered}
\dot{\omega}=2 \sqrt{\frac{\frac{G M}{R^{2}}}{R}} \\
\rightarrow \dot{\omega}=2 \sqrt{\frac{G M}{R^{3}}}
\end{gathered}
$$

But, since we hypothesized that the precession is "continuous", that is to say that it operates not in a single point of the trajectory followed by the light around the black hole, but uninterrupted along the whole circumference of the black hole, we can equal the eq. (47) to the angular velocity proper to the circular motion, which is:

$$
\omega=\frac{2 \pi}{T}
$$

Therefore, from the comparison between the equations (47) and (48) we can write:

$$
\begin{gathered}
2 \sqrt{\frac{G M}{R^{3}}}=\frac{2 \pi}{T} \\
\rightarrow \frac{1}{2} \sqrt{\frac{R^{3}}{G M}}=\frac{T}{2 \pi}
\end{gathered}
$$

Multiplying both sides by $2 \pi$, we have: 


$$
\pi \sqrt{\frac{R^{3}}{G M}}=\mathrm{T}
$$

By squaring both sides:

$$
\begin{array}{r}
\pi^{2} \frac{R^{3}}{G M}=T^{2} \\
\rightarrow \quad R^{3}=\frac{T^{2} G M}{\pi^{2}}
\end{array}
$$

But we also know that, in circular motion, $T$ is equal to:

$$
T=\frac{2 \pi R}{V}
$$

and that in our case $V$ can be replaced with the speed of light $c$, because we are verifying the applicability of the eq. (44) to a black hole which, as is known, is capable of blocking the light too, so we will have:

$$
T=\frac{2 \pi R}{c}
$$

by replacing the aforementioned value of $T$ in the eq. (49), we will have:

$$
\begin{gathered}
R^{3}=\left(\frac{2 \pi R}{c}\right)^{2} \frac{G M}{\pi^{2}} \\
\rightarrow \quad R^{3}=\frac{4 \pi^{2} R^{2}}{c^{2}} \frac{G M}{\pi^{2}} \\
\rightarrow \quad R^{3}=\frac{4 R^{2} G M}{c^{2}}
\end{gathered}
$$

by dividing both sides by $R^{2}$, we'll have:

$$
R=\frac{4 G M}{c^{2}}
$$

This equation expresses the value of the distance from the center of the black hole within which everything, including the light, once entered, no longer comes out.

Let's now try to verify concretely the efficacy of the eq. (50) using it to calculate the radius of the black hole Sagittarius $A^{*}$ located at the center of the Milky Way, taking as its mass the average one resulting from the different estimates made by the astronomers, that is 3.75 million times the mass of the Sun.

$$
\begin{aligned}
& M_{\text {Sagittarius } A}=3.75 * 1,000,000 \text { mass of Sun }=7.35967 * 10^{36} \mathrm{~kg} \\
& \mathrm{G}=6.67433 * 10^{-11} \\
& c^{2}=89,875,517,873,681,764
\end{aligned}
$$

By replacing in (50) the above-mentioned values, we have: 


$$
\begin{gathered}
\mathrm{R}=\frac{4 * 6.67433 * 10^{-11} *\left(7.35967 * 10^{36}\right)}{89,875,517,873,681,764} \\
R=22.157 .162 \mathrm{~km}
\end{gathered}
$$

As you can see the result to which (50) leads, equal to about 22 million Km, is in good agreement with the observational estimates, which quantify the radius of Sagittarius $A$ * in about 22 million $\mathrm{km}$.

We can therefore conclude that, with good probability, the black hole generates, at least at a distance equal to the value obtainable from the eq. (50), a gravitational strengthened energy that forces the particles moving in there to orbit in perpetuity around the black hole center, like we will better see later.

It's worth to notice that the Schwarzschild's radius is notoriously equal to $R=\frac{2 G M}{c^{2}}$, that is the half of the radius we have reached in (50), so that not only it doesn't correspond to the radius of Sagittarius $A *$ resulting from the observations.

\section{- $\quad 5.5$ - The nuclear radius: the gravitoelectrostatic force}

Let's see now if it is possible, by using our theory, to obtain the atomic nuclear radius.

We know from Einstein that the energy contained in the atomic nucleus by the very fact of having a mass, which is defined the total mass-energy", is equal to $E_{m}=M c^{2}$, where $M$ is the mass of the nucleus.

Let us suppose that $M c^{2}=2 * E_{k}$, where $E_{k}$ is the kinetic energy of nucleons.

Now, let's suppose that within the nucleus a pendulum harmonic oscillator there exists, and that the following law, inferred by induction in reference [20], be applicable:

$$
-\langle\phi\rangle=2 *\left\langle E_{k}\right\rangle
$$

where $\phi$ be the gravitoelectric potential energy of nucleons $U_{g e}$, that we will derive shortly, then:

$$
-U_{g e}=M c^{2}
$$

As it's known, the gravitational potential energy of a body subjected to the attractive gravitational force is: 


$$
U=-F_{g} * R
$$

where $F_{g}$ is gravity force which, by virtue of the second principle of dynamics, can also be written as follows:

$$
F_{g}=m * g
$$

Therefore the (52-bis) becomes:

$$
U=-m * g * R
$$

Where $m$ is the test mass, $g$ is the gravity acceleration and $R$ is the distance of the test body from the center of the attractor body (for instance the Earth).

Let us suppose that in the atomic nucleus the force of gravity merge with the electrostatic force, giving life to the gravitoelectrostatic force:

$$
F_{g e s}=\frac{4 G K M M}{\pi R^{4}}
$$

The term $\pi$ appears because we have supposed that in the nucleus a pendulum harmonic oscillator there exists, giving rise to a circular trajectory.

Then, by applying eq. (52-bis), the gravitoelectric potential energy $U_{g e}$ becomes:

$$
U_{g e}=-F_{g e s} * R
$$

Therefore:

$$
\begin{gathered}
U_{g e}=-\frac{4 G K M^{2}}{\pi R^{4}} * R \\
U_{g e}=-\frac{4 G K M^{2}}{\pi R^{3}}
\end{gathered}
$$

Now we replace in (52) the value of $U_{g e}$ just found, and we'll have:

$$
\begin{aligned}
& \frac{4 G K M^{2}}{\pi R^{3}}=M c^{2} \\
& R=\sqrt[3]{\frac{4 K G M}{\pi c^{2}}}
\end{aligned}
$$

It's worth to specify that the eq. (57) doesn't express the nuclear radius, but the full nuclear radius, i.e. the radius of the sphere containing all the nuclear matter, like we'll better explain later.

Let's now examine the concrete efficacy of (57), trying to calculate the nuclear radius of different atoms (chosen randomly in the periodic table of elements), and compare it to the radius observed by nuclear physicists. 
Let's start with the gold atom.

It's opportune to state that the formula that physicists have deduced experimentally for the determination of the nuclear radius (mostly of the medium and heavy stable nuclei) is the following:

$$
R=r_{0} * \sqrt[3]{A} * 10^{-15} \text { meters }
$$

Where $r_{0}$ is equal to 1.21 (see reference [15]), and $A$ is the mass number, i.e. the sum of protons and neutrons (it is indicated at the top left next to the element symbol in the periodic table of the elements).

It should be noted that the eq. (58) is obtained by the electron scattering experiments (see reference [15]), i.e. from the angular distribution (diffraction) of the incident beam of particles fired on a target nucleus. Now, we'll see below that, by dividing by 2 the right-hand side of the eq. (57) we'll have just the value of the empirical nuclear radius given by the eq. (58)

$$
R=\frac{1}{2} * \sqrt[3]{\frac{4 K G M}{\pi c^{2}}}
$$

Let's see if the eq. (59) is true.

Well, the mass number of the gold atom is:

$$
A=197
$$

so the "experimental" radius deriving from (58) is:

$$
\begin{gathered}
R=1.21 * \sqrt[3]{197} * 10^{-15} \\
R=7.0405 * 10^{-15} \text { meters }
\end{gathered}
$$

Let's now calculate the nuclear radius of the aforesaid atom by means of (59), where:

$M$ is the mass of nucleus, i.e. the sum between the protons mass and the neutrons mass $=(Z * 1.6726) 10^{-27} \mathrm{~kg}+(N * 1.6749) 10^{-27} \mathrm{~kg}$

$K$ is Coulomb's constant $=8.99 * 10^{9}$

$G$ is gravitational constant $=6.67433 * 10^{-11}$

$\pi=3.1415$

$c=$ speed of light squared $(299,792,458)$

For convenience we quote below the eq. (59) and replace in it the values, written above, of $M, G, K, \pi$, and $c$. 


$$
\begin{gathered}
R=\frac{1}{2} \sqrt[3]{\frac{4 K G M}{\pi c^{2}}} \\
R=\frac{1}{2} * \sqrt[3]{\frac{4 *\left(8.99 * 10^{9}\right) *\left(6.67433 * 10^{-11}\right) *[(79 * 1.6726)+(118 * 1.6749)] * 10^{-27}}{3.1415 * 89875517873681764}} \\
R=7.0500 * 10^{-15} \text { meters }
\end{gathered}
$$

We now compare the theoretical value of the nuclear radius we have obtained in (61) and the value of the radius obtained by the empirical formula (60):

$$
\frac{7.0500 * 10^{-15}}{7.0405 * 10^{-15}}=1.001
$$

As you can see, the margin of error is practically non-existent.

Let us now apply (59) to different atoms randomly chosen from the periodic table of elements (we choose only some of them for convenience), with $r_{0}=1.21 \mathrm{fm}$.

\begin{tabular}{|c|c|c|}
\hline Atom & $\begin{array}{c}\text { Nuclear radius empirically } \\
\text { observed } \\
\left(R=1.21 * 10^{-15} * \sqrt[3]{A}\right. \\
\text { meters })\end{array}$ & $\begin{array}{c}\text { Nuclear radius predicted } \\
\text { by R. Napolitano's } \\
\text { theory }\end{array}$ \\
\hline Silver & $5.7444 * 10^{-15}$ & $\begin{array}{c}1 \\
2\end{array} \sqrt[3]{\frac{4 K G M}{\pi c^{2}}}$ meters $)$ \\
\hline Gallium & $4.9628 * 10^{-15}$ & $4.9694 * 10^{-15}$ \\
\hline Mercury & $7.0996 * 10^{-15}$ & $7.1092 * 10^{-15}$ \\
\hline Iron & $4.6292 * 10^{-15}$ & $4.6353 * 10^{-15}$ \\
\hline Bromum & $5.1919 * 10^{-15}$ & $5.1988 * 10^{-15}$ \\
\hline Lead & $7.1692 * 10^{-15}$ & $7.1789 * 10^{-15}$ \\
\hline Iridium & $6.9925 * 10^{-15}$ & $7.0020 * 10^{-15}$ \\
\hline
\end{tabular}

The above table fully confirms the correctness of the formula (59).

It's worth to specify that the eq. (57) gives the full nuclear matter radius, which is twice the nuclear radius resulting from the scattering phenomenon. This is due to the fact that, like we'll see in the paragraph 5.11, the scattering of incident beam allows to detect only half the full size of the nucleus, because one has to take into account also the shadow nucleons orbiting around the real ones, and indirectly resulting from the nuclear reactions, i.e. the absorption of the alpha particles fired on 
the nucleus which occurs beyond the nuclear radius given by the eq. (58).

For now, the above calculations are sufficient to state that gravity, whatever its cause, affects not only the macroscopic world, but also the microscopic one, albeit with the particularity of merging with the electrostatic force.

Moreover, the joint action of the two forces is completely consistent with the so-called "dual" nature of the fundamental particles: the corpuscular aspect of the nucleons (protons and neutrons) creates gravitational force, the undulatory aspect of nucleons originates electrostatic force.

Would the formula (57) be the proof of the famous "quantum gravity"?

Beyond the terminological questions, we reduce as much as we want the persuasiveness of the reasoning that led us to the eq. (57), we cannot deny the existence of the gravity force even at the quantum level, at least at the nuclear level. The aforementioned formula, in fact, is clear: at the level of the atomic nucleus, the gravity force and the electrostatic force merge together to give life to an energy, expressed by the eq. (55), which allows to contain the energy inherent in the mass itself $\left(E=m c^{2}\right)$.

The strengthened nature of the energy expressed in the eq. (55) is confirmed by the derivative character of this formula with respect to the orbital periapsis precession formula (43) and to the black hole radius formula (50), where we have the same kind of energetic nature (see the presence of number 4 in all the formulae).

- $\quad 5.6$ - The non-decreasing velocity of galaxy stars: dark matter crisis

As it's known, the stars orbit the black hole at almost constant orbital velocity, unlike the Keplerian system (like our solar system), in which orbital velocity of celestial bodies decreases with the increase of distance from the Sun.

In truth, the stars of the galaxies behave according to the Keplerian system (and therefore with the Newtonian mechanics), only in the proximity zone, the one closest to the black hole, where the stars motion respects the Newtonian gravitational model, in which gravity force is inversely proportional to the square of the distance from the attractor, and potential energy is directly proportional to the distance.

Beyond that proximity zone, the orbital velocity of the stars remains substantially constant, until the end of the galactic disk, where it returns to increase. 
The science explains the stability of the stars orbit located in the intermediate zone (which, we repeat, they move at constant speed) hypothesizing the existence of dark matter (not visible) inside the galaxies, which "would help" the gravity force exerted by the black hole to keep the stars within the range of the hole itself, thus preventing the stars from moving away from it permanently, despite of their high speed with respect to the gravity generated by the mass of the black hole.

This dark matter would have the characteristic of not being observable, although it exerts gravitational force and represents about $75 \%$, or perhaps even more, of the overall mass that makes up the universe.

However, despite of the considerable efforts made by researchers both in the nuclear and in the astronomy physics, it has not yet been possible to find direct evidence of dark matter.

Below we propose a possible explanation of the strange phenomenon of the constancy of the orbital velocity of the stars, hypothesizing the existence of an attractive-repulsive gravitoelectric forces-field that would arise starting from a certain distance from the black hole, i.e. beyond the zone where instead the attractive-repulsive gravitational (not gravitoelectric) field would operate.

From the strange non-observability of the black hole, it has been hypothesized that it was composed only of protons (without therefore neutrons and electrons), not joined together to form a single body, due to the repulsive force among them that would preclude this.

Furthermore, we have hypothesized that the galactic system could be compared to the atom system, where the black hole would perform the same functions as the atomic nucleus, and the stars orbiting the black hole would be the electrons orbiting the nucleus.

If this were true, the equation $E=M c^{2}$, which expresses the energy possessed by the nucleus by the very fact of having a mass, should undergo a modification with regard to the galactic nucleus (the black hole).

If the black hole nucleus is only composed of protons, the consequence could be that the energy it possesses will be directly proportional not to its mass, but to its charge $\mathrm{Q}$, since the protons have an electric charge, so it is reasonable to assume that the Einsteinian formula seen above becomes: 


$$
E=Q c^{2}
$$

Let's see if (62) is true.

By assuming that the black hole exerts an attractive-repulsive gravitoelectric field from a certain distance from it, responsible for the revolution of the stars around it, it follows that the gravitoelectric revolution-energy possessed by the aforementioned stars will be equal to the right-hand side of (53), without 4 , because it is a not strengthened force:

$$
E_{r}=\frac{G K M m}{R^{4} \pi} * R
$$

Where $m$ is the total mass of the visible stars of the Milky Way, so without the hypothetical dark matter.

Now, if it is true that the energy expressed by (62) is responsible for the revolution of the stars around the black hole, it must be equal to the gravitoelectric revolution-energy seen in (63), therefore, from the comparison of the (63) and (62) we can write:

$$
\frac{G K M m}{R^{3} \pi}=Q c^{2}
$$

So eq. (64) becomes:

$$
\frac{G M}{R} * \frac{K m}{R^{2} \pi}=Q c^{2}
$$

But from Newtonian mechanics, to which the mechanics proposed here are inspired, we also know that:

$$
v=\sqrt{\frac{G M}{R}} \rightarrow v^{2}=\frac{G M}{R}
$$

by replacing in eq. (65) $\frac{G M}{R}$ with $v^{2}$, we'll have:

$$
\begin{aligned}
& v^{2} * \frac{m K}{R^{2} \pi}=Q c^{2} \\
& v^{2}=\frac{Q c^{2} R^{2} \pi}{m K} \\
& v=c R \sqrt{\frac{Q \pi}{m K}}
\end{aligned}
$$

Since the velocity of the stars does not decrease with the increase of distance from the black hole, we can hypothesize that the attractive-repulsive gravitoelectric field generated by the protons does not depend on the distance, so we 
can impose as a condition that the radius be undimensional and be equal to $\mathrm{R}=1$, and consequently we can take as undimensional the other non-constant quantity of the right-hand side of eq. (66), $\frac{Q}{m K}$, then the eq. (66) becomes:

$$
\rightarrow v=c \sqrt{\frac{Q \pi}{m K}}
$$

with $\frac{Q}{m K}$ taken as undimensional.

Now let's try to calculate, by using the eq. (67), the speed of the stars of the Milky Way (our galaxy), which (excluding those closest to the black hole, to which the Newtonian formula $v=\sqrt{\frac{G M}{R}}$ is applicable) orbit the central black hole Sagittarius $A *$ at an average orbital velocity, almost constant, estimated between 220 and $240 \mathrm{~km} / \mathrm{sec}$.

First of all, it is necessary to determine the electric charge $Q$ of Sagittarius $A^{*}$.

To this end it is necessary to determine the number of protons making up Sagittarius $A^{*}$, and then multiply it by the electric charge of the single proton.

To quantify the number of protons located inside Sagittarius $A *$ it's enough to divide the mass of the latter by the mass of the single proton.

Mass of Sagittarius $A^{*}=3.75 * 1,000,000 M_{\text {Sun }}=3.75 * 1,000,000 *$ $1.9891 * 10^{30} \mathrm{~kg}$

Mass of proton $=1.6726 * 10^{-27} \mathrm{~kg}$

Number of protons making up Sag. $A^{*}=\frac{3.75 * 1,000,000 * 1.9891 * 10^{30}}{1.6726 * 10^{-27}}=$

$=4,4595988281717087169675953605166 * 10^{63}$

To obtain the electric charge $Q$ of Sagittarius $A^{*}$ we multiply the number of protons obtained in (68) by the charge of the single proton, which is equal to $1.621 * 10^{-19}$ Coulomb, so we'll have:

$$
\begin{aligned}
& Q=\left(4.4595988281717087169675953605166 * 10^{63}\right) *\left(1.621 * 10^{-19}\right) \\
& Q=7.2290097004663398302044720793973 * 10^{44} \text { Coulomb }
\end{aligned}
$$

Let us now determine the value $m$ of the total mass of the stars of the Milky Way. 
According to astronomers, it ranges from a minimum of 200 to a maximum of 350 billion solar masses $\left(1.9891 * 10^{30} \mathrm{~kg}\right)$.

Applying the minimum estimate (200 billion solar masses), $m$ will be equal to:

$$
m=200 * 10^{9} *\left(1.9891 * 10^{30}\right)=3.9782 * 10^{41} \mathrm{~kg}
$$

We now replace in (67) the values of $Q$ and $m$ found respectively in (69) and in (70), and also those, already known, of $c, \pi$ e $K$ :

$$
\begin{aligned}
v=299,792,458 * \sqrt{\frac{\left(7.2290097004663398302044720793973 * 10^{44}\right) * 3.1415}{\left(3.9782 * 10^{41}\right) *\left(8.99 * 10^{9}\right)}} \\
v=238,894 \mathrm{~m} / \mathrm{sec} \\
v=238.894 \mathrm{Km} / \mathrm{sec}
\end{aligned}
$$

As we can note, the stars orbital velocity of the Milky Way predicted by (67) is perfectly in accordance with the observation, whose estimates range, we repeat, from 220 to $240 \mathrm{~km} / \mathrm{sec}$.

We have thus shown that the stars orbital velocity is directly proportional to the electric charge of the black hole, and inversely proportional to the total mass of the visible stars that make up the galaxy, without considering the contribution of dark matter, whose real existence seems to be no more so certain.

\section{- 5.7 - Black holes: "proton stars"}

According to the mathematical findings obtained here, it is very probable that the black hole is not what remains of the gravitational collapse of a star, as current opinion holds, but is a concentration of protons, which can lead us to coin, in their regards, the definition of "proton stars".

Such protons would not be joined together to form a single massive body (which could justify their non-observability), by remaining completely autonomous particles among which would operate electrostatic repulsion, even though opposed by the gravitational strengthened-energy generated by the central shadow protons, which forces these latter to remain confined within the radius given by (50).

In summary, the central shadow protons would give rise to two types of gravitational energy:

1) gravitational strengthened-energy (arising from the collision between 
the attractive and repulsive gravitational forces generated by the same protons, due to the forced non-arising of the force $F_{r x}$ ), which would oppose the movement of protons (as we'll say better later), confining them, we repeat, within a sphere of radius equal to the value expressed by $(50)$;

2) gravitational revolution-energy, which would exist in the proximity zone (the one closest to the black hole), which would cause the revolution of the stars around the black hole according to the KeplerianNewtonian model, i.e. with decreasing speed as the distance from the center increases $\left(v=\sqrt{\frac{G M}{R}}\right)$.

Starting from a certain distance from the black hole, the attractive-repulsive gravitational field would turn into an attractive-repulsive gravitoelectric field, generating gravitoelectric revolution-energy, responsible for the revolution at almost constant speed of the stars around the central black nucleus.

Anyway, the change of the modus-operandi of the gravitational force according to the spatial zone observed, would not be a novelty, by we having already found this peculiarity in the examination of the solar system, in which there is similarly a proximity zone where gravity is only attractive, and a farther one, where gravity changes its effect to become attractive and repulsive at the same time.

This is all I can say about the black holes at this point of the present work, but I will make it clearer in the next paragraphs $(5.10,5.11$ and 5.12).

\section{- 5.8 - The orbital speed and the orbital radius of electrons}

If it is true that the atom can be compared to a galaxy — but we already had a confirmation demonstrating the full correctness of the equation $E=Q c^{2}$ seen in (62), which, referring to the protons of the black hole, shows that they behaves, with respect to the galaxy, in the same way as the nucleus of the atom behaves with respect to the atom itself, in which the similar equation $E=M c^{2}$ applies - we try to determine the orbital velocity of the electrons around the nucleus, using the same logical-mathematical process seen above for the orbital velocity of the stars, even though we don't have a sure parameter as a reference, since the electron velocity 
having never been experimentally measured.

By assuming, analogously to what has been done for the stars of galaxies, that nuclear energy is responsible for the revolution of the electrons around the nucleus, and that therefore this exerts an attractive-repulsive gravitoelectric field starting from a certain distance from it, it would follow, as a logical consequence, the comparison between the total mass-energy equation $E=M c^{2}$ and the gravitoelectric revolution-energy equation that we have seen in (63), so we will have:

$$
\frac{G K M m}{R^{4} \pi} * R=M c^{2}
$$

where $m$ is the total mass of the electrons, $M$ is the mass of the nucleus, and $g_{e}$ is the gravitoelectric acceleration generated by the nucleus on the electrons, so eq. (71) becomes:

$$
\frac{G M}{R} * \frac{m K}{R^{2} \pi}=M c^{2}
$$

From Newtonian mechanics we know that $v=\sqrt{\frac{G M}{R}}$, so $v^{2}=\frac{G M}{R}$.

By replacing in the (72) $\frac{G M}{R}$ with $v^{2}$, we'll have:

$$
\begin{aligned}
& v^{2} * \frac{m K}{R^{2} \pi}=M c^{2} \\
\rightarrow & v^{2}=\frac{M c^{2} R^{2} \pi}{m K} \\
\rightarrow & v=c R \sqrt{\frac{M \pi}{m K}}
\end{aligned}
$$

By assuming that the velocity of the electrons does not decrease as the distance from the nucleus increases, analogously to what happens for the stars of the galaxies, we can hypothesize that the attractive-repulsive gravitoelectric field generated by the atomic nucleus does not depend on the distance, so we can impose as a condition that the radius be undimensional and be equal to $\mathrm{R}=1$, and consequently we can take as undimensional the other non-constant quantity of the right-hand side of eq. (74), $\frac{M}{m K}$, then the (74) becomes:

$$
\rightarrow v=c \sqrt{\frac{M \pi}{m K}}
$$

with $\frac{M}{m K}$ taken as undimensional. 
Now let's try to calculate the speed of the electrons of the hydrogen atom, which, according to the theory proposed here, should orbit the nucleus at the speed expressed by (75), where, it is repeated, $m$ is the total mass of the electrons and $M$ is the total mass of the nucleons.

$$
\begin{aligned}
& m_{(\text {electrons } H)}=\text { number of electrons of Hydrogen } *\left(9.11 * 10^{-31}\right)=1 \\
& *\left(9.11 * 10^{-31}\right)=9.11 * 10^{-31} ; \\
& M_{(\text {nucleus } H)}=\text { Mass number } A *\left(1.6726 * 10^{-27}\right)=1 * 1.6726 * 10^{-27}
\end{aligned}
$$

We now replace the above values of $m$ and $M$ in the (75), also those already known of $c, \pi$ and $K$ :

$$
\begin{gathered}
v=299,792,458 \sqrt{\frac{\left(1.6726 * 10^{-27}\right) * 3.1415}{\left(9.11 * 10^{-31}\right) *\left(8.99 * 10^{9}\right)}} \\
v=240,129 \mathrm{~m} / \mathrm{sec} \\
v=240.129 \mathrm{Km} / \mathrm{sec}
\end{gathered}
$$

This is the theoretical speed of electrons orbiting around the hydrogen atomic nucleus: as we can see, the velocity of the electrons obtained in (75-bis) is almost equal to the orbital velocity of the stars of the Milky Way we have reached in (70bis): this further confirms our assumption that the galaxy system is very similar to the atom system.

It is also true that, for atoms with a higher mass number, the velocity of the electrons changes (for example, for the gold atom, the speed of the electrons predicted by (75) is about $382 \mathrm{~km} / \mathrm{sec}$ ), but its value is not too far from the orbital velocity of the stars usually found in galaxies.

Note that the model proposed here differs from the one proposed by Rutherford, who hypothesized that the atom system was similar to the solar system, where the electron was considered to be subjected, according to the Newtonian model, only to a central electrostatic attractive force.

This model was not accepted because it was pointed out that the electron, while rotating around the nucleus, should emit radiations and consequently should lose energy, and then fall on the nucleus.

But in our model, in which the electron is subjected to an attractive-repulsive field, not in the sense conceived by Maxwell, but in the sense of a force-field, there would not be any radiation to emit, and the fall towards the nucleus would be averted 
precisely by the repulsive force.

Let's see if (75) can find a confirmation in experimental data.

From quantum mechanics it turns out that in heavy atoms the lowest electron orbital radius is about 100 times larger than the nuclear radius (in particular see page 121 of reference [2]): let's see if we can demonstrate this by means of our theory.

Let's take the lead atom, notoriously heavy (its mass number $A$ is 208).

Let's calculate by (75) the velocity of electrons of lead atom.

We know that in the lead atom the atomic number is 82 , so, to calculate the total mass of electrons $(m)$ we have to multiply the atomic number 82 by the electron mass $9.11 * 10^{-31} \mathrm{~kg}$ :

$$
m=7.4702 * 10^{-29} \mathrm{~kg}
$$

Now let's replace in (75) the above value of $m$ and the value $M$ of nuclear mass of lead atom $[(82 * 1.6726)+(126 * 1.6749)] * 10^{-27} \mathrm{~kg}$ :

$$
\begin{gathered}
v=299,792,458 \sqrt{\frac{[(82 * 1.6726)+(126 * 1.6749)] * 10^{-27} * 3.1415}{\left(7.4702 * 10^{-29}\right) *\left(8.99 * 10^{9}\right)}} \\
v=382,606 \mathrm{~m} / \mathrm{sec}
\end{gathered}
$$

Now let's calculate the lowest electron orbital radius of lead atom.

For this purpose we can consider a nuclear model in which the nucleus is orbited not by shadow nucleons, but by electrons, so we can use the formula (53) and compare it to twice the kinetic energy of electrons (because, we repeat, this time we assume that the orbiting particles within the nucleus are these latter):

$$
\begin{gathered}
F_{g e s} * R=m v^{2} \\
\frac{m * g_{e s}}{R \pi}=m v^{2}
\end{gathered}
$$

where $m$ is the total mass of electrons of lead atom, $v$ is their velocity indicated in (75-ter), and $g_{e s}$ is the gravitoelectrostatic acceleration $\left(\frac{G K M}{\pi R^{4}}\right)$.

This time we have to use the gravitoelectric revolution potential energy (without 4) because we have assumed that the electrons are not endowed with the speed of light (as we'll see better at the end of paragraph 5.11), so in the (75-quater) we can take as $v$ the value of electron velocity derived by (75), i.e. 382,606 m/sec.

From (75-quater) we have: 


$$
\begin{gathered}
\frac{G M K}{R^{2}} * \frac{m}{R \pi}=m v^{2} \\
\frac{G M K m}{R^{3} \pi}=m v^{2} \\
G M K=R^{3} \pi v^{2} \\
R^{3}=\frac{G M K}{\pi v^{2}} \\
R=\sqrt[3]{\frac{G M K}{\pi v^{2}}} \\
R=\frac{\left(6.67433 * 10^{-11}\right) *\left(8.99 * 10^{9}\right) *[(82 * 1.6726)+(126 * 1.6749)] * 10^{-27}}{3.1415 *(382,606)^{2}} \\
R=7.6874 * 10^{-13} \text { meters } \quad(75 \text {-sexies })
\end{gathered}
$$

This is the theoretical value of the lowest electron orbital radius of the lead atom.

Let's compare the theoretical radius obtained in (75-sexies) to the nuclear radius of lead atom given by the eq. (59), because in the reference [2] the comparison is done with regard to the nuclear radius $\left(R=1.2 * A^{\frac{1}{3}} \mathrm{fm}\right)$ :

$$
\frac{7.6874 * 10^{-13}}{7.1721 * 10^{-15}}=107.18
$$

So we have demonstrated that the formulae (75) and (75-quinquies) are correct, since the theoretical value of electron orbital radius given by the mentioned formulae is equal to about 100 times the nuclear radius of the lead atom (heavy atom).

It's worth to precise that the eq. (75-quinquies) expresses the lowest electron orbital radius because this is again the "ideal radius" of the electron orbit, where the orbital stability is maximum because at this distance from the nuclear center the gravitoelectric potential energy of the electrons equals twice their kinetic energy.

Since the calculations reported in the reference [2] tell us that the approximate ratio 100/1 between the lowest electron orbital radius and nuclear radius regards only the heavy nuclei, now we try to calculate the hydrogen electron orbit, i.e. the lightest nucleus, in order to demonstrate that the eq. (75) is again correct (indeed the resultant ratio we'll be greater than 100).

Let's use the velocity of the electrons of hydrogen atom obtained in the eq. (75-bis): $240,129 \mathrm{~m} / \mathrm{sec}$. 
Now let's calculate the lowest electronic orbital radius of the hydrogen atom using the formula (75-quinquies), where $v$ is equal to $240,129 \mathrm{~m} / \mathrm{sec}$ :

$$
\begin{gathered}
R=\sqrt[3]{\frac{K G M}{\pi v^{2}}} \\
R=\sqrt[3]{\frac{\left(8.99 * 10^{9}\right) *\left(6.67433 * 10^{-11}\right) *\left(1.6726 * 10^{-27}\right)}{3.1415 *(240,129)^{2}}} \\
R=1.7694 * 10^{-13} \text { meters }
\end{gathered}
$$

Let's compare the theoretical electronic orbital radius obtained above to the nuclear radius of hydrogen atom given by (59), and we'll see that the first is more than 100 times larger than the second (about 150 times):

$$
\frac{1.7694 * 10^{-13}}{1.21 * 10^{-15}}=146.23
$$

We have again demonstrated, therefore, that the formulae (75) and (75quinquies) are very likely correct, because they lead to the fact that the theoretical value of the lowest electron orbital radius is about 100 times larger than the nuclear radius only in heavy atoms, in agreement with the probabilistic predictions of quantum mechanics.

So now we can write better the formula (75-quinquies) where, we repeat, $v$ is equal to the right-hand side of (75), therefore let's replace in (75-quinquies) $v$ with the mentioned right-hand side:

$$
\begin{gathered}
R=\sqrt[3]{\frac{G K M}{\pi v^{2}}} \\
\rightarrow R=\sqrt[3]{\frac{G K M}{\pi *\left(c \sqrt{\frac{M \pi}{m K}}\right)^{2}}} \\
\rightarrow R=\sqrt[3]{\frac{G K M}{\left(\frac{\pi^{2} c^{2} M}{m K}\right)}} \\
\rightarrow R=\sqrt[3]{\frac{G K^{2} m}{\pi^{2} c^{2}}}
\end{gathered}
$$

As regards the dimensional analysis: 


$$
\begin{gathered}
R=\sqrt[3]{\frac{G K M}{\pi v^{2}}} \\
\mathrm{~m}=\sqrt[3]{\frac{\left(\frac{\mathrm{N} * \mathrm{~m}^{2}}{\mathrm{~kg}^{2}} * \frac{\mathrm{N} * \mathrm{~m}^{2}}{\mathrm{C}^{2}} * \mathrm{Kg}\right)}{\frac{\mathrm{m}^{2}}{\mathrm{~s}^{2}}}} \\
\mathrm{~m}=\sqrt[3]{\frac{\left(\frac{\mathrm{N}^{2}}{\mathrm{c}^{2}} * \frac{\mathrm{m}^{4}}{\mathrm{~kg}^{2}} * \mathrm{Kg}\right)}{\frac{\mathrm{m}^{2}}{\mathrm{~s}^{2}}}}
\end{gathered}
$$

Being $\frac{\mathrm{N}^{2}}{\mathrm{C}^{2}}=\mathrm{N}$, as demonstrated in reference [20], then:

$$
\begin{gathered}
\mathrm{m}=\sqrt[3]{\mathrm{N} * \frac{\mathrm{m}^{2} * \mathrm{~s}^{2}}{\mathrm{Kg}}} \\
\mathrm{m}=\sqrt[3]{\frac{\mathrm{Kg} * \mathrm{~m}}{\mathrm{~s}^{2}} * \frac{\mathrm{m}^{2} * \mathrm{~s}^{2}}{\mathrm{Kg}}} \\
\mathrm{m}=\sqrt[3]{\mathrm{m}^{3}} \\
\mathrm{~m}=\mathrm{m}
\end{gathered}
$$

Eq. (75-octies) expresses the lowest orbital radius of electrons: as we can see it doesn't depend on nuclear mass, but only on total mass of electrons $(m)$.

Anyway it's worth to specify that (75-octies) is not applicable to the galactic system, because here the potential energy of central system (proton star) is different from the potential energy concerning the space surrounding the central star, by the first being gravitational and the second being gravitoelectric (except the innermost part of the space surrounding the mentioned central star, where it is gravitational).

Let's verify, now, if our theory allows to determine also the maximum length of atomic radius, i.e. the highest electron orbit.

Let's take the hydrogen atom.

We have already seen that in order to obtain the maximum velocity of the electrons generated by the nucleus by means of the entanglement bond existing between nucleons and electrons, one has to impose in the formula of velocity (74) the condition that the radius be undimensional and equal to $R=1$.

Therefore, analogously, in order to determine the maximum extension of electron orbital radius at which it's possible to keep the orbital velocity of electrons 
constant, one has to impose in formula of the electron orbital radius (75-quinquies) the condition that the velocity equal to $v=1 \mathrm{~m} / \mathrm{s}$ [the dimension $\mathrm{m} / \mathrm{s}$ is necessary because in eq.( 75-quinquies) a dimensional constant lacks, differently from eq. (66) and (74), where there is the dimensional constant $c$ ], then eq. (75-quinquies) becomes:

$$
\begin{gathered}
R=\sqrt[3]{\frac{K G M}{\pi(1)^{2}}} \\
\rightarrow R=\sqrt[3]{\frac{K G M}{\pi}}
\end{gathered}
$$

In (75-novies) let's replace $M$ with the mass of hydrogen nucleus:

$$
\begin{gathered}
R=\sqrt[3]{\frac{\left(8.99 * 10^{9}\right) *\left(6.67433 * 10^{-11}\right) *\left(1.6726 * 10^{-27}\right)}{3.1415}} \\
R=6.836 * 10^{-10} \text { meters }
\end{gathered}
$$

This is the maximum distance at which the nuclear energy manages to move the electron at constant velocity, therefore it is the maximum extension of the atomic radius (as we'll explain in paragraph 5.13).

As we notice, the highest orbit of electrons predicted by (75-novies) is in good agreement with the sizes of the atom currently accepted, that are 5 order of magnitude larger than the nuclear radius (see pag. 11 of reference [4]).

In summary, the range of the electron orbital radius around the nucleus is from the minimum value given by (75-octies) to the maximum value given by (75-novies).

- 5.9 - Principle of specific asymmetry between gravitational potential energy and kinetic energy as an ideal condition of orbital stability

Here we will show that in our universe there exists a fundamental principle according to which the dynamic equilibrium between two (or more) bodies intended as a potentially perpetual orbit of one body around another one or around the mutual center of mass - will be the most stable, if the gravitational potential energy to which the orbiting body is subjected tends to be twice its kinetic energy, or twice the kinetic energy of the body which makes it move through an entanglement relationship. 
In other words, a body orbits in the most stable way (therefore potentially forever) around the attractor-repeller or the mutual center of mass if the following condition exists:

$$
-U=2 E_{k}
$$

where $E_{p}$ is the gravitational potential energy to which the orbiting body is subjected and $E_{k}$ is its kinetic energy or the kinetic energy of the entangled body responsible for its movement.

The balance between the two energies is "asymmetrical" because there must never be equality between them; is "specific" because a generic disproportion between the two energies is not sufficient, but a very precise level of inequality must be reached, i.e. the potential energy must be equal to twice the kinetic energy; moreover it is "ideal" because if the condition indicated in (76) is not reached, the orbit will not necessarily be unstable, but it will certainly be less stable.

This principle can be demonstrated by comparing the values of potential and kinetic energy both in the macroscopic world and in the microscopic one.

Let's start from the macrocosm, in particular from the solar system.

We know that in the solar system the gravitational potential energy is expressed by (52-bis), which we have seen to become, by virtue of the second principle of dynamics:

$$
U=-m * g * R
$$

Well, let's see if our assumption is true, i.e. if there exists a fundamental principle in our universe according to which, in order to reach the ideal condition of stability of the dynamic equilibrium between two (or more) bodies bonded by gravity force, gravitational potential energy to which a body is subjected must be equal to twice its kinetic energy.

If this is true, then in our solar system we will have that, for each planet, the following relation will be operating:

$$
-U=2 E_{k}
$$

where $E_{k}$ is the kinetic energy of the planet, that is equal to:

$$
E_{k}=\frac{1}{2} m v^{2}
$$

By replacing the left-hand side of (78) with the right-hand side of (77), and by also replacing $E_{k}$ with its value written in (79), we have that: 


$$
\begin{gathered}
m * g * R=2\left(\frac{1}{2} m v^{2}\right) \\
\rightarrow m * g * R=z\left(\frac{1}{z} m v^{2}\right) \\
\rightarrow g * R=v^{2} \\
\rightarrow \frac{G M}{R^{z}} * R=v^{2} \\
\rightarrow \frac{G M}{R}=v^{2} \\
v=\sqrt{\frac{G M}{R}}
\end{gathered}
$$

which is precisely the value of the orbital velocity of the planets, which demonstrates the existence of the principle in question at the macroscopic level.

This principle finds in turn a foundation in the principle of the least possible approach and the least possible distancing, which we have seen above be satisfied to a greater extent, the smaller the distance between the foci is, i.e. if eccentricity is as small as possible, and in fact the (79-bis) notoriously expresses the speed necessary to obtain an (almost) perfectly circular orbit.

It should be reiterated that, if the principle expressed in (76) is not respected, the orbit will not be unstable, but it will only be less stable, that is, it will have a higher eccentricity degree until it becomes hyperbolic if the potential energy equals the kinetic one.

Let's see if this principle also exists in the atom.

Let's start from the nucleus.

Let's assume that the nucleons (protons and neutrons) do not stand at rest inside the nucleus, but they move at the speed of light.

This is made possible in theory by the fact that, as we will see later, the sum of the volume of the nucleons is always lower than the volume of the nucleus itself (more or less in a ratio of $1: 3$ ), so it is reasonable to assume that this space serves to the nucleons to move.

Now, if the principle expressed by (76) also operates within the nucleus, then we'll have that the potential energy of the nucleons, expressed by the left-hand side of the (56-ter), and generated by attractive-repulsive field produced by the nucleons themselves, will be equal to twice the kinetic energy of the nucleons, so we can write: 


$$
\begin{aligned}
& \rightarrow \frac{4 k G M^{2}}{R^{3} \pi}=2\left(\frac{1}{2} M c^{2}\right) \\
& \rightarrow \frac{4 k G M^{2}}{R^{3} \pi}=M c^{2} \\
& \rightarrow \frac{4 k G M^{2}}{R^{3} \pi}=M c^{2} \\
& \rightarrow \frac{4 k G M}{R^{3} \pi}=c^{2} \\
& \rightarrow c=\sqrt{\frac{4 K G M}{R^{3} \pi}}
\end{aligned}
$$

And indeed, from the calculations, the right-hand side of the (80) is just equal to the speed of light, provided that one gives $R$ the value obtained by (57).

It is very probable, therefore, that the nucleons move within the nucleus at the speed of light.

But do the nucleons move with a straight or circular motion?

The presence of 4 before $g$ in the formula (55) of the strengthened potential energy possessed by the nucleus makes us incline for the second hypothesis, as this number can be justified only by hypothesizing the circular movement of the nucleons around the center of nucleus, since only from this type of motion the centripetal and centrifugal acceleration arise, to be added to the attractive gravitoelectric acceleration and to the repulsive gravitoelectric acceleration (in all they are 4 equal accelerations), as we have seen above.

But how is it possible that the nucleons move around the center of the nucleus if there is nothing in this point?

In other words, if the nucleons are in orbit around the center of nucleus, it means that there is nothing in the center, yet a body can orbit around another body or around the mutual center of mass, but it certainly cannot revolve around a point in which there is no other massive body.

How to solve the impasse?

The solution lies in (56-ter). Let's bring it back here:

$$
\frac{4 K G M^{2}}{R^{3} \pi}=M c^{2}
$$

The left-hand side of the above equation expresses, as mentioned several times, the gravitoelectric potential energy of nucleus. 
How is it possible that it depends on the square of the mass of the nucleons?

Usually the potential energy $(U)$ to which a body revolving around an attractor body is subjected depends not only on the mass of the attractor, but also on the one of the attracted body:

$$
\begin{gathered}
U=-m * g * R \\
\rightarrow U=-m * \frac{G M}{R^{2}} * R
\end{gathered}
$$

where $m$ is, in fact, the mass of the orbiting body and $M$ the mass of the central body.

How is it possible, then, that the potential energy of the nucleons - the central body — depends only on their mass (squared), and not also on the mass of the orbiting body?

The only possible explanation, in my opinion, is to believe that the nucleon behaves simultaneously both as orbited body and as orbiting body, namely that stays at same time both at the center of the nucleus and also in orbit around it at the distance given by (57).

In other words, it is as if the nucleon, placed at the center of the nucleus, projected its own shadow onto the "shell" of the nucleus, and this shadow moved at the speed of light around the central nucleon (or nucleons).

The fact that the orbiting particle is similar to a shadow of the central nucleon also allows to explain how it is possible that the central nucleon remains motionless, whereas its alter-ego moves at the speed of light around it, whereas it would be difficult to solve with recourse to pure wave-particle duality, which would not explain the immobility of the central nucleon, on the one hand, and the mobility of its orbiting alter-ego, on the other; moreover, the recourse to the idea of "orbiting shadow nucleon", to be understood as an uncharged alter-ego of the central one, also allows to explain why, as results from the experiments, the density of the nucleus is concentrated in its center.

In this perspective the so-called Copenhagen interpretation of the doubleslit experiment would seem to be confirmed (albeit with the clarification we will give below), according to which the same photon, thrown on a very thin gold plate, crosses at the same time two slits made on such gold plate, thanks to the dual nature of the photon, as opposed to the interpretation of De Broglie, who instead 
proposed that the photon has no dual nature (both of wave and of particle), but that the wave is something different from the particle, so the experiment of double slit, in his opinion, should be interpreted as meaning that a slit is crossed by the photon and the other is crossed by the wave, on which the photon would move.

In other words it is probable that the two phenomena are both attributable to the particular "dual" nature of the fundamental particles, to which at this point the nucleons (protons and neutrons) must also belong, so that the nucleon must be considered a particle and a wave at the same time, but with the particularity of moving not in unison with the wave, but projecting its shadow through the wave, and consequently find itself in two different places at the same time (both in the center of the nucleus and in orbit onto its shell), even if I realize that any attempt to describe this very strange phenomenon of wave-particle dualism can appear clumsy.

This would confirm the theory of the "dual" nature of the fundamental particles proposed by quantum mechanics.

After all, the experiments (see reference [1] and [15]) show that:

$>$ the nucleons doesn't appear as point-like particles, in the sense that they show no well-defined surface, according to their dual nature emerged in the present work;

$>$ they have a high angular momentum, as evidence of their circular movement and of their high velocity, in agreement with the results reached here.

But are the real nucleons, located at the center of the nucleus, aggregated or disjointed?

I'll give a more precise answer in the paragraph 5.15, n. 2).

The full nuclear matter radius given by (57) can be defined as the "ideal" radius of the nucleus - equal to twice the nuclear radius expressed by (59) - by being this the dimension of the radius at which the nucleus achieves maximum stability, because only with this value of the radius the strengthened potential energy expressed by the left-hand side of the (56-ter) perfectly equals twice the kinetic energy of the nucleons $\left(E=M c^{2}\right)$, fully satisfying the principle of specific asymmetry between the two energies stated in the eq. (76). 
The full nuclear matter radius cannot be equal to the radius resulting from the scattering of the incident beam of particles, i.e. that given by the eq. (59), because otherwise the potential energy would exceed twice the kinetic energy of the nucleons, with the risk of implosion of the nucleus itself.

I cannot determine what is the limit beyond which the overcoming of the potential energy with respect to double the kinetic one involves the implosion of the nucleus, although it is reasonable to hypothesize that this limit coincides with the distance at which the shadow nucleon (or nucleons) is no longer able to move.

However, it is possible to predict with greater theoretical certainty the limit beyond which the explosion of the nucleus should occur, that would be, using a logic process similar to the one used to determine the escape velocity of the planets, when the potential energy of the nucleus is reduced until to equal the kinetic energy of the nucleons of which it is composed:

$$
\begin{gathered}
-U=E_{k} \\
\frac{4 G K M^{2}}{R^{3} \pi}=\frac{1}{2} M c^{2} \\
\frac{8 G K M^{2}}{R^{3} \pi}=M c^{2} \\
R^{3} \pi c^{2}=8 G K M \\
R=\sqrt[3]{\frac{8 G K M}{\pi c^{2}}}
\end{gathered}
$$

The value expressed by the eq. (81), greater than the one given by the eq. (57) is, therefore, the length of the nuclear radius at which the gravitoelectric potential energy of the nucleus equals the kinetic energy of the nucleons, implying the loss of identity of the nucleus as self-interacting system, and probably protons and neutron decay in photons with an associated huge amount of energy (proton decay + beta decay).

As already mentioned, the reasoning just made is analogous to what is normally done to theoretically determine the escape velocity of planets, where, for this purpose, the equality between the gravitational potential energy of the planet and its kinetic energy is imposed:

$$
F_{g} * R=\frac{1}{2} m v^{2}
$$




$$
\begin{gathered}
m * g * R=\frac{1}{2} m v^{2} \\
m * \frac{G M}{R^{2}} * R=\frac{1}{2} m v^{2} \\
m * \frac{G M}{R^{z}} * R=\frac{1}{2} m v^{2} \\
\frac{2 G M}{R}=v^{2} \\
V=\sqrt{\frac{2 G M}{R}}
\end{gathered}
$$

We now use the same reasoning to determine the consequences of equality between the potential revolution energy of electrons and the kinetic energy.

According to the principle of specific asymmetry hypothesized here, the potential energy to which the electrons are subjected must be equal to twice the kinetic energy of the entangled body responsible for their movement: in this case, indeed, the second part of the aforementioned principle is applicable (... "or twice the kinetic energy of the body that makes it move through an entanglement relationship"), by we having hypothesized that the electron movement is connected to the movement of the nucleons, in the sense that their potential energy has to be twice the kinetic energy of nucleons in order to obtain a stable orbit, and that this connection occurs without energy dispersion and without any time of information transmission.

Consequently we must equal the revolution potential energy of electrons, expressed by the left-hand side of the eq. (73), to the kinetic energy $E_{k}$ of the nucleons:

$$
v^{2} * \frac{m K}{R^{2} \pi}=\frac{1}{2} M c^{2}
$$

where $M$ is the mass of nucleons and $m$ the mass of electrons;

$$
\begin{gathered}
\rightarrow v^{2}=\frac{M c^{2} R^{2} \pi}{2 m K} \\
\rightarrow v=c R \sqrt{\frac{M \pi}{2 m K}}
\end{gathered}
$$

By imposing again $\mathrm{R}=1$ for the reasons indicated in the paragraph 5.8, we'll have:

$$
v=c \sqrt{\frac{M \pi}{2 m K}}
$$




$$
v=169.797 \mathrm{Km} / \mathrm{sec}
$$

This is the theoretical speed at which the electron of the hydrogen atom should be forced to move in order to emit electromagnetic radiation in the form of a photon emission, without changing its distance from nucleus.

It's important to state that the velocity given by the eq. (82) is not the escape velocity of electrons, but the velocity at which the electron emits electromagnetic radiation.

In particular, note that the speed given by the eq. (82) is lower than the one imposed by the attractive-repulsive field generated by the nucleons (which in 75-bis we have seen to be, for the aforementioned hydrogen atom, about 240 $\mathrm{km} / \mathrm{sec}$ ), so that the surplus of energy that would be created instantaneously due to the entanglement bond presumably existing between nucleons and electrons, would cause the emission, by the electron whose speed is forcibly reduced, of energy in the form of electromagnetic radiation (but maybe it would be more appropriate to define them gravitoelectric radiation).

But the forced reduction of the velocity of the electron is only a theoretical issue.

In the facts, it happens that the electron whose energy is increased, by the absorption of a photon, despite keeping constant its velocity of revolution around the nucleus, moves onto a lower energy level, i.e. going onto an orbit farther from the nucleus.

In fact later we will see that, assuming the analogy between the atom and the galaxy - at least under the profile of the gravitoelectric revolution field that would exist in the space surrounding the central nucleus - it is not hazardous to assume that the speed of the electrons, like the one of stars, remains constant, despite moving onto a higher orbit, farther from the nucleus, and we will see that this is abstractly achievable in a model based on the pendulum principle, if we think about the possibility that the repulsive gravitoelectric force, even if decreases in intensity proportionally to the attractive gravitoelectric force, formed with the latter an angle always greater in relation to the increase of distance from the nucleus, so as to keep constant the $F_{r x}$ force, and therefore constant the orbital velocity. 
In this case, the emission of the photon would be due to the fact that the electron has moved to an outermost orbit, where, despite a lower potential energy, there is an equal revolution speed (like we shall see later in the paragraph 5.13) and by means of this emission the electron loses energy and comes back in the stability zone (see the next two pages).

In particular the distance from nucleus of hydrogen atom at which the electron would emit electromagnetic (or, maybe, more likely, gravitoelectric) radiations would be given by the formula (75-quinquies), with $v$ equal to the value expressed by the eq. (82):

$$
\begin{gathered}
R=\sqrt[3]{\frac{G K M}{\pi v^{2}}} \\
R=\sqrt[3]{\frac{\left(6.67433 * 10^{-11}\right) *\left(8.99 * 10^{9}\right) *\left(1 * 1.6726 * 10^{-27}\right)}{3.1415 *(169,797)^{2}}} \\
R=2.2293 * 10^{-13} \text { meters }
\end{gathered}
$$

It's essential to specify that this is not the distance at which the electron should move if its speed was 169,797 meters/sec, but the distance at which the electron would move at 169,797 meters /sec if the gravitoelectric repulsive force formed with the gravitoelectric attractive force the same angle as that formed between them at the distance obtained in the eq. (75-septies): the reason of this will be explained in paragraph 5.13 .

The same result is also obtained by imposing the equality between the potential energy of electrons and their kinetic energy, taking as $v$ the value given by (75-bis), i.e. $240,129 \mathrm{~m} / \mathrm{sec}$.

$$
\begin{gathered}
\frac{G K M m}{R^{3} \pi}=\frac{1}{2} m v^{2} \\
R=\sqrt[3]{\frac{2 G K M}{\pi v^{2}}} \\
R=2.2293 * 10^{-13} \text { meters }
\end{gathered}
$$

So, in the eq. (82-bis), we can replace $v$ with the right-hand side of the eq. (75):

$$
R=\sqrt[3]{\frac{2 G K M}{\pi *\left(c * \sqrt{\frac{M \pi}{m K}}\right)^{2}}}
$$




$$
\begin{gathered}
R=\sqrt[3]{\frac{2 G K^{2} m}{\pi^{2} * c^{2}}} \\
R=\sqrt[3]{\frac{2 *\left(6.67433 * 10^{-11}\right) *\left(8.99 * 10^{9}\right)^{2} *\left(1 * 9,11 * 10^{-31}\right)}{(3.1415)^{2} *(299,792,458)^{2}}} \\
R=2.2293 * 10^{-13} \text { meters }
\end{gathered}
$$

The equation (82-ter) expresses therefore the orbital radius of electron from which it begins to emit gravitoelectric radiation in the form of photon release.

But at this point a question arises: if the velocity of electrons is constant, how it's possible that the system nucleus-electron is stable?

In other words, the orbit is stable if the potential energy is equal to twice the kinetic energy of orbiting body, but, if the velocity of electrons doesn't decrease with the distance, at a certain point (i.e. the radius expressed by the eq. 82-ter) the kinetic energy of electrons will be equal to the potential energy, and the dynamic equilibrium between them should break.

Why does this not happen in the atom?

The electron can orbit, in fact, until the distance given by the equation (75novies), larger than that predicted by the equation (82-ter).

How can this happen?

The only possible explanation to this question is to believe that the entanglement bond existing between nucleons and electrons guarantees the atom's stability. In fact, by we having imposed in the eq. (71) the equality between potential energy of the electrons and twice the kinetic energy of nucleons, we have derived the velocity of all the electrons, given by the ratio between the total mass of the nucleons and the total mass of the electrons, so when this velocity is respected and the electron moves within the maximum radius given by the eq. (75novies), the system nucleus-electron is still balanced, even though is less stable.

This entanglement bond also manifests itself with the emission of photons when the distance given by the eq. (82-ter) is exceeded: in this way the electron loses energy and returns to the innermost level, the most stable one, because here the potential energy of the electrons is greater than their kinetic energy.

In other words, when the electron is in the instability zone, i.e. beyond the distance given by the eq. (82-ter), the nucleus "tells" the electron to release a 
photon in order to lose energy and to come back to the stability zone (the lowest one), and a possible indirect evidence of this instantaneous communication is precisely the fact that the (theoretical) photon mass is so similar to the proton mass squared.

In fact the theoretical mass [5] [6] of the photon is about $2 * 10^{-54} \mathrm{~kg}$, and this value is very near to the proton mass squared, i.e. $2.7976 * 10^{-54} \mathrm{~kg}$, so it's reasonable to hypothesize that the photon is emitted by the electron through the entanglement bond existing between the latter and the nucleus, in which we have seen that the strengthened potential energy depends precisely on the mass of the nucleons squared.

Consequently, we can hypothesize that, when the nuclear radius or the neutron star radius reach the extent given by the eq. (81), it doesn't occur an explosion, but most likely an emission of light with an associated huge release of energy.

In this case, in fact, the break of dynamic equilibrium between the central real particles and the shadow particles is physiological, because it is due to the achievement of the equality between potential energy and the kinetic one, even if it's not easy to say what are the causes which determine this equality.

The same applies to the proton star (black hole), in which the equality between the strengthened potential energy — given, as we will see in the paragraph 5.11 , by the left-hand side of the eq. (83) - and kinetic energy of orbiting real protons implies:

$$
\begin{gathered}
\frac{4 G M^{2}}{R}=\frac{1}{2} M c^{2} \\
R=\frac{8 G M}{c^{2}}
\end{gathered}
$$

This is the radius of the proton stars at which it occurs an emission of light.

We have therefore demonstrated, or proved to demonstrate, the existence of the universal principle of specific asymmetry between gravitational potential energy and kinetic one as an ideal condition of stability of the orbit of a body around the attractor-repeller or around the mutual center of mass, which, on the one hand, makes it possible to understand what the nuclear energy formula $E=M c^{2}$ derives from, but, on the other, it highlights all the limits of the principle of energy 
conservation, because one question remain open to which it is difficult to give an answer:

1) By we having demonstrate that in the nucleus the potential energy constrains nucleons to move around, as a centripetal force, therefore not as a revolution energy, a question arises: what determines the movement of the nucleons within the nucleus at the speed of light?

\section{- 5.10 - The radius of neutron stars: differences with respect to the proton stars}

Neutron stars (to which the pulsars also belong) are, as the name suggests, celestial bodies made up solely of neutrons.

The characteristic of neutron stars is to have a very high density: in fact a typical neutron star has a mass of about 1.4 solar masses, but has a radius of just $10-15 \mathrm{~km}$, very small if compared to the radius of our Sun, equal to about $696,000 \mathrm{~km}$.

Such a high density can only be found inside the atom's nucleus, so neutron stars are an important test to verify the validity of the theory here exposed.

Let's try then to calculate, by means of (59), the radius of a typical neutron star, i.e. with mass equal to about 1.4 solar masses.

For convenience, we report below (59):

$$
R=\frac{1}{2} * \sqrt[3]{\frac{4 K G M}{\pi c^{2}}}
$$

where $M$ is the mass of the neutron star, equal, as we said, to $1.4 * 1.9891 *$ $10^{30} \mathrm{~kg}$.

$$
\begin{gathered}
\rightarrow \quad R=\frac{1}{2} * \sqrt[3]{\frac{4 *\left(8.99 * 10^{9}\right) *\left(6.67433 * 10^{-11}\right) *\left(1.4 * 1.9891 * 10^{30}\right)}{3.1415 * 89,875,517,873,681,764}} \\
R=14,356 \text { meters } \\
R=14.3 \mathrm{Km}
\end{gathered}
$$

As we note, (59) is perfectly applicable also to neutron stars, by being in full agreement with astronomical estimates, thus confirming not only the universal character of the theory here exposed, but also the actual similarity between neutron star and the nucleus of the atom, already detected by astronomers. 
In truth, the one resulting from the eq. (57) should be the full matter radius of the neutron star (see paragraph 5.5), including the shadow orbiting neutrons, whereas the one given by the eq. (59) should be the extension of the central core radius, made up of the real neutrons.

Assuming the similarity with the nucleus of the atom, it is possible to hypothesize for neutron stars, like we have done for the atomic nucleus, that neutrons move within the star at the speed of light.

In particular, the central neutrons, aggregated thanks to the gravitational (or more likely gravitoelectric) force, would have a "dual" nature, both corpuscular and undulatory, which would replicate the same conditions seen for the nucleons, so that their "shadows" (i.e. their mobile alter-egos) would revolve around the central neutrons at the speed of light at the distance given by (57).

We can therefore hypothesize that, also with respect to neutron stars, one can applies the principle, stated by (76), of specific asymmetry between the potential energy of the neutrons and their kinetic energy, so the radius given by (57) would be the "ideal" radius of the neutrons star, namely the radius that guarantees maximum stability of the star, since it makes the strengthened potential energy of shadow neutrons equal to twice their kinetic energy.

Accordingly, if the radius increases with respect to the value given by (57), the star would become less stable, until it explodes once the potential energy of the neutrons is reduced until to equal their kinetic energy, i.e. when the radius assumes the value expressed by (81).

It's very likely, therefore, that neutrons of pulsar generate a strengthened gravitoelectric attractive-repulsive field capable of constraining the "shadow neutrons" to revolve in perpetuity around the center of the star occupied by the "real" central neutrons.

The gravitoelectric nature of the strengthened attractive-repulsive field generated by the central neutrons of pulsar shows us that these differ from the proton stars (the so-called black holes), since the protons remain confined within the "black hole" radius because of the strengthened attractive-repulsive field having a gravitational nature (not gravitoelectric), generated by protons themselves.

Another difference could be the fact that, in the neutron star, the shadow 
particles orbit around their corresponding "real" alter ego, instead, in the proton stars, the real particles orbit around the shadow particles, which this time are located in the center of the star.

This can explain the reason why the black hole is stable, presumably because among central shadow particles there is no repulsive force, but only attractive one.

This central lump of shadow particles would generate the attractive-repulsive gravitational strengthened field that would be able to contain the "real" orbiting protons within the radius expressed by (50).

These real orbiting protons, moving at speed of light around their central shadow particles, would have - unlike the shadow particles orbiting around the center of atomic nucleus as well as around the center of neutron star - all the property of the protons, i.e. a mass and a charge, so it's reasonable to argue that they will produce kinetic energy of mass $E=\frac{1}{2} M c^{2}$, and kinetic energy of charge $E=\frac{1}{2} Q c^{2}$.

The second kind of energy would be a new kind of energy, never tested before, so this statement can only have a speculative character, even though, by comparing the gravitoelectric revolution potential energy of stars to twice such "charge kinetic energy" of protons orbiting inside the black hole, like we have done in (64), we are able to explain the orbital velocity of the stars around the central black hole of the galaxy, as we have seen in (70-bis).

So we can conclude that, if there exists the "kinetic energy of charge" possessed by the real protons orbiting at speed of light around their corresponding shadow particles lump located at the center of the black hole, then we can suppose that this energy is linked, through an entanglement bond, to the gravitoelectric attractiverepulsive field of revolution, which makes the stars to move around the black hole to a constant velocity.

Another difference between the two stars seems to be the fact that the neutron star causes the rotation of the planets around it by virtue of an attractive-repulsive field of gravitational nature, therefore with decreasing speed in relation to the increase of distance from the central star, instead the proton star determines the rotation of the stars around it by virtue of an attractive-repulsive field of gravitational nature only in 
the proximity zone, which then turns, with regards to the stars located at a certain distance from the center, into a gravitoelectric field of revolution (resulting in almost constant velocity of galaxy stars).

A possible element in common between the two stars could be the fact that the

"kinetic energy of mass" $E=\frac{1}{2} M c^{2}$ possessed both by the orbiting real protons due to the fact that they also have a mass, not only a charge - and by the orbiting shadow neutrons should theoretically be related, through an entanglement relationship, to the gravitoelectric attractive-repulsive field of revolution that makes the microscopic particles orbit in the innermost part of space surrounding the black hole and surrounding the neutron star (presumably the electrons, like it happens in the atom, in which the electrons are moved by virtue of the entanglement bond existing with the kinetic energy of mass of the orbiting shadow nucleons).

Therefore, both stars have some aspect in common with the atom as a whole, but they are not perfectly superimposable to it: the neutron stars is similar to the nucleus of the atom only in terms of density; the proton stars, on the contrary, can be assimilated to atoms only with regard to the nature (gravitoelectric) of the forces-field generated in its surrounding space, but not also with respect to the density, which remains much lower than the one of the atomic nucleus, by being the real protons confined within the black holes by the strengthened attractive-repulsive field having a gravitational nature and generated by the central shadow protons, less strong than the strengthened gravitoelectric attractive-repulsive field existing in the nucleus of atom generated by the central real nucleons that makes the shadow orbiting nucleons be confined within the nucleus itself.

\section{- 5.11 - The characteristics of shadow particles connected to the nucleons}

If we agree with all the above, it is possible to reconstruct the characteristics of the shadow particles related to protons and to neutrons:

1) The mass of the shadow particle is equal to the mass of its corresponding real particle;

2) despite this, the shadow particle is not able to change the center of mass around which there subsists the orbiting movement, which remains located in the center of nucleus, not at the intermediate distance between the 
shadow particle and the real one, precisely because it's a case of the selforbiting phenomenon;

3) they can assume the role of orbiting particles (as it occurs in the nucleus of the atom and in the neutron stars) or of orbited particles (as it happens in the black holes);

4) in both cases they are not suitable for reflecting the light that hits them;

5) they are indirectly detectable in the scattering experiments of alpha-particles (positively charged). These experiments, indeed, show that the alfaparticles beam is only partially diffracted by the target nucleus, in the sense that a part of this particles is absorbed in the collision, "disappearing" (the word is used in reference [2], pag. 514). This is weird because the incident particles and the target nucleus are both positively charged, so one would expect that the incident particles to be totally diffracted by the target nucleus, without any absorption. For these reason the nuclear "optical model", in order to solve the problem from the mathematical point of view, assumes that the nuclear potential to be in part real and in part "imaginary", and this second part of the potential would be responsible for the partial absorption of particles beam.

Well, we can reasonably suppose that the imaginary part of optical potential, which allows to better fit the data, can find a physical explanation in the shadow particles proposed in the present work, by we having already arrived at the conclusion that this particles are not charged and are unsuitable for reflecting or diffracting the photons and, at this point, each kind of beam of particles.

After all, it has been shown [3] that the imaginary part of optical potential is proportional to the real part, which is in accordance with our theoretical results, since our nuclear radius given by (57) being exactly equal to twice the nuclear radius deduced from the scattering experiments (hence the proportionality ratio is $1: 1)$. In the latter reference in fact it's reported a maximum proportionality factor of 1 (see fig. 5, pag. 18).

Furthermore, in the reference [13] it's shown that, in the heavy atoms, the alpha-particles absorption becomes important at a distance equal to twice 
the nuclear radius, in agreement with our theory.

In the reference [14], then, it has been pointed out that, in order to calculate the total cross section, when an alpha-particles beam hits a target nucleus, it's necessary to take into account not only the scattering cross section but also the reaction cross section, and the final result is that, when the absorption is maximum $\left(\eta_{l}=0\right)$, the scattering cross section and the reaction cross section are identical, hence the total cross section turns out to be twice the geometric (i.e. the scattering) cross section. In this reference it's reported in particular that "In this situation we can say that the part of the beam which is diffracted has to be the same as the part that is absorbed, justifying the equality of the two cross sections".

At the same result other researches [17] [18] arrive in the case of a beam of antiprotons (negatively charged) fired on a target nucleus, in which case it has been observed that the absorption of the incident antiprotons beam is significant even at distances as large as twice the nuclear radius, as further confirmation of the theory proposed in the present work.

6) when they assume the role of orbiting particles (i.e. in the atomic nucleus and in neutron star), they move at the speed of light and produce only mass kinetic energy $E=\frac{1}{2} M c^{2}$ (not also charge kinetic energy $E=\frac{1}{2} Q c^{2}$ ), which is connected, through an entanglement bond, to the electrons placed around the nucleus and around the neutron star, in the sense that the electronic orbits will be as stable as possible if their total gravitoelectric revolution energy (without 4 and depending on $M * m$ ) will be twice the total kinetic energy of the shadow particles which are orbiting within the central system (atomic nucleus, or neutron star);

7) when they play the role of orbited central particles (i.e. in the proton stars), they produce - consistently with the absence of charge resulting from the previous point — an attractive-repulsive strengthened field having an exclusively gravitational nature, that binds the corresponding real particles, which are moving at the speed of light by their nature, to revolve around the central shadow particles. In fact, if we place the equality between the strengthened gravitational potential energy seen in the (45-bis) and twice 
the kinetic energy of mass of protons, we will have exactly the radius of the black hole that we have obtained in (50):

$$
m * 4 g * R=M c^{2}
$$

now we replace $m$ with $M$ because we have assumed that the protons of black hole are self-orbiting particles:

$$
\begin{gathered}
\rightarrow M * 4 g * R=M c^{2} \\
\rightarrow \frac{M 4 G M}{R^{2}} * R=M c^{2} \\
\rightarrow \frac{4 G M^{2}}{R}=M c^{2} \\
\rightarrow \frac{4 G M}{R}=c^{2} \\
\rightarrow 4 G M=R c^{2} \\
\rightarrow R=\frac{4 G M}{c^{2}}
\end{gathered}
$$

8) in this latter case, once the maximum distance at which the real particles can orbit is exceeded (i.e. beyond the radius of the black hole), the central shadow particles generate a gravitational attractive-repulsive field having a non-strengthened (namely a non-selfinteracting) nature, which implies due to the differences in pressure deriving from the clash between attractive gravity and repulsive gravity in relation to the distance from the center - the revolution of the celestial bodies inserted therein at decreasing speed in relation to the increase of distance from the center, which then, reached a certain distance from the aforesaid center, turns into a gravitoelectric attractive-repulsive field of revolution, due to the "kinetic energy of charge" $\left(E=\frac{1}{2} Q c^{2}\right)$ of the real protons orbiting within the proton star around their corresponding central shadow particles, energy which is connected, though an entanglement bond, to the stars of the galaxy, in the sense that the stellar orbits will be as stable as possible if their total gravitoelectric revolution energy will be equal to twice the charge kinetic energy of the real protons orbiting within the proton star.

At this point a question arises: in the previous point 7) we have seen that the gravitational potential energy operating in the black hole is the same as that operating in the phenomenon of perihelion precession, but how is this possible? 
In other words, in the perihelion precession we have seen that the cause of the non-arising of the $F_{r x}$ force is the principle of conservation of energy, which prevents the planet, once arrived in the perihelion, from approaching further the sun, forcing it to move away from the star as little as possible, which is achieved precisely by the alignment between the two opposed forces (the attractive and the repulsive one), and then, ultimately, by the failed-birth of the $F_{r x}$ force.

But all this cannot happen also in the black hole, because here there exists a strengthened energy during the whole path of the real orbiting protons, whose speed is not caused by the attractive-repulsive field, but it is only constrained by this field, so there is no reason for the principle of conservation of energy to come to the aid, which is a limitation regarding the principle of the minor possible approach to the attractorrepeller, but the latter principle applies only in presence of revolution energy (or of revolution movement due to different levels of pressure, like it happens in gravitational attractive-repulsive field), which instead does not subsist, it is repeated, inside the black hole, therefore it is asked what is the cause that prevents the arising of the force $F_{r x}$ in the black hole.

The only possible answer is to believe that the principle that prevents the birth of the aforementioned force is the impossibility of overcoming the speed of light, which would instead be overcome if the protons, already endowed by their own nature with the same speed of photons (about 300 thousand $\mathrm{km} / \mathrm{sec}$ ), received, by virtue of the birth of the $F_{r x}$ force, a further acceleration.

A similar reasoning applies to the gravitoelectric strengthened field that there exists in the nucleus of the atom and in the neutron star, where the two gravitoelectric forces (attractive and repulsive), even if they arise misaligned, become aligned as soon as the orbiting particle, which travels at the speed of light by its own nature, occupies a determined point of the orbit, otherwise it would receive a further acceleration due to the $F_{r x}$ force: in this perspective it's more appropriate to say that, in this case, it occurs a suppression rather than a failed birth of $F_{r x}$ force.

\section{- $\quad 5.12$ - The self-orbiting phenomenon: mathematical tests}

It is now necessary to demonstrate mathematically the self-orbiting phenomenon which above we have hypothesized to exist within the nucleus of atom, 
inside the black hole and inside the neutron stars.

Let's start from the nucleus of atom, and precisely from the equation (56-ter):

$$
\frac{4 K G M^{2}}{R^{3} \pi}=M c^{2}
$$

Let's check, by means of calculations, if the above equation is true.

Let's consider the hydrogen atom, whose nucleus contains only one proton and let's replace in the aforementioned equation the values of $K, G, M, R$ and $c$, where $R$ is the ideal radius of nucleus, namely the one expressed by (57), and $M$ is the mass of proton.

$$
\begin{gathered}
\frac{4 *\left(8.99 * 10^{9}\right) *\left(6.67433 * 10^{-11}\right) *\left(1.6726 * 10^{-27}\right)^{2}}{\left(2.4167 * 10^{-15}\right)^{3} * 3.1415}=\left(1.6726 * 10^{-27}\right) *(299,792,458)^{2} \\
\rightarrow 1.4924 * 10^{-10}=1.4924 * 10^{-10}
\end{gathered}
$$

Now let's consider the black hole, and check whether the equation (83) is true:

$$
\frac{4 G M^{2}}{R}=M c^{2}
$$

Let's replace in the above equation the values of $G, M, R$ and $c$, where $R$ is black hole radius expressed by (50) and $M$ is the mass of black hole of Milky Way, equal to $3.75 * 10^{6}$ solar masses.

$$
\begin{gathered}
\frac{4 *\left(6.67433 * 10^{-11}\right) *\left(3.75 * 10^{6} * 1.9891 * 10^{30}\right)^{2}}{22,157,162,000}=\left(3.75 * 10^{6} * 1.9891 * 10^{30}\right) *(299,792,458)^{2} \\
\rightarrow 6.7039 * 10^{53}=6.7039 * 10^{53}
\end{gathered}
$$

Finally, let's have a look at the neutron stars, in particular by invoking again the equation (56-ter), which above we have seen to be applicable even to the type of stars in question:

$$
\frac{4 K G M^{2}}{R^{3} \pi}=M c^{2}
$$

Let's replace in the above equation the values of $K, G, M, R$ and $c$, where $R$ is the ideal radius of neutron star given by (57) and $M$ is the mass of a typical neutron star, equal to 1.4 solar masses.

$$
\begin{gathered}
\frac{4 *\left(8.99 * 10^{9}\right) *\left(6.67433 * 10^{-11}\right) *\left(1.4 * 1.9891 * 10^{30}\right)^{2}}{(28,712)^{3} * 3.1415}=\left(1.4 * 1.9891 * 10^{30}\right) *(299,792,458)^{2} \\
\rightarrow 2.5027 * 10^{47}=2.5027 * 10^{47}
\end{gathered}
$$


We have thus demonstrated - based on the fact that the strengthened potential energy (left-hand side of formula 56-ter and of formula 83) depends on the square of the mass of the central particles, and on the fact that this energy is equal to twice the kinetic energy of mass of orbiting particles, thus satisfying the principle of specific asymmetry enshrined in (76) - that in the atomic nucleus, in the black hole (proton star) as well as in the neutron star there exists the self-orbiting strange phenomenon, according to which one or more elementary particles, in particular protons and neutrons, revolve around themselves, with the difference that in the atomic nucleus and in neutron stars the shadow particles revolve around the real ones located in the center, whereas in the proton star the real particles very likely revolve around the central lump made up of shadow ones.

In either case, the orbiting particles move at the speed of light.

After all, as regards the black holes, the most recent researches [8] show that the existence of orbiting particles inside the black hole and around it (in particular photons) is compatible not with a singularity, but with a De Sitter center, in particular with a model in which the black hole is composed of a lump of solitons, namely a lump of self-interacting particle (so called G-Lump).

\section{- 5.13 - The reason of non-decreasing velocity of stars and of electrons}

It's time to solve another enigma: how is it possible that the velocity of the stars and electrons (assuming the atom-galaxy similarity) remains constant, even if the distance increases, despite the revolution energy decreases depending on the increase of distance?

In other words, if the gravitoelectric revolution energy to which the stars of the galaxies and the electrons of the atoms are subjected decreases with the increase of distance from the central nucleus, how is it possible that the orbital velocity remains constant?

The pendulum model proposed here allows once again to give an answer, if we think of the possibility that the $\overrightarrow{F_{r}}$ force had, with the increase of distance, an orientation more and more tilted with respect to the $\overrightarrow{F_{a}}$ force, i.e. if the angle between the repulsive and the attractive force increased with distance to an extent sufficient to keep the $\overrightarrow{F_{r x}}$ force, and therefore the orbital velocity, constant. 
In this way, although the energy of revolution decreases, the revolution speed remains constant, and this is not a paradox, because if hypothetically one succeeds in making the repulsive force to assume, in all points of the attractive-repulsive field, always the same angle with respect to the attractive force, regardless of the distance from the nucleus, the inner electrons would orbit at higher speed than the outer ones.

What counts, in order of quantifying the energy of revolution, is the ontological potentiality of a given point in the field to make the electron move at a certain speed, no matter if, due to a mere factual inconvenience (the increasing angulation of the repulsive force with respect to the attractive one dependently on the increase of distance from the nucleus), the revolution velocity of the electrons remains constant: a certain point of the attractive-repulsive field will still have the abstract and ontological potentiality to move the electron at an orbital speed higher than the speed at which another point, farther from the nucleus, would move it.

However, the angulation of the repulsive force with respect to the attractive one cannot grow indefinitely, but will find a limit in the principle of the least possible approach and the least possible distancing, which precludes the orbiting body from moving to the adjacent point characterized by an equal energy value — that is, to the point equidistant from the center, thus describing an arc of circumference imposing it to move to the adjacent point characterized by the least strong, or least weak possible energy value, describing an elliptical orbit.

In other words, the atom and the galaxy will extend up to that point beyond which the repulsive gravity force, in order to keep constant the orbital velocity (respectively of electrons and of stars), should have an orientation orthogonal to the orientation of attractive gravity force, which, as we said, is precluded by the necessary ellipticity of the orbit.

This is the reason why, even if the gravitoelectric potential energy operates up to an infinite distance, the atom and the galaxy do not have an infinite extension.

\section{- $\quad 5.14-\underline{\text { The velocity of nucleons }}$}

At this point a question arises: are we sure that the nucleons move within the nucleus at the speed of light?

The momentum of nucleons usually detected in the experiment is about 250 
Mev/c (the so colled Fermi momentum) which is not sufficient to assert that they are moving at the speed of light, but a recent research [19] has revealed that in some collision the missing momentum of the knockout nucleon can be up to $1,000 \mathrm{Mev} / \mathrm{c}$, which can allow one to infer a very high velocity of nucleons, for sure approaching the speed of light, and it is not excluded that further studies will give more clarifications and confirmations about that.

- 5.15 - The gravitoelectrostatic force: differences with the electrostatic

\section{force and with the gravitational force}

If one agrees with all the above, the gravitoelectrostatic force should be characterized by the following features:

1) It acts among fundamental particles (assuming that fundamental particles are those which constitute the fundamental system of the universe, namely the atom, therefore in this perspective also protons and neutron can be considered fundamental particles). It doesn't act between non-fundamental particles, among which the electrostatic force acts;

2) It acts among the nucleons in the nucleus, in which case it is exclusively attractive (under the assumption that the neutrons neutralize the repulsive force between protons), and between the nucleons, on the one hand, and the electrons, on the other, by means of an entanglement bond, in which case it is attractive and repulsive at same time.

Instead, among charged particles, even though fundamental, with same sign (for example among free protons or among free electrons which are made to collide one another), the electrostatic repulsion force operates, as it is shown by the scattering experiments among alpha particles (nuclei of

${ }^{4} \mathrm{H}$ stripped of electrons, hence positively charged) and the target nucleus, it too endowed with a positive charge. In fact, these experiments allowed to deduce the nuclear radius from the angle of diffraction of the alpha particle beam resulting from the collision with the target nucleus, and this was possible by taking into account the Coulomb potential, and it means that between two nuclei, as well as in general among charged particles of same sign, does not operate the gravitoelectrostatic force, but the electrostatic 
one;

3) It acts, through an entanglement bond, between the real protons orbiting in the proton star, on the one hand, and the stars of the galaxy located beyond the innermost band of the space surrounding the black hole, on the other, in which case it is attractive-repulsive at same time;

4) It acts, through an entanglement bond, between the real protons orbiting in proton star, on the one hand, and the electrons that most likely revolve in the innermost band of the space surrounding the proton star, in which case it is attractive-repulsive at same time. After all in some recent researches, in particular see references [9] and [10], it has been shown that the possible trajectories of photons and of particles inside and around the black hole are compatible with a repulsive behavior of gravity. In the reference [11], then, a repulsive gravity was shown within the black hole even in the Stueckelberg Quantum formalism;

5) It acts, through an entanglement bond, between the shadow neutrons orbiting in neutron star, on the one hand, and the electrons that most likely revolve in the innermost band of the space surrounding the neutron star, on the other, in which case it is attractive-repulsive at same time;

6) It acts among the central shadow protons of proton star, in which case it is exclusively attractive;

7) It also acts in the self-orbiting phenomenon between the real particles, on the one hand, and the corresponding shadow particles, on the other, provided that the orbiting particles are the shadow ones (hence not also in the proton stars, where we have seen that the central shadow particles generate a strengthened gravitational attractive-repulsive field, not gravitoelectric), in which case it is attractive-repulsive at same time;

8) Unlike the gravitational force and the electrostatic one, the gravitoelectrostatic force, when it doesn't act in the self-orbiting phenomenon, is expressed through an entanglement relationship, implying the teleportation of the state of movement of the self-orbiting particles to the celestial bodies (i.e. the stars of the galaxy) or to the electrons which orbit around them, so that the orbit of the bodies or of the electrons will be 
as stable as possible if the potential gravitoelectric energy to which they are subjected is equal to twice their kinetic energy, but, due precisely to this entanglement bond, if the kinetic energy equals the potential energy, or even exceeds it, the balance doesn't break, provided that the orbiting celestial body or the orbiting electron move at the velocity given by the “entanglement velocity equation" - i.e. by (67) for the stars of galaxy, and by (75) for the electrons of atom - within the maximum distance predicted, only for the electrons of atoms, by (75-novies), and in the latter case the nucleus "tells" the electrons, which instantaneously receive the input, to emit photon in order to lose energy and to reach the innermost stability zone - within the range between the ideal radius given by (75octies) and that given by (82-ter) — where the gravitoelectric potential energy is greater than the kinetic one, thus restoring the dynamic balance between nucleus and electrons;

9) Since it depends on the product of the mass of the particles, not on their charge, it behaves more like the gravitational force rather than the electrostatic one, in the sense that, like the gravitational force, it can be exclusively attractive (for instance among the real nucleons of atom, or among the real neutrons of neutron star, or among the shadow central proton of proton star), or attractive and repulsive at same time (between nucleons and electrons, or in the self-orbiting systems), whereas the electrostatic force can be only attractive or only repulsive, but not attractive and repulsive at same time.

\section{- 5.16 - The Heisenberg's uncertainty principle: ontological characteristic of microscopic particles, or human limit?}

The principle states that it is not possible to predict the position and velocity of a microscopic particle.

Heisenberg initially formulated this principle in consideration of the extremely small size of the microscopic particles, whose position and velocity would have been altered by the photon beam necessary to observe them, so it was originary thought more in the sense of a human limit than in the sense of a natural 
law, but later was interpreted by quantum mechanics in a more rigorous sense, that is as an ontological characteristic of microscopic particles, so that it was improper to speak of position and velocity, by they having "dual" nature, i.e. both of particles and waves, which would have made the two parameters (speed and position) not applicable to the microscopic world.

Anyway, the present work has revealed the possibility of predicting with extreme precision both the velocity and the orbit of nucleons and of electrons, the latter to be presumed almost perfectly circular (better to say almost perfectly spherical, by the nucleon and the electron being not only a particle, but "also" a wave) if the potential strengthened-energy of nucleons (or of electrons) equals twice their kinetic energy, and with an elliptical shape increasingly eccentric (correctly saying, an ellipsoid, i.e. a sphere increasingly crushed at the poles, similar to a rugby ball) as the first energy approaches, reducing itself, the value of the second, which is why it seems appropriate to interpret Heisenberg's uncertainty principle in its originary less rigorous sense, that is, as a human limit affecting the observation activity, rather than as a basic principle of nature.

By the way, if the fundamental particles are "also" particles, in addition to being waves, nothing strange if they behave like all the particles, namely by always having a certain speed and position; otherwise these particles would be treated only as waves, which seems decidedly incongruous.

After all it has been recently and experimentally shown [12] that it's possible to predict the behavior of the atom, in particular it's possible to predict, even though only a short time before, the time after which the atom in excited state emits a photon, thanks to some specific signals detectable in the atom just before the quantum jump.

\section{- $\quad 5.17$ - Gravitational entanglement}

The results achieved here - which seem to lead with high probability in favor of the existence of an attractive-repulsive field even within the atom, even though characterized by the merger of the gravitational force with the electrostatic one, so as to be able to define it as an attractive-repulsive gravitoelectric forcesfield - argue in favor of the existence, in the macroscopic world, of a gravitational 
ligament between bodies endowed with mass very similar to the one existing between two microscopic particles, which allows us to bring the macroscopic gravitational phenomenon in the entanglement category.

The gravity force and the gravitoelectric one, indeed, according to the results achieved here, don't seem to be the consequence of any intermediation action, either by a field, as Einstein hypothesized, nor by the exotic particles exchanged between the bodies (the so-called "gravitons"), as quantum mechanics hypothesizes.

I realize that it is difficult to imagine gravitational interaction without the intermediation of anything, yet it is what it turns out from this work: it is an inexplicable action at a distance immediately operating, even though this is not entirely correct, since an ideal mediator would anyway exist, that is the gravitational (or gravitoelectric) ligament, but, as it has been said widely, it would operate instantly, contracting or dilating depending on whether it is attractive or repulsive, which would make the principle of locality, postulated by the theory of relativity with respect to gravitational interaction, inapplicable.

In the reference [16], by the way, it is reported a Newton's letter to Bentley in which the first said:

"Gravity must be caused by an agent [acting] consta[ntl]y according to certain laws, but whether this agent be material or immaterial is a question I have left to the consideration of my readers".

Therefore, the concept of an immaterial (or ideal) mediator ("agent"), as a cause of the force of gravity, is not rejected a priori by the great English scientist.

Although it is counterintuitive, we cannot help but notice that it is so, like it also happens in the phenomenon of quantum entanglement, where two particles (called "entangled", i.e. "intertwined" ", "linked" to each other) behave as if they were joined together, although they are physically miles away, in the sense that, if one acts on a particle, observing it, the other is immediately affected, always by having the opposite spin (rotation direction) and the same polarization with respect to the observed particle, and all this happens, I repeat, immediately, even if there is an infinite distance between the particles.

Force of gravity, even in its gravitoelectric variant, can be therefore 
considered a case of entanglement, in the sense that the bodies, all the bodies of the universe, provided that they have mass, are as related to each other, and if suddenly the mass of one varied, all other bodies would be immediately affected, even if placed at an infinite distance between them, exactly like it happens in the quantum entanglement, although in this last case the effect between the entangled particles is not attractive, but of different kind.

\section{- 6 - Conclusions}

In the present work, by moving from the assumption that the Sun (and all the massive bodies) produces, starting from a certain distance from it, attractive and repulsive gravitational forces at the same time, giving life to the movement of the planets according to the same principles of pendulum, I managed to derive a perihelion precession formula, a black hole radius formula and, above all, a formula of atomic nuclear radius, which was missing until now, all in excellent agreement with the observation and in a completely independent way of the Einstein's theory of relativity.

I have also shown that the nuclear radius formula can also be successfully used to predict the radius of neutron stars.

Furthermore I have found - always through the same principles that allowed me to achieve the above results, in particular through the modification of the Newtonian gravitational potential, in turn due to the different modus-operandi of gravity force - a formula of the non-decreasing orbital velocity of galactic stars, without considering dark matter.

From the mathematic results here obtained, I have demonstrate the existence of a gravitational strengthened-energy both in the perihelion precession phenomenon and in the black hole, that, in this latter case, prevents the bodies that enter the black hole, including the light, from coming out of it, as is shown by the fact that I have obtained the radius of the black hole from the perihelion precession angular velocity.

Then I have demonstrated the black hole is composed only of protons, and that it's similar to the nucleus of the atom and, analogously, the galaxy is similar to the atom, since the stars moving around the central nucleus in the same way as the 
electrons move around the atomic nucleus.

I have also found another similitude among atomic nucleus, black hole and neutron stars, namely the self-orbiting phenomenon existing in all the cases.

From the mathematical findings obtained in the present work it has also emerged the existence, both at the microscopic and the macroscopic level, of the gravitoelectric force (or, if one prefers, electro-gravitational force), resulting from the merging of the gravitational force with the electrostatic one, working exactly in accordance with Newtonian mechanics, although modified by the introduction of a repulsive force in addition and in opposition to the attractive one, that makes us understand the universe always works in the same way, both in macro and in micro.

It is also emerged the existence of the universal principle of specific asymmetry between gravitational potential energy and kinetic energy, as a cause of nuclear energy $E=M c^{2}$.

In this perspective, the present work can represent a potential unifying way between the macrocosm and microcosm mechanics.

\section{References}

[1] K. Heyde - Basic Ideas and concepts in nuclear physics - ISBN: 9780750309806.

[2] M. A. Preston and R. K. Bhaduri - Structure Of The Nucleus ISBN 13: 9780201627299.

[3] G.N. Afanas'ev et alt. - Effect of the imaginary part of the optical potential on the reaction cross sections - Physics of elementary particles and fields - BNL-TR-641.

[4] J.L. Basdevant, J. Rich, M. Spiro - Fundamentals in Nuclear Physics: from Nuclear structure to Cosmology — ISBN: 9780387250953.

[5] A. Proca, J. Phys. Radium 7, 347 (1936).

[6] Julian Heeck - How Stable is the Photon? - Phys. Rev. Lett. 111, 021801.

[7] S.K. Jain and Shailesh K. Jain - Conceptual Chemistry Volume I ISBN: 9788121927697. 
[8] Irina Dymnikova and Anna Poszwa - Classification and basic properties of circular orbits around regular black holes and solitons with the de Sitter center -2019 Class. Quantum Grav. 36105002.

[9] Sirachak Panpanich et al. — Particle motions and gravitational lensing in de Rham-Gabadadze-Tolley massive gravity theory - Phys. Rev. D 100, 044031.

[10] Keisuke Nakashi et al. - Null geodesics and repulsive behavior of gravity in $(2+1) D$ massive gravity - Progress of Theoretical and Experimental Physics, Volume 2019, Issue 7, July 2019, 073 E02 https://doi.org/10.1093/ptep/ptz062

[11] D. M. Ludwin1 and L. P. Horwitz - Gravitational repulsion within a black hole using the Stueckelberg quantum formalism - Journal of Mathematical Physics 52, 012303 (2011) https://doi.org/10.1063/1.3533399

[12] Z. K. Minev et al. - To catch and reverse a quantum jump mid-flight - Nature volume 570, pages 200-204 (2019) https://doi.org/10.1038/s41586-019-1287-Z

[13] G. Michaud, L. Scherk, and E. Vogt - Nuclear Optical Model and Wave Properties: Barrier Penetration, Reflection, Absorption, and Resonance - Phys. Rev. C 1, 864 - Published 1 March 1970.

[14] C.A. Bertulani - Nuclear reactions - Wiley Encyclopedia of Physics, ISBN-13: 978-3-527-40691-3 - Wiley-VCH, Berlin, 2009 https://arxiv.org/abs/0908.3275

[15] Bogdan Povh, Klaus Rith, Christoph Scholz, Frank Zetsche, Martin Lavelle — Particles and Nuclei - ISBN: 978-3-662-46321-5 - DOI: https://doi.org/10.1007/978-3-662-46321-5

[16] Berkovitz, Joseph (2008). "Action at a Distance in Quantum Mechanics". In Edward N. Zalta (ed.). The Stanford Encyclopedia of Philosophy (Winter 2008 ed.).

[17] S. Wycech et al., Antiprotonic studies of nuclear neutron halos, Phys. Rev. C 54, 1832 - Published 1 October 1996 - DOI: https://doi.org/10.1103/PhysRevC.54.1832 
[18] Wycech S., Smolańczuk R. (1995) - Nuclear Neutron Haloes as Seen by Antiprotons. In: Adam J., Dobeš J., Mach R., Sotona M., Dolejší J. (eds) Mesons and Light Nuclei '95. Few-Body Systems, vol 9. Springer, Vienna, DOI: https://doi.org/10.1007/978-3-7091-9453-9_12

[19] Schmidt, A., Pybus, J., Weiss, R. et al. Probing the core of the strong nuclear interaction. Nature 578, 540-544 (2020). https://doi.org/10.1038/s41586-020-2021-6

[20] Napolitano, R.. The gravitoelectric nuclear energy. Preprint at: https://doi.org/10.31219/osf.io/68fbs (2020, July 25) 